University of Rhode Island

DigitalCommons@URI

Open Access Dissertations

2017

\title{
Three Essays on the Impact of Information on Nonpoint Source Polluters' Behavior
}

Haoran Miao

University of Rhode Island, haoran_miao@uri.edu

Follow this and additional works at: https://digitalcommons.uri.edu/oa_diss

\section{Recommended Citation}

Miao, Haoran, "Three Essays on the Impact of Information on Nonpoint Source Polluters' Behavior" (2017). Open Access Dissertations. Paper 668.

https://digitalcommons.uri.edu/oa_diss/668

This Dissertation is brought to you for free and open access by DigitalCommons@URI. It has been accepted for inclusion in Open Access Dissertations by an authorized administrator of DigitalCommons@URI. For more information, please contact digitalcommons-group@uri.edu. 
THREE ESSAYS ON THE IMPACT OF INFORMATION

ON NONPOINT SOURCE POLLUTERS’ BEHAVIOR

BY

HAORAN MIAO

A DISSERTATION SUBMITTED IN PARTIAL FULFILLMENT OF THE REQUIREMENTS FOR THE DEGREE OF

DOCTOR OF PHILOSOPHY

IN

ENVIRONMENTAL AND NATURAL RESOURCE ECONOMICS

UNIVERSITY OF RHODE ISLAND 


\section{DOCTOR OF PHILOSOPHY DISSERTATION}

OF

HAORAN MIAO

APPROVED:

Dissertation Committee:

$\begin{array}{ll}\text { Major Professor } & \text { Emi Uchida } \\ \text { Todd Guilfoos } & \text { Arthur J. Gold } \\ \text { Nasser H. Zawia } \\ \text { DEAN OF THE GRADUATE SCHOOL }\end{array}$

UNIVERSITY OF RHODE ISLAND

2017 


\begin{abstract}
Nonpoint source pollution is recognized as the primary cause of water pollution in the United States and has many adverse environmental effects in other areas such as Europe and China. In this dissertation research, I examine the role of information in managing nonpoint source pollution through voluntary programs and regulatory policies. Specifically, I look into the effect of informational nudges, information appealing to people to act, and financial incentives to reduce nonpoint source pollution through behavioral changes. Also, I investigate the impact of information on nonpoint source polluters' behavior under the ambient-based policy when the environmental uncertainty exists at the individual level, and the information about other polluters' action vary. We use three methods to study the impact of information on nonpoint source polluters' behavior: a randomized field experiment, a controlled laboratory experiment, and one integrated agent-based model. We test the following general hypotheses: (1) Informational nudges affect nonpoint source polluters' behavior, but the effect is not persistent when we combine informational nudges with financial incentives to affect behavior. (2) Decreased environmental uncertainty leads to more efficient allocation of abatement efforts across nonpoint source polluters and better social efficiency under the ambient-based policy. (3) Under the ambient-based policy, different levels of environmental uncertainty and the ability to obtain information about other polluters' actions affect nonpoint source polluters' learning pattern and equilibrium behavior.

I find that informational nudges and financial incentives both work to change behavior, but they may substitute each other, especially when the financial incentive is
\end{abstract}


small. The ambient-based policy is effective when uncertainty levels vary, but eliminating environmental uncertainty leads to less pollution. Different levels of environmental uncertainty and information disclosure induce different learning patterns of nonpoint source polluters. The agent-based model shows that high degrees of uncertainty lead to behavioral differences in the long run given the agents can observe other group members' behavior. 


\section{ACKNOWLEDGMENT}

I give my gratitude to my advisor, Dr. Emi Uchida. During my five-and-half-year graduate studies, she inspired me in her classes to develop my research interests, supported me when I have much confusion in my research path, encouraged me to take the first step to accomplish many academic necessities, and help me patiently to develop and complete this dissertation. She is an informative advisor and a great mentor to me.

Also, I want to thank my committee members, Dr. Todd Guilfoos, Dr. Arthour J. Gold, Dr. Thomas W. Sproul and Dr. Gavino Puggioni, and coauthors, Dr. Simona Trndafir and Dr. Michel Prirce for their valuable suggestions in this dissertation. I was less confused by known unknowns after numerous meetings with you. I also give my sincere thanks to Dr. Tracey Dalton for being my defense chair.

Lastly, I thank my wife, my daughter, my parents, my parents-in-law, my brother and sister. Your love supports me to go through the lengthy and continuing academic journey. 


\section{PREFACE}

I use the manuscript format for this dissertation which consists of three manuscripts and appendices. The composition of three manuscripts follows the requirement of a specific journal. The goal of this thesis is to understand the role of information in managing nonpoint source pollution through voluntary programs and regulatory policies.

The first manuscript report results of a randomized field experiment which we use to investigate the performances of informational nudges and financial incentives to change homeowners' behavior when they manage their lawns.

The second manuscript uses a laboratory experiment to examine nonpoint source polluters' behavior under the ambient-based policies when the environmental uncertainty exists at the individual level, and the information sets provided to the experimental subjects vary.

The third manuscript looks into nonpoint source polluters' learning patterns using an experience weighted attraction learning model. We build up an agent-based model in which we calibrate the agents with parameters from the learning model to scale up the findings from the experiment in the second manuscripts.

A conclusion chapter follows the third manuscript. It summarizes all the three manuscripts, policy implications of this dissertation study, and point out directions for future research.

The appendices include a survey and other materials we used to collect data for the first manuscript and an experimental instruction for conducting the experiment in the second and third manuscripts. 


\section{TABLE OF CONTENTS}

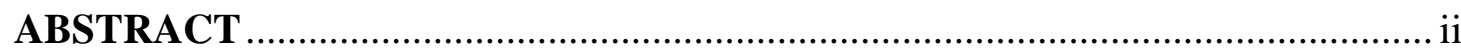

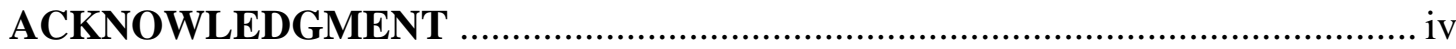

PREFACE

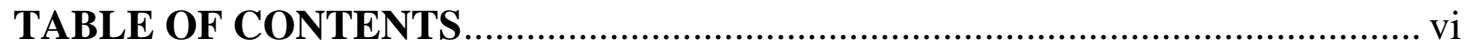

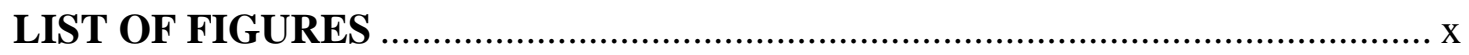

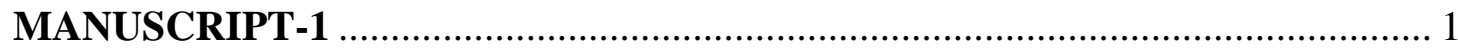

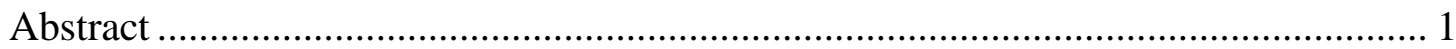

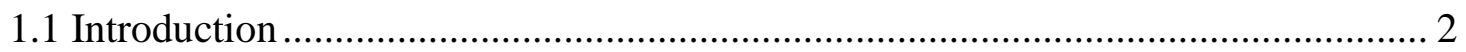

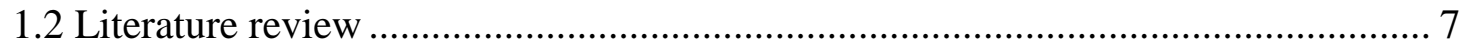

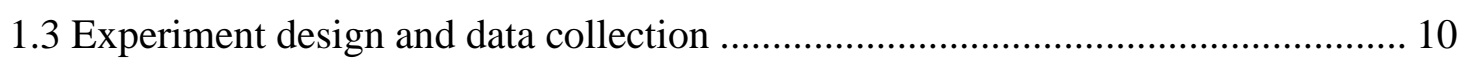

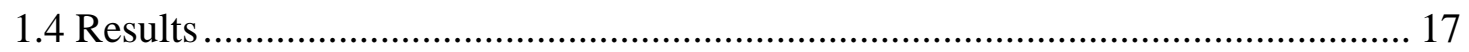

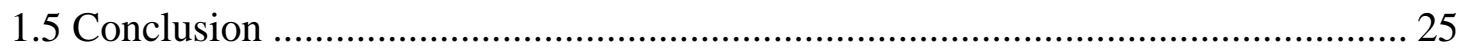

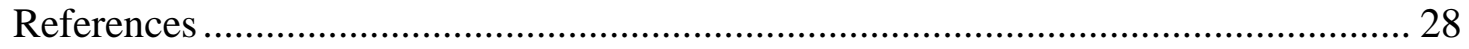

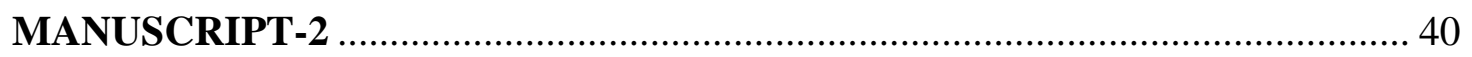

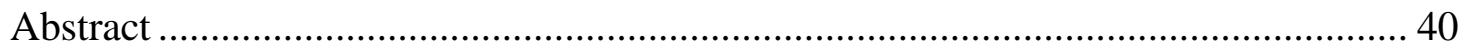

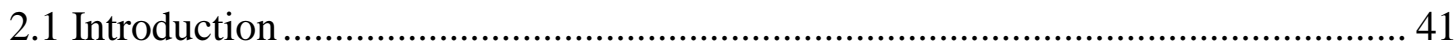

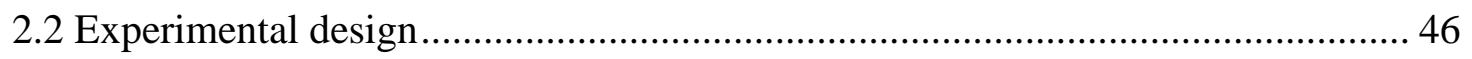

2.3 Theoretical predictions and hypotheses …………............................................ 54

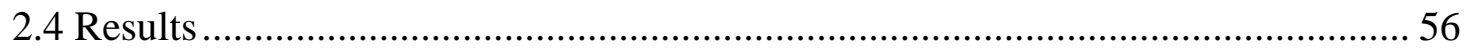

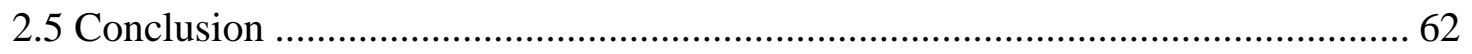

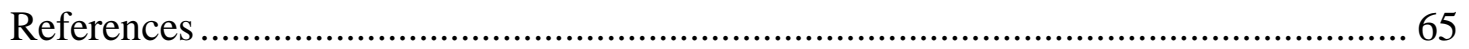


MANUSCRIPT-3

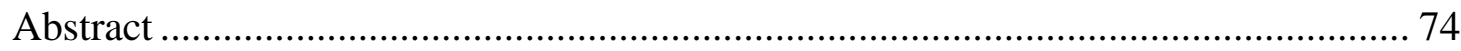

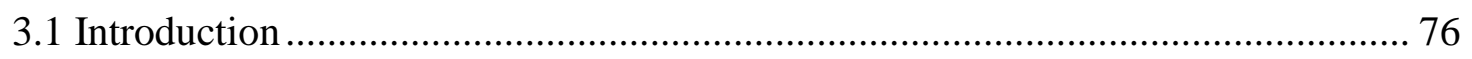

3.2 Experimental design, Parameterization, and Protocol ...................................... 81

3.3 The learning model and results of econometric analysis ................................. 84

3.4 ABM framework, calibration, and simulation results ................................... 90

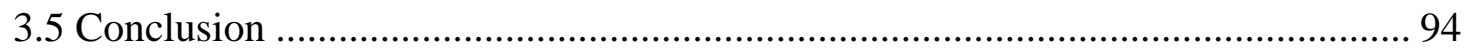

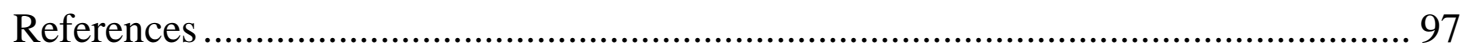

POLICY IMPLICATION AND FUTURE DIRECTIONS FOR RESEARCH........ 107

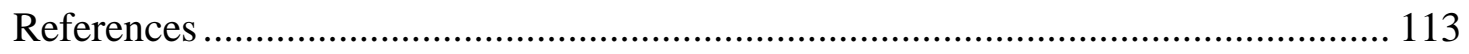

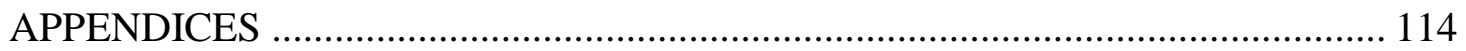

Appendix I: Field experiment survey......................................................... 114

Appendix II: A list of organizations who distributed our survey.......................... 203

Appendix III: An example of recruitment advertisement ................................... 204

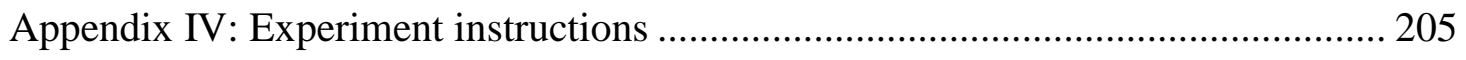




\section{LIST OF TABLES}

TABLE

PAGE

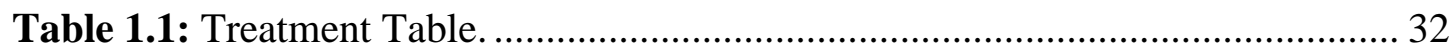

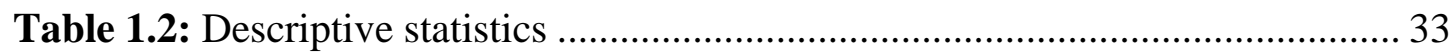

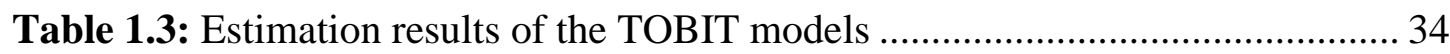

Table 1.4a: Estimation results of the OLOGIT models ........................................... 35

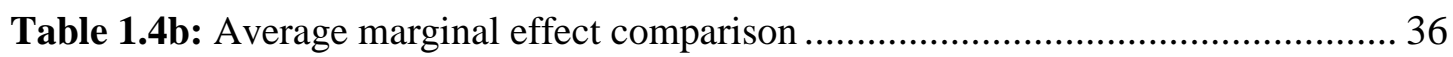

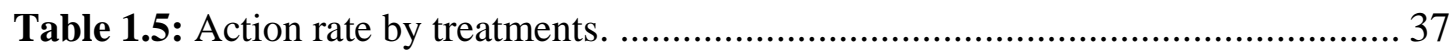

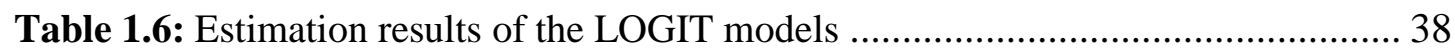

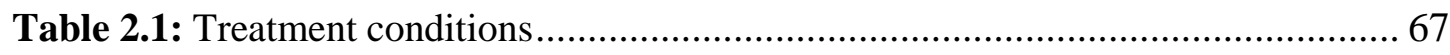

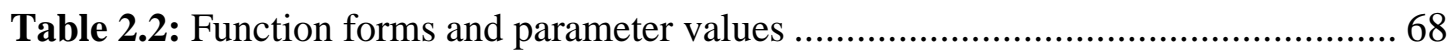

Table 2.3: Profit-maximizing values of production and abatement for (expected) emission

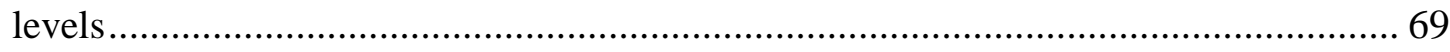

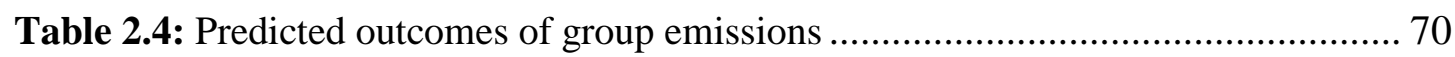

Table 2.5: Average expected efficiency of treatments. ............................................ 71

Table 2.6: Effects of uncertainty level and information on efficiency ........................ 72

Table 2.7: Marginal effects of uncertainty and information disclosure based on the

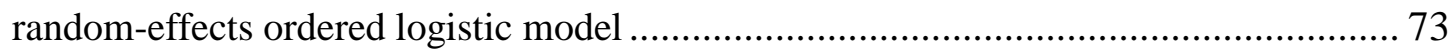

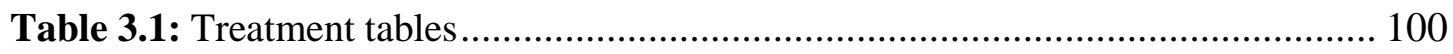

Table 3.2: Function forms and parametrization ............................................. 101

Table 3.3: Profit-maximizing values of production and abatement .......................... 102

Table 3.4a: Parameter estimates of the EWA learning model for no-information

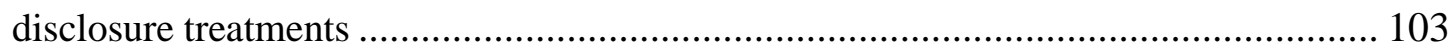


Table 3.4b: parameter estimates of the EWA learning model for information disclosure

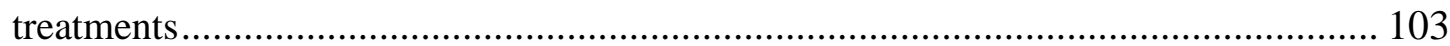




\section{LIST OF FIGURES}

FIGURE

PAGE

Figure 1.1: Indirect effects of the financial incentives through NEP Scale................... 39

Figure 3.1a: Average social efficiency over time (No information disclosure)........... 104

Figure 3.1b: Average social efficiency over time (With information disclosure)......... 104

Figure 3.2a: Average individual emission over time (no information disclosure) ....... 105

Figure 3.2b: Average individual emission over time (with information disclosure) ... 105

Figure 3.3a: Simulated average individual emission over time (no information disclosure) 106

Figure 3.3a: Simulated average individual emission over time (no information disclosure) 106 


\title{
MANUSCRIPT-1
}

The effect of informational nudges and financial incentives to reduce nonpoint source pollution: A randomized controlled trial

To be Submitted to Environmental and Resource Economics, under first round of revision

\author{
by \\ Haoran Miao ${ }^{a}$, Simona Trandafir ${ }^{a}$, Emi Uchida ${ }^{a}$, and Michael Price ${ }^{b}$ \\ ${ }^{a}$ Department of Environmental and Natural Resource Economics, University of Rhode Island, Kinston, RI \\ ${ }^{b}$ University of Alabama, Tuscaloosa, $A L$
}

\begin{abstract}
Policymakers often use voluntary programs to control nonpoint source pollution from residential lawns. We run a field experiment to test whether informational nudges and financial incentives can motivate residents to choose green-certified lawn care services voluntarily. We find that informational nudges spur voluntary behavior conditionally. However, financial incentives, especially a small one, to encourage behavior do not reinforce the effect of the informational nudges. Our evidence shows that the informational nudges and the financial incentives are substitutes. These findings are potentially important for state and federal agencies as well as advocacy groups interested in promoting best management practices.
\end{abstract}

JEL Classification: $C 93, Q 25, Q 53, D 03$

Keywords: Nonpoint source pollution, voluntary program, informational nudges, financial incentives, and field experiment 


\subsection{Introduction}

Nonpoint source (NPS) pollution is recognized as the primary cause of water pollution in the United States [EPA, 2007] and has many adverse environmental effects in other areas such as Europe [EEA, 2007] and China [Sun et al., 2012]. It is difficult to control NPS pollution due to its diffuse nature and resulting information asymmetries between an environmental regulator and the agents who contribute to the NPS pollution problem [Xepapadeas, 2011]. To internalize external environmental damages caused by NPS pollution and circumvent the information problems, there are standard regulatory policy instruments such as input tax [Griffin and Bromley, 1982], ambient-based instrument [Segerson, 1988] and random punishment [Alpízar et al., 2004]. Theoretically, they are promising to alter behavior that would inhibit good water quality. However little progress on NPS pollution regulation has been made, at least in the United States, because of various barriers, including significant political barriers [Craig and Roberts, 2015], few experimental designs and heterogeneity of spatial and temporal scales for behaviors and measured outcomes [Rissman and Carpenter, 2015].

Currently, local, state, and federal initiatives in the United States mainly take the voluntary approach to stimulate behavior change to reduce NPS pollution. For example, farmers in the agricultural area are encouraged to adopt pollution control through local, state and federal financial supports [Shortle et al., 2012]. Residents in the urban and suburban area often encounter education campaigns which nudge people to take voluntary behavior to reduce polluted runoff. Appeals such as "water and fertilizer your lawn properly," "leave clips on your lawn" and "use a rain barrel" frequently appear in those programs [e.g., Eisenhauer et al., 2010; Bakacs et al., 2013]. From a broader point of view, 
policymakers all over the world widely favor the use of voluntary approaches as a way to improve environmental qualities [Segerson, 2013]. Though treated as second-best instruments, voluntary approaches can be cost-effective in encouraging conservation and environment protection with thoughtful designs. Successful examples of those designs include payments for ecosystem services schemes under which landowners are paid for implementing conservation practices [e.g., Ferraro and Kiss, 2002], or unilateral initiatives under which polluters take voluntary actions to reduce pollution [e.g., Ahmed and Segerson 2011].

Inspired by these voluntary programs, we conduct a randomized controlled field experiment to examine whether on so-called "green nudges" [Schubert, 2017], can promote voluntary behavior to reduce NPS pollution from residential lawns. We also test whether informational nudges spur subjects' non-pecuniary motivation such as moral costs [Levitt and List, 2007] in NPS pollution settings and lead to an increase in demand for green services. To do so, we impose a cross-randomized treatment in which respondents receive financial incentives to cover a portion of the upfront cost of buying green services. We postulate that in the field experiment financial incentives can also stimulate the demand for green services as the informational nudges can, but it may crowd out the demand nudged by the information.

Although there are many sources of NPS pollution, household lawn fertilization represents one of the causes of water quality degradation in many regions [EPA, 2005]. According to Milesi et al. [2005], co-authored by NASA scientists, lawns now cover an area larger than any irrigated agricultural crop in the U.S. As the runoff moves away from the residential area, it carries pollutants, such as excessive nutrients and pesticides from 
lawns, and deposits them into receiving waterbodies. Household lawn fertilization is significant nitrogen inputs in the urban waterways [Hobbie et al., 2016]. With proper lawn care, residents can reduce the negative impact of runoff from lawns, however [Spence et al., 2013]. State and local agencies in the United States have mostly relied on education programs and extension campaigns to send information to homeowners and nudge them to voluntarily adopt best management practices (BMPs) on private lawns [e.g., Dietz, 2004; Kelly et al., 2012; Bakacs et al., 2013]. Although understanding the effectiveness of these education and extension programs on the adoption of BMPs is important to policymakers, one difficulty in measuring the outcomes of those programs is the researchers' inability to observe the subjects' lawn care behavior, such as mowing the lawns high or reducing fertilizer use. Thus, the leading metrics to measure the success of these programs have been based on households' stated preferences in interviews or surveys towards BMP adoption [e.g., Dietz, 2004; Ballentine, 2005].

In this study, we advance the literature by examining how such information can nudge homeowners to choose lawn care companies that provide lawn care BMPs in the field experiment. We develop the experiment around a new certification program by the Rhode Island Department of Environmental Management (RIDEM) which green-certifies the lawn care businesses that commit to a list of BMPs. Specifically, we test how information nudges affect homeowners' decisions to adopt green-certified lawn care contracts. We also give real financial incentives randomly to a portion of homeowners by offering rebates for green-certified lawn care contracts to check the interaction effects of financial incentives and information nudges on homeowners' choices of green-certified lawn care contracts. 
We partnered with the local green-certified lawn care industry to run the experiment and recruited residents in the area serviced by the green-certified companies as research subjects. From the experiment, we obtain three indicators to measure the impact of informational nudges and financial incentives on promoting voluntary behavior: (i) homeowners' stated WTP for green-certified lawn contracts; (ii) reported willingness to hire green-certified lawn companies based on a Likert scale; and (iii) information acquisition requirements which mean that the respondents leave their contact details (their names, email addresses, and phone numbers) to the green-certified lawn care companies to get contacted, including free estimates. Ideally, we would use the uptake of contracts as the outcome of interest. However, we were not able to collect sufficient data to test the effects on contract uptake. We examine the three indicators above as alternative outcomes. ${ }^{1}$ However, we claim that the third indicator of whether or not the subject left their contact information is a significant departure from the existing literature because leaving private information can be considered a costly action.

We hypothesize that the informational nudges add a premium to the WTP for the green-certified lawn care contracts, increase the stated likelihood to hire a green-certified lawn care company and motivate more people to take the first step towards an environmentally friendly lawn care practice. Financial incentives would have a similar effect to the informational nudges on the reported probability to hire and motivating people to take action. However, when we implement them together, there may be the crowd-out

\footnotetext{
${ }^{1}$ We have several conjectures why the uptake was small. First, the companies had limited resources to reach out to the subjects who were interested in the green-certified contracts. For subjects who provided contact information for the lawn care companies, we passed on the information to the three companies and they were then responsible for the negotiation with the homeowners, but a substantial portion of the homeowners were never reached. Second, we did not conceal the information who got the financial incentives to the lawn care companies. They gave quotes to homeowners strategically. For example, they priced their services higher given the potential customers received financial incentives.
} 
effect. To the best of our knowledge, our study is the first to use a field experiment to evaluate the effect of informational nudges using both stated preferences and revealed preferences together at the specific settings.

We find that in our study, on average, the respondents in the informational nudge treatment group state a significantly larger WTP for green-certified lawn care contracts, but this premium nudging by the information diminishes as the cost of the lawn care increases. When we restrict the samples to those who report that they are likely to contract with green-certified lawn care companies, the nudging premium is still positive but not statistically significant; the decreasing trend over the cost still exists but not statistically significant either.

We also find that the informational nudges and a high financial incentive significantly increase the reported likelihood to seek green-certified lawn contracts. However, a low financial incentive alone seems to have limited effect on the respondents' Likert Scales of hiring a green certified lawn care company and adding financial incentives over does not statistically significantly increase the stated likelihood after we show the informational nudges to the respondents.

The informational nudges have a significant effect on nudging people to take the first step to ask for free estimations from the green-certified lawn care companies. Financial incentives are also great instruments to nudge behavior, but we find evidence that shows financial incentives and the informational nudges substitute each other. Only a high financial incentive can induce a significantly greater treatment effect compared to the nudge-only instrument. 
We organize the article as follows: Section 2 goes through some related literature. Section 3 presents the experiment design and the data collection process. Section 4 formulates the detailed results. Section 5 states our conclusions and recommendations.

\subsection{Literature review}

Nudge theory is a concept in behavioral science and economics which has been widely applied since the seminal book "Nudge: Improving decisions about health, wealth, and happiness" by Richard H. Thaler and Cass R. Sunstein [2008]. They define nudges as "any aspect of the choice architecture that alters people's behavior in a predictable way without forbidding any options or significantly changing their economic incentives.". Schubert [2017] raises an idea about "green nudges" and considers it as "nudges that aim at promoting environmentally benign behavior.". However, since the exact definition of nudges is somewhat controversial and there is no strict line between nudges and incentives [Hansen, 2016], we use "informational nudges" and "financial incentives" to differentiate the two kinds of treatments in our study. The first treatment offers information, while the second treatment provides financial incentives to homeowners to nudge the purchasing behavior of green-certified lawn contracts.

Information can affect people's wiliness to pay for market goods. Depositario et al. [2009] find that WTP bids for golden rice are higher under positive information than no information, negative information and two-sided information scenario. Rousseau and Vranken [2011] demonstrate that the provision of information on the actual environmental and health effects of organic apple production increases consumers' price premium. One study by Aldrovandi et al. [2015] shows that exposing people to two different types of 
information about how their consumption is ranking creates an over 30\% WTP gap for healthy food. Consumers even react to the information provided by eco-labeling. For example, Stemle et al. [2016] find that Marine Stewardship Council certification significantly improves ex-vessel prices of some fishes.

Information can also nudge consumers' behavior change. For example, according to the study by Mathios [2000], people significantly consume fewer salad dressings with very high-fat levels after producers were mandatorily required to disclose nutritional content by the Nutrition Labeling and Education Act. Bertrand \& Morse [2011] show that information showing the adding-up effect of loan fees over several pay cycles helps people overcome cognitive bias and results in $11 \%$ less borrowing in the subsequent four months. There are also a handful of field experiments conducted to test the impact of informational nudges on quantity and quality of people's energy consumption. For instance, Allott [2011] reports that, on average, consumers reduce $2 \%$ energy consumption when nudged by the Home Energy Report letters which display the comparison of their electricity use to that of their neighbors.

Nevertheless, the effect of informational nudges on behavior is highly contextdependent, and the effect of different types of information varies. Fellner et al. [2011] run a field experiment in Austria to test the effectiveness of various normative messages sent to potential evaders of TV license fees. While the "threat" treatment which makes a high detection risk salient is useful, neither appealing to morals nor imparting information about others' behavior induces significant behavior change. Costa \& Kahn [2013] find that the informational nudges could have an adverse effect for some political conservatives who 
may increase their electricity consumption only because they resent the reports that link their behavior to that of their neighbors'.

When we couple the informational nudges with the financial incentives, the effect is even more complicated. The tension between intrinsic motivation (which can derive from informational nudges) and extrinsic incentives (which might be offered by external agents) is well known among the growing bodies of field experiment literature [e.g., Alpízar and martinsson, 2011; Lacetera et al., 2012;]. The recent research by Pellerano et al., [2016] uses both intrinsic motivation and extrinsic motivation to induce consumers' energy conservation, and they detect a backfire of extrinsic motivation in their experiment. Extrinsic motivation may have a crowding-out effect especially when incentives are small. However, as Gneezy et al. [2011] argue a large incentive may have a stronger price effect than the crowding-out effect so that it can promote conservation in the short run. Our study serves as field evidence of this argument in NPS pollution voluntary programs settings, and our experiment design can also test for the potential crowding-out effect of financial incentives.

Many differences exist between lawn care contracts and standard market goods such as healthy food, electricity or drinking water even there are markets for lawn care contracts. We do believe those differences affect the outcomes of the survey. For example, households have different sizes of lawns which may result in very different stated WTP for green-certified lawn care; or some households might have good relationships with lawn care companies they previously contracted with which may affect their decision to switch to a green-certified one. Thus, we also record lawn care practices and individual characteristics in the experiment. By controlling those components as other literature does, 
we expect to improve the precision of the treatment effect estimation. Furthermore, we collect two pieces of unique personal information from the respondents echoing the literature that shows the New Ecological Paradigm (NEP) Scale and the Self-Report Altruism (SRA) Scale are good predictors of pro-social behavior. The NEP Scale is an estimate of endorsement of pro-environmental attitudes [Dunlap et al., 2000] and the SRA Scale is a measure of altruistic personality [Rushton et al., 1981]. In the online survey conducted by Attari et al. [2016], for example, people's pro-social actions have a strong correlative relationship with the NEP Scale. The research by Otto and Bolle [2011] shows that the SRA Scale is related to the charity giving, but not to the blood donation behavior.

\subsection{Experiment design and data collection}

\subsubsection{Experiment design}

We conduct the research in Rhode Island and neighboring counties in southern Massachusetts and western Connecticut. This area is one of the most densely populated in the United States and has high fractional turfgrass coverage [Milesi et al., 2015]. The urbanization process is still active. When land is transformed for urban and suburban usage, a lawn is often planted as ground cover on open spaces attached to houses and buildings [Jenkins, 2015]. The importance of neighborhood appearance and concern for aesthetics drive residents to water and fertilize lawns frequently and use other practices to care for their lawns [Nielson and Smith, 2005]. Significant NPS pollution is generated from those lawn care practices, and it is recognized as one of the causes of water quality impairments in some area [e.g., RIDEM, 2015]. 
In 2015, RIDEM started the program to green-certify lawn care companies who adopt BMPs to reduce their environmental impact when managing residential lawns. The green-certified lawn care companies fulfill a menu of BMPs covering a range of activities focusing on efficient turf management and water conservation. They protect environmental quality by reducing the source of pollution, conserve water and other natural resources and contribute to the sustainability of lawn care. Green-certified lawn care companies need to adopt additional best management practices to get re-certified every two years. Although it is the smallest state in the United States, Rhode Island has about 400 lawn care businesses according to RIDEM. Only ten lawn care companies (about 2.5\%) were green-certified as of January 2017.

The green certification program by RIDEM is a good example of the state-level effort to reduce NPS pollution by promoting voluntary "green services" from the supply side. Lawn care companies' behavior varies largely when managing residential lawns [Law et al., 2004]. According to meeting memos with lawn care professionals in Rhode Island, those who pursue this green-certification want to fulfill social responsibility and also hope that the green-certification will bring customers for their business. Apparently, if more customers adopt lawn care contracts because of the green certification, more lawn care companies will react to the signal and minimize their environmental impact. As a side goal, we conduct the experiment to find good ways to endorse and publicize green-certification.

We partnered with a state-wide professional landscape association [the Rhode Island Nursery and Landscape Association (RINLA)] and three green-certified landscaping companies to run the experiment. In contrast to measuring homeowners' direct supply of BMPs as outcomes in regular voluntary programs to control NPS pollution, we estimate 
their indirect supply through contracting with green-certified lawn care companies in this study which is more observable.

We test the effect of two general treatments in the experiment to promote the greencertified lawn care contracts: informational nudges and financial incentives. The test treatments are varied between participants in a general two-by-two design (see Table 1.1, column 1, 2 and 3) and implemented in a structured survey coded in Qualtrics, an online survey software. Respondents of the survey are all exposed to the introduction of greencertified lawn care companies, and two-thirds of them are randomly chosen to receive the informational nudges treatment. We introduce a cross-randomized incentive treatment after the first randomization of information. Three-quarters of respondents receive financial incentives randomly, and one-quarter of them receive none. To differentiate the effect of different informational nudges and different level of financial incentives, we implement two types of nudges and two levels of incentives in the experiment (see Table 1.1, column 4, 5 and 6 for more details).

The subjects are first introduced to RIDEM's Green Certification Program and the green-certified lawn care companies (page 130, Appendix I). The first segment introduces the history of the green certification program and some BMPs conducted by lawn care companies using bullet points. The second segment reinforces the contents of the bullet points using an infographic developed for this experiment by a professional designer. The third segment attaches a few reputable sources about BMPs for lawn care practices from three state universities (the University of Massachusetts, the University of Connecticut and the University of Rhode Island). Based on results of the report about lawn care behavior [Eisenhauer et al., 2010], we design another infographic (Nudge 1) that contains the salient 
information about the relationship between lawn care practices and water quality. We vision that salient information would increase homeowners' moral cost and thus change their behavior. We display Nudge 1 to all the respondents who are in the general informational nudges treatment group. The information such as "Runoff flows into aquatic habitats, where, just like your lawn, fertilizer makes plants grow. However, too much growth can lead to not enough oxygen in the water, killing fish and other aquatic lives." is shown in the infographic of Nudge 1. We also design another infographic (Nudge 2) that displays the information about how many the respondents' neighbors choose to have greencertified lawn care contracts in 2016 based on the statistics we obtain from RINLA. We hypothesize that social comparison can also change homeowners' behavior. Half of the respondents in the general informational nudges treatment group are exposed to Nudge 2 randomly. (See the survey in Appendix I for more details about the introduction of the certification programs and green-certified lawn care companies and infographics for informational nudges.)

Two levels of financial incentives are designed and distributed to the respondents randomly. A low financial incentive covers twenty-five percent of a typical green-certified lawn care contract of which the average value is $\$ 300$ according to RINLA, while a high financial incentive covers half of the contract. Due to the limited budget, we cap the low financial incentive to $\$ 75$ and the high financial incentive to $\$ 150$. Two-thirds of the respondents who are in the general financial incentive group receive the low financial incentive randomly, and one-third of them are randomly exposed to the high financial incentive. 


\subsubsection{Survey Design and data collection}

The survey contains five parts. The first part of the survey includes screening questions which ensure the respondents are eligible. An eligible respondent must have his or her house properties in Rhode Island and some areas in Massachusetts and Connecticut. We include areas in the other two states because Rhode Island has small geographical area and many lawn care companies have potential customers there. Also, the respondent must be responsible for making decisions on lawn maintenance. We also exclude those households that hired green-certified lawn care companies in 2016 from our survey. The second part begins with a consent form. In the consent form, we state the affiliation and the purpose of the survey. We include a short description and expected time that the respondents may take to finish all the questions. We embed some clarification requested by the Institutional Review Board (IRB) at the University of Rhode Island in the consent form. If the respondents agree all the statements in the consent form, they may continue to answer the survey. The rest of part two asks the respondents questions about lawn characteristics and lawn management practices in 2016.

We display the information about green-certified lawn care companies and the informational nudges in the third part of the survey. One-third of the respondents receive Nudge 1 randomly, and another third receive Nudge 1 and Nudge 2 together. We ask the WTP for green-certified lawn care contracts after the nudge randomization is over. The fourth part of the survey is the randomization of giving away incentives. The Likert scale question about their willingness to obtain services from one green-certified lawn care companies is asked based on different combination of the informational nudges and financial incentives. The Likert scale has six levels for this question, from "extremely 
unlikely" to "extremely likely". At the end of the fourth part, we ask the respondents whether or not they are interested in receiving more information from the three greencertified lawn care companies that collaborate with us. If they are interested, we then ask the respondents for permission to send their contact details to the three green-certified lawn care professionals. Demographic questions including gender, age, education, and income and the question matrices to tease out the respondents' NEP Scale and SRA Scale comprise the fifth part of the survey.

The NEP Scale is designed to gauge the environmental concern of people using a survey instrument constructed of fifteen statements. The survey instrument includes the statement such as "we are approaching the limit of the number of people the earth can support." or the statement "if things continue on their present course, we will soon experience a major ecological catastrophe.". We ask the respondents whether they agree or disagree those statements, and they answer the questions by choosing from seven levels from "completely disagree" to "completely agree". We assign a value of zero to "completely disagree" and a value of six to "completely agree". The NEP Scale equals the average of the values of the fifteen statements.

The SRA Scale is designed to measure people's altruistic tendency by letting people self-report the frequency with which they engage in 20 altruistic acts primarily toward strangers. Respondents report the frequencies of the acts on a five-point scale ranging from "never" to "very often". A value of zero is assigned to "never" and a value of four for "very often". The SRA Scale equals the summation of the values of the 20 acts.

We conducted four sessions of focus groups to make sure the respondents understand our survey questions. Group participants were recruited from Craigslist and 
compensated with $\$ 40$ for their time. Eligible participants were those who were over 18 years of age, had a lawn and were responsible for its maintenance. We conducted all focus groups at the Robert L. Carothers Library of the University of Rhode Island. During the focus groups, the participants were asked to take the survey first and then provide us with their feedback. On average, the participants spent 19 minutes to finish the survey. Questions such as "Are there any questions that are not clear?" or "What is the message of this infographic?" were asked in the guided feedback session following the completion of the survey. Participants 'comments and suggestions were then used to improve the survey, whenever appropriate. We also sent the survey to outreach personnel at University of Rhode Island and lawn care professionals for further opinions. The survey was officially distributed to the respondents at the end of February 2017 and was supposed to conclude by the end of March 2017. However, due to inclement weather and the subsequent delay of lawn care season, we extended the survey closing date until April $15^{\text {th }}$.

The survey was coded in Qualtrics and distributed electronically. The survey respondents were recruited through two channels: Qualtrics panel and local organizations' email listservs ${ }^{2}$. Qualtrics use by the invitation-only online panel recruitment methods to avoid self-selection and professional survey takers. Thus many researchers utilize it to recruit subjects for their studies [Brandon et al., 2013]. Our survey was also distributed to the respondents by local schools, environmental organizations and local newspapers. We reached out to school district superintendents, school principals, directors of environmental organizations and newspapers and representatives of city governments to communicate the availability to distribute the survey to their email listservs. Five schools, twelve

\footnotetext{
${ }^{2}$ Our study did not reach the sample size needed based on power calculation, however.
} 
environmental organizations, one local online newspaper and one city hall, agreed and managed to post our survey on their newsletters sending to their audiences routinely. The research team created advertisements for recruiting respondents and made minor changes according to volunteer organizations' requests. We offered a \$2 Amazon gift card to any respondent who finished our survey as a small thank-you gift. The respondents could obtain the gift cards by signing their names and leaving email addresses. They could also choose to donate the gift cards to the organization that sent them our survey. We attach a full list of those organizations in Appendix II. We also include one example advertisement in Appendix III. ${ }^{3}$

Survey data were downloaded from Qualtrics and updated in our internal database twice per week (Monday and Thursday) during the survey period. The research team routinely checked the data, sent out the Amazon gift cards and forwarded interested respondents' information to lawn care companies. We collected 1,736 answered surveys in total, 1,000 of these from the Qualtrics panel and 736 from schools and local organizations' email listservs. It is worth mentioning that the 1,736 completed surveys may not contain all the variables in this study. It is due to URI IRB regulation that requests that all questions except the screening questions are optional in the survey and the respondents may skip any questions they want.

\subsection{Results}

We use three outcome variables to measure the impact of informational nudges and financial incentives on nudging behavior: WTP for green-certified lawn care contracts,

\footnotetext{
${ }^{3}$ The survey procedure, focus group and all related materials that were shown to the respondents, including the survey itself, were approved by the IRB at the University of Rhode Island.
} 
Likert scale to hire one green-certified lawn care companies and choices to leave information to the three green-certified lawn care companies. Control variables include household lawn care characteristics and respondents' individual characteristics. Table 1.2 lists the descriptive statistics of key variables of this study. We start by reporting the results of the respondents' stated WTP.

\subsubsection{The informational nudges and the stated WTP}

The average WTP $($ Median $=\$ 200$, Mean $=\$ 423.92, \mathrm{~N}=1030)$ stated by the respondents in the informational nudges treatment group is higher than the average $($ Median $=\$ 200$, Mean $=\$ 416.61, \mathrm{~N}=522)$ in the control group. However, the Student $\mathrm{t}-$ Test $(p=0.88)$ does not reject the null hypothesis that the two means are indifferent with each other and Wilcoxon rank-sum test $(p=0.36)$ does not reject its null hypothesis either. Since many factors can affect the WTP, such as lawn acreages or lawn management efforts, which may confound the treatment effect, we specify regression models to control those potential factors and identify confounders if any.

Looking into the data, we find that lawn care spending is a good predictor of the stated WTP. Also, we observe zero WTP values for a significant fraction $(17.43 \%$ in the control group, $15.34 \%$ in the informational nudge treatment group). Thus, we first set up a TOBIT regression model, with the cost in 2016 and experimental design variables as independent variables. An interaction term between the cost variable and the nudge dummy variable is also included to check whether the hypothesized WTP premium nudging by the salient information is affixed to the previous year's lawn care spending. The first column of Table 1.3 presents the results of this TOBIT model. It seems informational nudges can increase the WTP significantly $(p<10 \%)$ when spending on lawn care is not too high on 
the lawn care. However, the statistically significant negative slope for the interaction term indicates that this premium diminishes as the spending increases. The effect of the informational nudges could be negative when the spending is high enough. The NEP Scale and the SRA Scale is added to the TOBIT model to check whether the two individual characteristics well predict the WTP. We do not find that the two variables statistically significantly affect the WTP. The effect of informational nudges on the WTP still holds ( $p$ $<5 \%)$, even in the third model in which we add all other lawn characteristics and individual characteristics as explanatory variables. (Other variables, such as income and the variable indicating whether households hired lawn care companies to manage their lawn have significant effects on the WTP. However, they are not the focuses of the paper, and we do not report the results on Table 1.3.)

Nevertheless, the informational nudges do not affect the WTP statistically significantly after we restrict the observations to a portion of the sample. In the fourth regression model $(\mathrm{N}=675)$, we drop the WTP observations if the respondents state that they are slightly unlikely, moderately unlikely or extremely unlikely to hire green-certified lawn care companies. We can observe that informational nudges still have a positive effect and their interaction with the cost slopes down, but the coefficients of the two variables are not significant anymore (fourth column of Table 1.3). It suggests that some respondents might be nudged to state high WTP even they are not likely to obtain contracts. It also indicates that the hypothetical WTP premium for informational nudges in our survey may have a gap between the actual one.

Taken together, we find that the informational nudges can increase people's hypothetical WTP to for green-certified lawn care contract. However, the effect of the 
nudges might be different from person to person. Evidence even shows that the informational nudges might lower people's WTP when their lawn care bill is supposed to be high. We apply the same analysis to figure out the possible different treatment effects between offering Nudge 1 only and offering Nudge 1 and Nudge 2 together. However, no significant differences are detected.

\subsubsection{The informational nudges, financial incentives and stated Likert scales}

The Likert scales to hire the green-certified lawn care companies are used to measure the effect of informational nudges and financial incentives together. As stated before, we asked the Likert scales question after the respondents randomly received the financial incentives. The Likert scales have six levels, and we assign a value of one to the Likert scales if they state that they are extremely unlikely to hire green-certified lawn care companies and a value of six for extremely likely.

We first run a Pearson's Chi-square test the independence between informational nudges and the Likert scales. The result rejects the hypothesis that the two variables are independent of each other at $10 \%$ level $\left(\chi^{2}=11.02, p=0.051\right)$. Surprisingly, the Chi-Square test for the independence between financial incentives ( $=1$ if any incentives are given) and the Likert scales do not reject the null hypothesis $\left(\chi^{2}=1.99, p=0.85\right)$. We further replace the financial incentives variable with another categorical one, which assigns value zero to the no incentive group, value one to low incentive group and value two to high incentive group, in the test. Its result reject the hypothesis at $10 \%$ level $\left(\chi^{2}=16.79, p=0.08\right)$, however. We conjecture that the two types of financial incentives may have different effects in the stated Likert Scales. Thus, in the following regression analysis, we 
differentiate the financial incentives as the low financial incentive and the high financial incentive.

We use ordered logistic regressions (OLOGIT) to estimate the treatment effect of the informational nudges and the financial incentives since our dependent variables are ordered categorical variables and our data meet all the assumptions of ordered logistic models. We first run the regression with the design variables as independent variables, including the interaction terms of the two treatment variables and the variable defining the recruitment channels. The results of the first model (column 1, Table 1.4a) indicates the stated likelihood to hire a green-certified lawn care companies is statistically significantly higher $(p<0.01)$ in the groups presented with the high financial incentive, the informational nudges, and the interaction of informational nudge and the high financial incentive than in the group with no financial incentives and no nudges. However, presenting the low financial incentive alone does not change the likelihood statistically significant. The coefficient of the interaction term of the low financial incentive and the informational nudges is only significant at $10 \%$ level $(p=0.051)$, which indicates that the low financial incentive may crowd out the likelihood crowded in by the informational nudges. Interestingly, it seems the stated likelihood from the respondents recruited from the Qualtrics panel is higher than that reported by the respondents recruited from the local organizations' email listservs.

We further added the NEP Scale and the SRA Scale into the second model as explanatory variables. Both variables have statistically significant effects $(p<0.01$, column 2, Table 1.4a) on the stated likelihood. The results are robust when we add lawn care characteristics and the other individual characteristics into the third and fourth models 
(column 3 and column 4, Table 1.4a). The effects of high incentive and the informational nudges also persist with more independent variables according to Table 1.4a.

We present marginal effects comparison of six interactions of the informational nudges and the financial incentives in Table $1.4 \mathrm{~b}$ based on the fourth OLOGIT model. The baseline is no informational nudges and no financial incentives. The results show that the informational nudges, the financial incentives, and their interactions statistically significantly decrease the respondents' probabilities of choosing "extremely unlikely" and "moderately unlikely" and increase the chances of answering "slightly likely", "moderately likely" and "extremely likely". For example, the sampled respondents are $10 \%$ less likely to report that they are extremely unlikely hire green-certified companies if the informational nudges are presented (the third cell of column 1 in Table 1.4b). The sampled respondents are about $4 \%$ more likely to state that they are extremely likely to hire greencertified companies if we provide them the informational nudges and the high incentive (the sixth cell of column 6). Conducting more pairwise comparisons between marginal effects of interactions of the informational nudges and the informational nudges, we find systemic differences in the effects of the financial incentives conditional on the presence of the informational nudges. When we do not show the informational nudges to the respondents, three level of incentives (no incentive, low incentive, high incentive) have statistically significantly different marginal effects with each other. The results coincide with the neo-classical economic model predictions in that more incentives induce fewer people to choose Likert Scale 1-2 and more people to choose Likert Scale 4-6. However, when we reveal the informational nudges, the levels of incentives do not have statistically significant margins effects at any Likert Scale (1-6) at 5\% level. 
Based on the results of Tables $1.4 \mathrm{a}$ and $1.4 \mathrm{~b}$, we conclude that the informational nudges and the financial incentives increase the respondents' stated likelihood to hire green-certified lawn care companies and the size of the financial incentives matter. Predicted marginal effects tell us that the financial incentives do not play a significant role in the respondents' stated likelihood to hire green certified lawn care companies when they are exposed the informational nudges. Also, people who are more environmentally friendly and altruistic are more likely to show interests in the green-certified lawn care companies. Nevertheless, will they act? The following part analyzes the determinants of the information acquisition requirements variables.

\subsubsection{The informational nudges, financial incentives and the first step}

Table 1.5 summarizes the rate of the respondents who want the green-certified lawn care companies to contact them and give free estimates. It seems the informational nudges increases the action rate no matter whether there are incentives. However, based on Chisquare tests (Column 3, Table 1.5), the action rate is dependent on the nudge treatment at $10 \%$ level when no financial incentives are provided, not in the cases when we give incentives to the respondents. It suggests that the informational nudges and incentives may substitute each other when nudging the respondents to act to obtain a green-certified lawn care contract.

We further use binomial logistic regression (LOGIT) models to identify the treatment effects, and we show the results in Table 1.6. The odd columns show coefficients of the regressions and the even columns presents the average marginal effects of the variables. The experiment design variables are predictors of the probability of actions in the first model $\left(\chi^{2}=12.93, p=0.04\right)$. The results (Column 1 , Table 1.6) show that both 
informational nudges, financial incentives and their interactions increase the log odds of leaving their contact information for the green-certified lawn care companies. For example, the informational nudges can increase the log odds of actions by 0.875 at $10 \%$ level (column 1, row 3 ). The probability of taking the first step increases by 6 percentage points if the informational nudges are provided (column 2, row 3), which means the nudges have more than doubled the probability of leaving private contact information. Adding the financial incentives with the informational nudges, however, does not increase the probability of leaving private information, which is similar to the findings from the stated Likert scale variable. Surprisingly, we also find that the respondents from Qualtrics Panel are less likely to act even they stated that they are more likely to hire a green-certified lawn care contract (Table 1.4a, row 6).

We add the NEP Scale and the SRA Scale in the second model and find that the respondents' probability to take the first step increases when the NEP Scales or the SRA Scale increases. Financial incentives and their interactions with the informational nudges still have significant effects in this model, but without financial incentives, the informational nudges have a limited effect [coefficient $=0.778, p=0.13$; average marginal effect (compared to the baseline) $=0.052, p=0.09]$. It suggests that the informational nudges can have indirect effects on the action rate through NEP Scales and high SRA Scales. When we add the interaction terms between treatments and NEP/SRA scales in the third models, we find that the average marginal effects increase in the third model by half point percent, and the trend holds when we add more lawn care characteristics and individual characteristics to the regression in the fourth model. 
We further look into the indirect marginal effects of the informational nudges and the financial incentives by comparing the predicted marginal effects at specific NEP Scale and SRA Scale values. We find that the indirect effect of the financial incentives increases substantially when the NEP Scale is high (Figure 1.1). It may suggest that financial incentives can motivate homeowners with pro-environmental attitudes. We find no statistically significant indirect effect of the informational nudges coming through the NEP and SRA Scales. Worth to mention, we cannot differentiate the effect of presenting Nudge 1 and Nudge 2 together on the action from the effect of showing Nudge 1 alone.

The results clearly demonstrate that both information and financial incentives increase the chance of people leaving their contact information for the lawn care companies. However, the results suggest that they are substitutes when implemented together. Evidence also shows that the financial incentives may nudge people with high NEP Scales to act and the size of the financial incentives matters. This result seems to support the argument by Gneezy et al. [2011] that the high financial incentive may have a large price effect so that it can offset the crowd-out effect and increases the action to obtain green-certified lawn care contracts.

\subsection{Conclusion}

This study empirically investigates the impact of the informational nudges on promoting voluntary behavior to adopt BMPs indirectly to reduce NPS pollution in a lawn care market setting. The green certification program by RIDEM integrates BMPs, a list of environmental goods, into the lawn care contracts which are market goods. In collaboration with the local lawn care industry in Rhode Island, we conduct a field experiment and obtain two types of outcomes to measure the residents' demand for the green certification. The 
first type belongs to the stated preferences family. It includes the measure of people's WTP for and the reported likelihood to obtain the green-certified lawn care contracts. The second type is one type of revealed preferences, and it captures whether individuals take the first step towards a green-certified lawn care contracts by leaving their private contact information for the lawn care companies.

The analysis of three outcome variables indicates that the information nudges can induce behavior change conditionally. Specifically, the respondents report WTP premiums and a higher likelihood to sign contracts when we present them the informational nudges. The information nudges also induce more respondents to take the first step to contract. However, when we show the informational nudges and financial incentives together, we detect that they substitute each other especially when the financial incentives are small. Our findings also suggest that the effect of the informational nudges differs across different types of people. The WTP premiums diminish as the spending on the lawn care increase; the informational nudges have a more significant effect when people have low NEP Scales. Conversely, financial incentives have a larger effect when people have high NEP Scales.

Additional results show that the financial incentives can nudge pro-social behavior to some extent, particularly when the incentives are significant. However, when managing NPS pollution from the lawns, an environmental regulator has to face many small private landowners. It makes the financial incentives unfeasible under a limited budget. When incentives substitute the effect of the informational nudges and large financial incentives are not an option, the voluntary programs to reduce NPS pollution should focus on the nudging strategies by more efficient education programs and offering more environmentally friendly choices to the public. 


\section{Acknowledgments}

This material is based upon work supported in part by the RI Agricultural Experiment Station Hatch Regional - RIO015-W3133 and National Science Foundation EPSCoR Track-2 Cooperative Agreement IIA-1330406, Collaborative Research: North East Water Resources Network. 


\section{References}

Ahmed, Rasha, and Kathleen Segerson. 2011. "Collective Voluntary Agreements to Eliminate Polluting Products." Resource and Energy Economics 33 (3): 572-88. doi:10.1016/j.reseneeco.2011.01.002.

Aldrovandi, Silvio, Gordon DA Brown, and Alex M. Wood. 2015. "Social Norms and Rank-Based Nudging: Changing Willingness to Pay for Healthy Food." Journal of Experimental Psychology: Applied 21 (3): 242.

Allcott, Hunt. 2011. "Social Norms and Energy Conservation.” Journal of Public Economics, Special Issue: The Role of Firms in Tax Systems, 95 (9-10): 108295. doi:10.1016/j.jpubeco.2011.03.003.

Alpízar, Francisco, and Peter Martinsson. 2012. "Paying the Price of Sweetening Your Donation: Evidence from a Natural Field Experiment." Economics Letters 114 (2): 182-85. doi:10.1016/j.econlet.2011.10.008.

Alpízar, Francisco, Till Requate, and Albert Schram. 2004. "Collective versus Random Fining: An Experimental Study on Controlling Ambient Pollution."

Environmental and Resource Economics 29 (2): 231-52. doi:10.1023/B:EARE.0000044608.66145.0c.

Attari, Shahzeen Z., David H. Krantz, and Elke U. Weber. 2016. "Energy Conservation Goals: What People Adopt, What They Recommend, and Why." Judgment and Decision Making 11 (4): 342.

Bakacs, Michele E., Mike Haberland, Salvatore S. Mangiafico, Aileen Winquist, Christopher C. Obropta, Amy Boyajian, and Sara Mellor. 2013. "Rain Barrels: A Catalyst for Change?" Journal of Extension 51 (3). https://www.joe.org/joe/2013june/rb6.php.

Ballentine, Jane.2005. "Chesapeake Bay Social Marking Initiative 2004-2005 Final Report", Chesapeake Bay Program https://cfpub.epa.gov/npstbx/relatedpopup.cfm?RelatedMaterialID=118

Bertrand, Marianne, and Adair Morse. 2011. "Information Disclosure, Cognitive Biases, and Payday Borrowing." The Journal of Finance 66 (6): 1865-93. doi:10.1111/j.1540-6261.2011.01698.x.

Brandon, Duane M., James H. Long, Tina M. Loraas, Jennifer Mueller-Phillips, and Brian Vansant. 2013. "Online Instrument Delivery and Participant Recruitment Services: Emerging Opportunities for Behavioral Accounting Research." Behavioral Research in Accounting 26 (1):1-23. https://doi.org/10.2308/bria50651.

Costa, Dora L., and Matthew E. Kahn. 2013. 'Energy Conservation 'Nudges' and Environmentalist Ideology: Evidence from a Randomized Residential Electricity Field Experiment." Journal of the European Economic Association 11 (3): 680702. doi:10.1111/jeea.12011.

Craig, Robin Kundis, and Anna M. Roberts. 2015. "When Will Governments Regulate Nonpoint Source Pollution? A Comparative Perspective." https://papers.ssrn.com/sol3/papers.cfm?abstract_id=2514350.

Depositario, Dinah Pura T., Jr. Nayga Rodolfo M., Ximing Wu, and Tiffany P. Laude. 2009. "Effects of Information on Consumers' Willingness to Pay for Golden Rice.” Asian Economic Journal 23 (4): 457-76. doi:10.1111/j.1467- 
8381.2009.02021.x.

Dietz, Michael E., John C. Clausen, and Karen K. Filchak. 2004. "Education and Changes in Residential Nonpoint Source Pollution." Environmental Management 34 (5): 684-90. doi:10.1007/s00267-003-0238-4.

Dunlap, Riley E., Kent D. Van Liere, Angela G. Mertig, and Robert Emmet Jones. 2000. "New Trends in Measuring Environmental Attitudes: Measuring Endorsement of the New Ecological Paradigm: A Revised NEP Scale." Journal of Social Issues 56 (3): 425-42. doi:10.1111/0022-4537.00176.

Eisenhauer, Brian W., Nicholas Stevenson, Julia Peterson. 2010. "Changing homeowner's lawn care behavior to reduce nutrient losses in New England's urbanizing watersheds Social Science Results Summary." University of New Hampshire Cooperative Extension report.

Eur. Environ. Agency (EEA). 2007. 'Europe's Environment-The Fourth Assessment, 2007. "Copenhagen: EEA

EPA. 2005. "National management measures to control nonpoint source pollution from forestry." EPA-841-B-05-001, EPA, Washington, DC

EPA. 2007. "National Water Quality Inventory: Report to Congress.” EPA 841-R-07001, EPA, Washington, DC

Fellner, Gerlinde, Rupert Sausgruber, and Christian Traxler. 2013. “Testing Enforcement Strategies in the Field: Threat, Moral Appeal and Social Information." Journal of the European Economic Association 11 (3): 634-60. doi:10.1111/jeea.12013.

Ferraro, Paul J., and Agnes Kiss. 2002. "Direct Payments to Conserve Biodiversity." Science 298 (5599): 1718-19. doi:10.1126/science.1078104.

Gneezy, Uri, Stephan Meier, and Pedro Rey-Biel. 2011. "When and Why Incentives (Don't) Work to Modify Behavior." The Journal of Economic Perspectives 25 (4): 191-209.

Griffin, Ronald C., and Daniel W. Bromley. 1982. "Agricultural Runoff as a Nonpoint Externality: A Theoretical Development." American Journal of Agricultural Economics 64 (3): 547-52. doi:10.2307/1240648.

Hansen, Pelle Guldborg. 2015. "The Definition of Nudge and Libertarian Paternalism: Does the Hand Fit the Glove?" European Journal of Risk Regulation, no. 1: 1-20.

Hobbie, Sarah E., Jacques C. Finlay, Benjamin D. Janke, Daniel A. Nidzgorski, Dylan B. Millet, and Lawrence A. Baker. 2017. "Contrasting Nitrogen and Phosphorus Budgets in Urban Watersheds and Implications for Managing Urban Water Pollution." Proceedings of the National Academy of Sciences 114 (16): 4177-82. doi:10.1073/pnas.1618536114.

Jenkins, Virginia. 2015. The Lawn: A History of an American Obsession. Smithsonian Institution.

Kelly, Meghan, Samuel Little, Kaitlin Phelps, Carrie Roble, and Michaela Zint. 2012. "Watershed Outreach Professionals' Behavior Change Practices, Challenges, and Needs." Applied Environmental Education \& Communication 11 (1): 35-52. doi:10.1080/1533015X.2012.728066.

Lacetera, Nicola, Mario Macis, and Robert Slonim. 2012. "Will There Be Blood? Incentives and Displacement Effects in Pro-Social Behavior." American Economic Journal: Economic Policy 4 (1): 186-223. doi:10.1257/pol.4.1.186.

Law, Neely, Lawrence Band, and Morgan Grove. 2004. "Nitrogen Input from Residential 
Lawn Care Practices in Suburban Watersheds in Baltimore County, MD." Journal of Environmental Planning and Management 47 (5): 737-55.

doi:10.1080/0964056042000274452.

Levitt, Steven D., and John A. List. 2007. "What Do Laboratory Experiments Measuring Social Preferences Reveal about the Real World?" The Journal of Economic Perspectives 21 (2): 153-74.

Mathios, Alan D. 2000. "The Impact of Mandatory Disclosure Laws on Product Choices: An Analysis of the Salad Dressing Market." The Journal of Law and Economics 43 (2): 651-78. doi:10.1086/467468.

Milesi, C., C. D. Elvidge, J. B. Dietz, B. T. Tuttle, R. R. Nemani, and S. W. Running. 2005. "A Strategy for Mapping and Modeling the Ecological Effects of US Lawns.” J. Turfgrass Manage 1: 83-97.

Nielson, Lisa, and Courtland L. Smith. 2005. "Influences on Residential Yard Care and Water Quality: Tualatin Watershed, Oregon.” JAWRA Journal of the American Water Resources Association 41 (1): 93-106.

Otto, Philipp E., and Friedel Bolle. 2011. "Multiple Facets of Altruism and Their Influence on Blood Donation." The Journal of Socio-Economics 40 (5): 558-63. doi:10.1016/j.socec.2011.04.010.

Pellerano, José A., Michael K. Price, Steven L. Puller, and Gonzalo E. Sánchez. 2016. "Do Extrinsic Incentives Undermine Social Norms? Evidence from a Field Experiment in Energy Conservation." Environmental and Resource Economics, November, 1-16. doi:10.1007/s10640-016-0094-3.

RIDEM. 2014 “303(d) list: List of Impaired Waters.” http://www.dem.ri.gov/pubs/303d/303d14d.pdf

Rissman, Adena R., and Stephen R. Carpenter. 2015. "Progress on Nonpoint Pollution: Barriers \&amp; Opportunities.” Daedalus 144 (3): 35-47. doi:10.1162/DAED_a_00340.

Rousseau, Sandra, and Liesbet Vranken. 2011. "The Impact of Information on the Willingness-to-Pay for Labeled Organic Food Products." https://papers.ssrn.com/sol3/papers.cfm?abstract_id=1859285.

Rushton, Philippe J., Roland D. Chrisjohn, and G. Cynthia Fekken. 1981. "The Altruistic Personality and the Self-Report Altruism Scale." Personality and Individual Differences 2 (4): 293-302. doi:10.1016/0191-8869(81)90084-2.

Schubert, Christian. 2017. "Green Nudges: Do They Work? Are They Ethical?" Ecological Economics 132 (February): 329-42. doi:10.1016/j.ecolecon.2016.11.009.

Segerson, Kathleen. 1988. "Uncertainty and Incentives for Nonpoint Pollution Control." Journal of Environmental Economics and Management 15 (1): 87-98. doi:10.1016/0095-0696(88)90030-7.

_. 2013. "Voluntary Approaches to Environmental Protection and Resource Management." Annual Review of Resource Economics 5 (1): 161-80. doi:10.1146/annurev-resource-091912-151945.

Shortle, James S., Marc Ribaudo, Richard D. Horan, and David Blandford. 2012. "Reforming Agricultural Nonpoint Pollution Policy in an Increasingly BudgetConstrained Environment." Environmental Science \& Technology 46 (3): 131625. doi:10.1021/es2020499. 
Spence, Porchè L., Deanna L. Osmond, Wesley Childres, Joshua L. Heitman, and Wayne P. Robarge. 2012. "Effects of Lawn Maintenance on Nutrient Losses Via Overland Flow During Natural Rainfall Events1." JAWRA Journal of the American Water Resources Association 48 (5): 909-24. doi:10.1111/j.17521688.2012.00658.x.

Stemle, Adam, Hirotsugu Uchida, and Cathy A. Roheim. 2016. "Have Dockside Prices Improved after MSC Certification? Analysis of Multiple Fisheries." Fisheries Research 182 (October): 116-23. doi:10.1016/j.fishres.2015.07.022.

Sun, Bo, Linxiu Zhang, Linzhang Yang, Fusuo Zhang, David Norse, and Zhaoliang Zhu. 2012. "Agricultural Non-Point Source Pollution in China: Causes and Mitigation Measures.” Ambio 41 (4): 370-79. doi:10.1007/s13280-012-0249-6.

Thaler, Richard H., and Cass R. Sunstein. 2008. "Nudge: Improving Decisions about Health, Wealth, and Happiness." Yale University Press.

Xepapadeas, Anastasios. 2011. "The Economics of Non-Point-Source Pollution.” Annual Review of Resource Economics 3 (1): 355-73. doi:10.1146/annurev-resource083110-115945. 
Table 1.1: Treatment Table

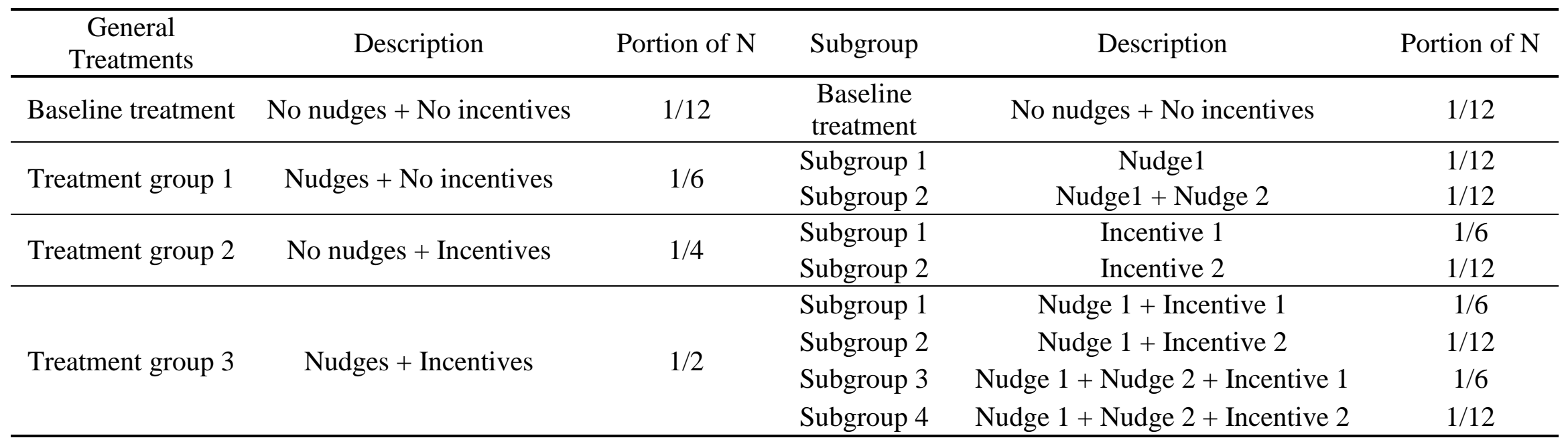


Table 1.2: Descriptive statistics

\begin{tabular}{|c|c|c|c|c|c|}
\hline Variables & Description & $\mathbf{N}$ & Median & Mean & Std. Dev \\
\hline \multicolumn{6}{|c|}{ Panel A. Main outcome variables } \\
\hline WTP & Willingness to pay $(\$)$ for green-certified lawn contracts & 1552 & 200 & 421.5 & 910.75 \\
\hline Likert Scale & Likert scale to hire one ( $1-6 ; 1=$ extremely unlikely; $6=$ extremely likely) & 1591 & 3 & 3.11 & 1.69 \\
\hline Act & Request contact from lawn care companies(dummy, $1=$ request) & 1277 & 0 & 0.12 & 0.32 \\
\hline \multicolumn{6}{|c|}{$\underline{\text { Panel B. Experiment design variables }}$} \\
\hline Nudge 1 & Informational nudge 1 (dummy, = 1 if provided) & 1657 & 1 & 0.67 & 0.47 \\
\hline Nudge 1 only & Informational nudge 1 only (dummy, = 1 if provided) & 1657 & 0 & 0.33 & 0.47 \\
\hline Nudges $1 \& 2$ & Informational nudges 1 and 2 (dummy, = 1 if provided)) & 1657 & 0 & 0.34 & 0.47 \\
\hline Low incentive & Incentive $1,25 \%$ of contract (dummy, = 1 if provided) & 1603 & 1 & 0.5 & 0.5 \\
\hline High incentive & Incentive $2,50 \%$ of contract (dummy, $=1$ if provided) & 1603 & 0 & 0.25 & 0.43 \\
\hline Panel & Panel (dummy, = 1 if respondents comes from Panel) & 1736 & 1 & 0.58 & 0.49 \\
\hline \multicolumn{6}{|c|}{ Panel C. Household lawn care characteristics } \\
\hline Hire & Had a lawn contract in 2016 (dummy, = 1 if had one) & 1736 & 0 & 0.15 & 0.35 \\
\hline Cost in 2016 & Lawn care spending in 2016 & 1641 & 140 & 367.66 & 820.72 \\
\hline Acreage & Lawn acreage & 1648 & 0.5 & 0.7 & 1.22 \\
\hline \# Practices & Number of practices to manage lawns in 2016 & 1716 & 4 & 3.87 & 2.15 \\
\hline \# BMPs & Number of known BMPs applied to lawns in 2016 & 1628 & 3 & 3.47 & 1.58 \\
\hline \multicolumn{6}{|c|}{ Panel D. Respondents' individual characteristics } \\
\hline NEP Scale & New Ecological Paradigm scale (0-6) & 1534 & 3.53 & 3.58 & 0.56 \\
\hline SRA Scale & Self-Report Altruism Scale (0-80) & 1512 & 39 & 39.36 & 12.17 \\
\hline Belief & Number of BMPs that is recognized as effective by the respondents $(0-6)$ & 1616 & 4 & 3.56 & 2.05 \\
\hline Gender & Gender (dummy, $=1$ if female) & 1545 & 1 & 0.65 & 0.48 \\
\hline Age & Age (categorical variable) & 1567 & 4 & 4 & 1.55 \\
\hline Education & Education (categorical variable) & 1569 & 6 & 5.25 & 1.62 \\
\hline Income & Income (categorical variable) & 1492 & 4 & 4.4 & 1.8 \\
\hline
\end{tabular}


Table 1.3: Estimation results of the TOBIT models

\begin{tabular}{|c|c|c|c|c|}
\hline VARIABLES & $\begin{array}{c}(1) \\
\text { WTP }\end{array}$ & $\begin{array}{c}(2) \\
\text { WTP }\end{array}$ & $\begin{array}{c}(3) \\
\text { WTP }\end{array}$ & $\begin{array}{c}(4) \\
\text { WTP }\end{array}$ \\
\hline Cost in 2016 & $\begin{array}{c}0.957 * * * \\
(0.0715)\end{array}$ & $\begin{array}{c}0.955^{* * *} \\
(0.0726)\end{array}$ & $\begin{array}{l}0.845^{* * * *} \\
(0.0867)\end{array}$ & $\begin{array}{l}0.848 * * * \\
(0.0999)\end{array}$ \\
\hline Nudge 1 & $\begin{array}{l}125.7^{*} \\
(67.87)\end{array}$ & $\begin{array}{l}134.9^{*} \\
(69.99)\end{array}$ & $\begin{array}{l}140.5^{* *} \\
(69.87)\end{array}$ & $\begin{array}{c}92.03 \\
(61.27)\end{array}$ \\
\hline Nudge 1 \# Cost in 2016 & $\begin{array}{c}-0.343^{* *} \\
(0.171)\end{array}$ & $\begin{array}{c}-0.346^{* *} \\
(0.174)\end{array}$ & $\begin{array}{c}-0.347 * * \\
(0.161)\end{array}$ & $\begin{array}{l}-0.249 \\
(0.185)\end{array}$ \\
\hline Panel & $\begin{array}{c}78.75 \\
(52.21)\end{array}$ & $\begin{array}{c}64.39 \\
(58.66)\end{array}$ & $\begin{array}{c}63.77 \\
(54.89)\end{array}$ & $\begin{array}{c}17.58 \\
(47.50)\end{array}$ \\
\hline NEP Scale & & $\begin{array}{l}47.70 \\
(39.84)\end{array}$ & $\begin{array}{c}40.58 \\
(41.89)\end{array}$ & $\begin{array}{l}-62.52 \\
(38.53)\end{array}$ \\
\hline SRA Scale & & $\begin{array}{c}0.181 \\
(1.865)\end{array}$ & $\begin{array}{l}-0.426 \\
(1.943)\end{array}$ & $\begin{array}{l}1.706 \\
(1.483)\end{array}$ \\
\hline $\begin{array}{l}\text { Other lawn care } \\
\text { characteristics }\end{array}$ & & & $\checkmark$ & $\checkmark$ \\
\hline $\begin{array}{l}\text { Other individual } \\
\text { characteristics }\end{array}$ & & & $\checkmark$ & $\checkmark$ \\
\hline $\mathrm{N}$ & 1,544 & 1,462 & 1,348 & 675 \\
\hline
\end{tabular}


Table 1.4a: Estimation results of the OLOGIT models

\begin{tabular}{|c|c|c|c|c|}
\hline VARIABLES & $\begin{array}{c}(1) \\
\text { Likelihood } \\
\end{array}$ & $\begin{array}{c}(2) \\
\text { Likelihood }\end{array}$ & $\begin{array}{c}(3) \\
\text { Likelihood } \\
\end{array}$ & $\begin{array}{c}(4) \\
\text { Likelihood }\end{array}$ \\
\hline No nudges \# Low incentive & $\begin{array}{c}0.296 \\
(0.194)\end{array}$ & $\begin{array}{c}0.335^{*} \\
(0.199)\end{array}$ & $\begin{array}{c}0.256 \\
(0.197)\end{array}$ & $\begin{array}{c}0.359^{*} \\
(0.205)\end{array}$ \\
\hline No nudges \# High incentive & $\begin{array}{c}0.786 * * * \\
(0.226)\end{array}$ & $\begin{array}{c}0.778 * * * \\
(0.234)\end{array}$ & $\begin{array}{c}0.758 * * * \\
(0.228)\end{array}$ & $\begin{array}{c}0.827 * * * \\
(0.244)\end{array}$ \\
\hline Nudge 1 \# No incentive & $\begin{array}{c}0.525 * * * \\
(0.196)\end{array}$ & $\begin{array}{c}0.462 * * \\
(0.205)\end{array}$ & $\begin{array}{c}0.377 * \\
(0.201)\end{array}$ & $\begin{array}{c}0.397 * \\
(0.210)\end{array}$ \\
\hline Nudge 1 \# Low incentive & $\begin{array}{l}0.324^{*} \\
(0.182)\end{array}$ & $\begin{array}{c}0.345^{*} \\
(0.189)\end{array}$ & $\begin{array}{c}0.297 \\
(0.186)\end{array}$ & $\begin{array}{c}0.381 * * \\
(0.194)\end{array}$ \\
\hline Nudge 1 \# High incentive & $\begin{array}{c}0.677 * * * \\
(0.200)\end{array}$ & $\begin{array}{c}0.652 * * * \\
(0.205)\end{array}$ & $\begin{array}{c}0.645 * * * \\
(0.202)\end{array}$ & $\begin{array}{c}0.627 * * * \\
(0.214)\end{array}$ \\
\hline Panel & $\begin{array}{c}0.464 * * * \\
(0.0986)\end{array}$ & $\begin{array}{c}0.445 * * * \\
(0.110)\end{array}$ & $\begin{array}{c}0.403 * * * \\
(0.110)\end{array}$ & $\begin{array}{c}0.452 * * * \\
(0.127)\end{array}$ \\
\hline NEP Scale & & $\begin{array}{c}0.773 * * * \\
(0.0987)\end{array}$ & $\begin{array}{c}0.822 * * * \\
(0.101)\end{array}$ & $\begin{array}{c}0.713 * * * \\
(0.103)\end{array}$ \\
\hline SRA Scale & & $\begin{array}{c}0.0167 * * * \\
(0.00445)\end{array}$ & $\begin{array}{l}0.0139 * * * \\
(0.00454)\end{array}$ & $\begin{array}{c}0.0176^{* * * *} \\
(0.00469)\end{array}$ \\
\hline Lawn care characteristics & & & $\checkmark$ & $\checkmark$ \\
\hline $\begin{array}{l}\text { Other individual } \\
\text { characteristics }\end{array}$ & & & & $\checkmark$ \\
\hline Observations & 1,590 & 1,501 & 1,436 & 1,368 \\
\hline
\end{tabular}


Table 1.4b: Average marginal effect comparison

\begin{tabular}{|c|c|c|c|c|c|c|}
\hline VARIABLES & $\begin{array}{c}\text { Extremely } \\
\text { unlikely } \\
1\end{array}$ & $\begin{array}{c}\text { Moderately } \\
\text { unlikely } \\
2\end{array}$ & $\begin{array}{c}\text { Slightly } \\
\text { unlikely } \\
3\end{array}$ & $\begin{array}{c}\text { Slightly } \\
\text { likely } \\
4\end{array}$ & $\begin{array}{c}\text { Moderately } \\
\text { likely } \\
5\end{array}$ & $\begin{array}{c}\text { Extremely } \\
\text { likely } \\
6\end{array}$ \\
\hline No nudges \# Low incentive ${ }^{\psi}$ & $\begin{array}{l}-0.0792 * \\
(0.0409)\end{array}$ & $\begin{array}{c}-0.00811 * * \\
(0.00380)\end{array}$ & $\begin{array}{c}0.00206 \\
(0.00221)\end{array}$ & $\begin{array}{l}0.0272 * \\
(0.0146)\end{array}$ & $\begin{array}{c}0.0377 * * \\
(0.0186)\end{array}$ & $\begin{array}{l}0.0204 * * \\
(0.00988)\end{array}$ \\
\hline No nudges \# High incentive ${ }^{\psi}$ & $\begin{array}{c}-0.173 * * * \\
(0.0441)\end{array}$ & $\begin{array}{c}-0.0289 * * * \\
(0.00848)\end{array}$ & $\begin{array}{l}-0.00505 \\
(0.00458)\end{array}$ & $\begin{array}{c}0.0494 * * * \\
(0.0138)\end{array}$ & $\begin{array}{c}0.0961 * * * \\
(0.0245)\end{array}$ & $\begin{array}{c}0.0617 * * * \\
(0.0178)\end{array}$ \\
\hline Nudge 1 \# No incentive ${ }^{\psi}$ & $\begin{array}{l}-0.101 * * \\
(0.0411)\end{array}$ & $\begin{array}{c}-0.0117 * * * \\
(0.00429)\end{array}$ & $\begin{array}{c}0.00152 \\
(0.00232)\end{array}$ & $\begin{array}{c}0.0336^{* *} \\
(0.0144)\end{array}$ & $\begin{array}{c}0.0495 * * * \\
(0.0191)\end{array}$ & $\begin{array}{c}0.0276^{* *} \\
(0.0107)\end{array}$ \\
\hline Nudge 1 \# Low incentive ${ }^{\psi}$ & $\begin{array}{c}-0.0977 * * \\
(0.0388)\end{array}$ & $\begin{array}{c}-0.0112 * * * \\
(0.00354)\end{array}$ & $\begin{array}{c}0.00163 \\
(0.00226)\end{array}$ & $\begin{array}{c}0.0328 * * \\
(0.0139)\end{array}$ & $\begin{array}{c}0.0479 * * * \\
(0.0176)\end{array}$ & $\begin{array}{c}0.0266 * * * \\
(0.00939)\end{array}$ \\
\hline Nudge 1 \# High incentive ${ }^{\psi}$ & $\begin{array}{c}-0.138 * * * \\
(0.0409)\end{array}$ & $\begin{array}{c}-0.0196 * * * \\
(0.00542)\end{array}$ & $\begin{array}{l}-0.000855 \\
(0.00285)\end{array}$ & $\begin{array}{c}0.0432 * * * \\
(0.0140)\end{array}$ & $\begin{array}{c}0.0723 * * * \\
(0.0201)\end{array}$ & $\begin{array}{c}0.0430 * * * \\
(0.0122)\end{array}$ \\
\hline
\end{tabular}


Table 1.5: Action rate by treatments

\begin{tabular}{cccc}
\hline & No Nudges & Nudges & Nudges vs. Act $(p$-value $)$ \\
\hline No Incentives & $4.76 \%$ & $10.67 \%$ & $\chi^{2}=3.19, p=0.07$ \\
Low Incentives & $11.11 \%$ & $12.13 \%$ & $\chi^{2}=0.14, p=0.70$ \\
High Incentives & $14.00 \%$ & $16.67 \%$ & $\chi^{2}=0.36, p=0.54$ \\
\hline
\end{tabular}


Table 1.6: Estimation results of the LOGIT models

\begin{tabular}{|c|c|c|c|c|c|c|c|c|}
\hline \multirow{2}{*}{$\begin{array}{l}\text { Variables } \\
\text { No nudges \# Low incentive }{ }^{\psi}\end{array}$} & \multicolumn{2}{|c|}{$\begin{array}{l}\text { (1) } \\
\text { Act }\end{array}$} & \multicolumn{2}{|c|}{$\begin{array}{l}\text { (2) } \\
\text { Act }\end{array}$} & \multicolumn{2}{|c|}{$\begin{array}{l}\text { (3) } \\
\text { Act }\end{array}$} & \multicolumn{2}{|c|}{$\begin{array}{l}\text { (4) } \\
\text { Act }\end{array}$} \\
\hline & $\begin{array}{c}\text { Coef. } \\
0.924^{*} \\
(0.509)\end{array}$ & $\begin{array}{c}\text { A.M.E. } \\
0.0642 * * \\
(0.0297)\end{array}$ & $\begin{array}{c}\text { Coef. } \\
0.904 * \\
(0.518)\end{array}$ & $\begin{array}{c}\text { A.M.E. } \\
0.0640^{* *} \\
(0.0313)\end{array}$ & $\begin{array}{c}\text { Coef. } \\
1.361 \\
(2.722)\end{array}$ & $\begin{array}{c}\text { A.M.E. } \\
0.0732 * * \\
(0.0310)\end{array}$ & $\begin{array}{c}\text { Coef. } \\
0.329 \\
(2.823)\end{array}$ & $\begin{array}{c}\text { A.M.E. } \\
0.0876^{* * *} \\
(0.0324)\end{array}$ \\
\hline No nudges \# High incentive ${ }^{\psi}$ & $\begin{array}{c}1.181 * * \\
(0.544)\end{array}$ & $\begin{array}{c}0.0922 * * \\
(0.0403)\end{array}$ & $\begin{array}{l}1.120 * * \\
(0.557)\end{array}$ & $\begin{array}{c}0.0870 * * \\
(0.0411)\end{array}$ & $\begin{array}{l}-0.101 \\
(3.020)\end{array}$ & $\begin{array}{l}0.107 * * \\
(0.0415)\end{array}$ & $\begin{array}{l}-0.388 \\
(3.261)\end{array}$ & $\begin{array}{c}0.118 * * * \\
(0.0437)\end{array}$ \\
\hline Nudge 1 \# No incentive ${ }^{\psi}$ & $\begin{array}{l}0.875^{*} \\
(0.510)\end{array}$ & $\begin{array}{c}0.0594 * * \\
(0.0293)\end{array}$ & $\begin{array}{c}0.778 \\
(0.522)\end{array}$ & $\begin{array}{l}0.0521 * \\
(0.0304)\end{array}$ & $\begin{array}{c}3.714 \\
(2.965)\end{array}$ & $\begin{array}{c}0.0578 * * \\
(0.0295)\end{array}$ & $\begin{array}{c}3.192 \\
(2.892)\end{array}$ & $\begin{array}{c}0.0687 * * \\
(0.0304)\end{array}$ \\
\hline Nudge 1 \# Low incentive ${ }^{\psi}$ & $\begin{array}{c}1.009 * * \\
(0.486)\end{array}$ & $\begin{array}{c}0.0728 * * * \\
(0.0264)\end{array}$ & $\begin{array}{c}0.984 * * \\
(0.494)\end{array}$ & $\begin{array}{c}0.0722 * * * \\
(0.0278)\end{array}$ & $\begin{array}{c}2.711 \\
(2.472)\end{array}$ & $\begin{array}{c}0.0776 * * * \\
(0.0266)\end{array}$ & $\begin{array}{c}2.677 \\
(2.557)\end{array}$ & $\begin{array}{c}0.0865 * * * \\
(0.0277)\end{array}$ \\
\hline Nudge 1 \# High incentive ${ }^{\psi}$ & $\begin{array}{c}1.385^{* * * *} \\
(0.497)\end{array}$ & $\begin{array}{c}0.118 * * * \\
(0.0328)\end{array}$ & $\begin{array}{c}1.396^{* * * *} \\
(0.505)\end{array}$ & $\begin{array}{c}0.122 * * * \\
(0.0341)\end{array}$ & $\begin{array}{c}1.466 \\
(2.734)\end{array}$ & $\begin{array}{c}0.125 * * * \\
(0.0330)\end{array}$ & $\begin{array}{c}0.744 \\
(2.866)\end{array}$ & $\begin{array}{c}0.111 * * * \\
(0.0331)\end{array}$ \\
\hline Panel & $\begin{array}{c}-0.350 * * \\
(0.174)\end{array}$ & $\begin{array}{c}-0.0365^{* *} * \\
(0.0181)\end{array}$ & $\begin{array}{c}-0.541 * * * \\
(0.192)\end{array}$ & $\begin{array}{c}-0.0556^{* * * *} \\
(0.0198)\end{array}$ & $\begin{array}{c}-0.576 * * * \\
(0.193)\end{array}$ & $\begin{array}{c}-0.0584 * * * * \\
(0.0197)\end{array}$ & $\begin{array}{c}-0.771 * * * \\
(0.243)\end{array}$ & $\begin{array}{c}-0.0742 * * * \\
(0.0234)\end{array}$ \\
\hline NEP Scale & & & $\begin{array}{c}0.692 * * * \\
(0.163)\end{array}$ & $\begin{array}{c}0.0710 * * * \\
(0.0167)\end{array}$ & $\begin{array}{c}1.239 * * \\
(0.484)\end{array}$ & $\begin{array}{c}0.0775 * * * \\
(0.0173)\end{array}$ & $\begin{array}{c}0.890 * * \\
(0.442)\end{array}$ & $\begin{array}{c}0.0558 * * * \\
(0.0191)\end{array}$ \\
\hline SRA Scale & & & $\begin{array}{l}0.0165 * * \\
(0.00726)\end{array}$ & $\begin{array}{l}0.00169 * * \\
(0.000747)\end{array}$ & $\begin{array}{c}-0.00335 \\
(0.0192)\end{array}$ & $\begin{array}{l}0.00187 * * \\
(0.000766)\end{array}$ & $\begin{array}{c}-0.00843 \\
(0.0233)\end{array}$ & $\begin{array}{c}0.00107 \\
(0.000854)\end{array}$ \\
\hline Treatments \# NEP scale & & & & & $\checkmark$ & & $\checkmark$ & \\
\hline Treatments \# SRA scale & & & & & $\checkmark$ & & $\checkmark$ & \\
\hline Lawn care characteristics & & & & & & & $\checkmark$ & \\
\hline Other individual characteristics & & & & & & & $\checkmark$ & \\
\hline Observations & 1,276 & & 1,200 & & 1,200 & & 1,079 & \\
\hline
\end{tabular}

Robust standard errors in parentheses for the coefficient column and delta method standard errors in parentheses for marginal effect column. $* * * p<0.01, * * p<0.05, * p<0.1$. $\Psi$ : Baseline category is the treatment without nudges or incentives. 


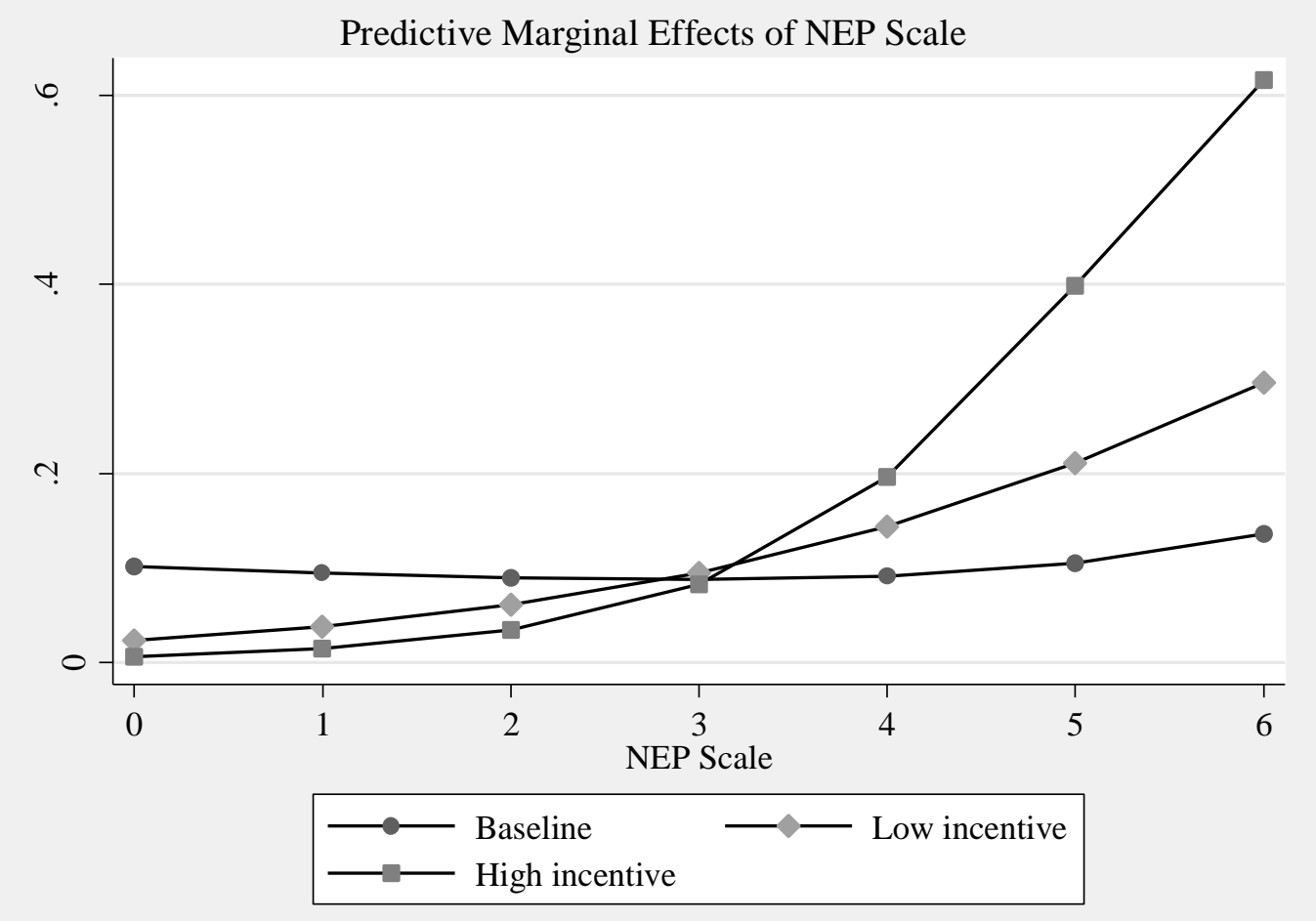

Figure 1.1: Indirect effects of the financial incentives through NEP Scale 


\title{
MANUSCRIPT-2
}

\section{Uncertainty and nonpoint source polluters' behavior under an ambient tax policy}

(To be submitted)

\author{
by \\ Haoran Miao a, Todd Guilfoos ${ }^{\text {a }}$, and Emi Uchida ${ }^{\text {a }}$ \\ ${ }^{a}$ Department of Environmental and Natural Resource Economics, University of Rhode Island, Kinston, RI
}

\begin{abstract}
Ambient-based policy instruments are one approach to regulate nonpoint source pollution. A series of experimental studies have investigated the advantages and disadvantages of the policy under strategic uncertainty but not environmental uncertainty. This study implements a laboratory experiment to further explore the ambient tax policy under strategic uncertainty combined with environmental uncertainty at the individual level. The ability to observe other polluters' actions also varies in the experiment. The results indicate that environmental uncertainty at the individual level does not affect the overall efficiency of the ambient tax policy, but induces under-abatement. Evidence suggests that the nonpoint source polluters tend to be risk-seeking under individual-level environmental uncertainty when given group incentives. Additionally, we find that the ability to observe other group members' behavior may cause cooperative behavior even in a non-cooperative experiment setting.
\end{abstract}

JEL Classification: C91, Q52, Q53, D01

Keywords: Nonpoint Source Pollution, Environmental Uncertainty, Information Disclosure, and Laboratory Experiment 


\subsection{Introduction}

Ambient-based policy instruments proposed by economists [e.g., Segerson, 1988; Xepapadeas, 1992] have shown promise to reduce nonpoint source (NPS) pollution and to increase social efficiency through a series of laboratory economic experiments [e.g., Spraggon, 2002; Cochard, Willinger, and Xepapadeas, 2005; Suter et. al, 2008]. Ambientbased policy instruments do not require the regulator to measure individual polluter's emission level. Therefore, those instruments can theoretically bypass the information asymmetry problems between the environmental regulator and individual polluters and solve the group moral hazard and/or adverse selection problems when accurate observations of individual emissions are not feasible [Xepapadeas, 2011].

Previous studies have tested their effectiveness and efficiency and NPS polluters' potential behavior in laboratory experiments in various contexts. For example, Spraggon [2004] examines the ability of lump-sum or proportional fines and bonuses to reduce NPS pollution with heterogeneous agents. The study discovers that proportional fines and bonus (ambient tax/subsidy) are sufficient to achieve group pollution targets, but it also observes inefficiency and inequality when the agents have different capabilities to pollute. Poe et al. [2004] examine behavioral responses to a few ambient-based policy instruments by allowing polluters to cooperate. They find that the participants' behavior deviates substantially from theoretical predictions of non-cooperative settings. They also find that the instruments which give a proportional bonus to the groups lower their emission levels under a pollution target and thus induce over-abatement behavior. The over-compliance behavior also occurs in other experiments] in which subjects are allowed to communicate with each other under a set of ambient-based policy instruments. Suter, Vossler, and Poe 
[2009] utilize realistic dairy farm data to model firms with different sizes in their experiments. They find evidence that shows the heterogeneity of firm sizes generate both desirable treatment effects and unattractive outcomes under the ambient-based tax instrument.

These experimental studies of ambient-based policy, however, examine its behavioral effects only when the participants face strategic uncertainty, that is when the participants are uncertain about other polluters' actions. Several experimental studies introduce stochastic components at the group level, which does not affect individual NPS polluter's emission as environmental uncertainty [e.g., Suter et al., 2008, Suter and Vossler, 2013]. As a matter of fact, the effect of environmental uncertainty under the ambient-based policy, especially its impact on individual behavior, have not been well studied since Spraggon [2002] reports in his experiment that "the Tax-Subsidy and Tax instruments are better able to enforce the standard than the Subsidy and Group Fine instruments with inexperienced subjects under certainty" and "the result is robust to uncertainty". This study explores how ambient tax instruments affect NPS polluters' behavior when environmental uncertainty exists at the individual level using a large-scale laboratory experiment.

Uncertainty is an essential feature of NPS pollution problem [Young et al., 1989], and it is one of the reasons that NPS pollution is difficult to regulate. In a seminal study, Segerson [1988] developed a theoretical model that shows that ambient-based policy instruments could be used to control NPS pollution in the presence of uncertainty and obstacles of monitoring. We can consider uncertainty and monitoring difficulties both as information problems in NPS pollution control. However, almost every economic 
experiment testing ambient-based policy implements monitoring difficulties and strategic uncertainty but neglects environmental uncertainty, especially at the individual level.

Several studies present environmental uncertainty at the group level in the context of NPS pollution problems [Suter and Vossler, 2013 and Suter et al., 2008]. Other studies [e.g., Cason and Gangadharan, 2013] recognize that environmental uncertainty is an important aspect of NPS pollution problems. However, they do not test the effects of environmental uncertainty because of the existence of the experiment results of Spraggon [2002]. In general, almost all experimental studies examining the ambient-based policy assumes that there are determinate relationships between individual pollution emission levels and individual polluter's actions. This study relaxes this assumption and embeds environmental uncertainty at the individual level to test the effect of environmental uncertainty under the ambient-based policy.

The first motivation of embedding environmental uncertainty at the individual level in our experiment is that the action of one NPS polluter is not the only determinate factor of individual pollution emission levels. Other factors affect the emission levels include temperature, rainfall, and management parameters [Yong et al., 1989]. The implementation of best management practices (BMPs), such as cover crops, contour farming, and conservation tillage, is a standard approach to reducing agricultural NPS pollution. But the effectiveness of these BMPs are not absolute, and extreme weather events can amplify the uncertainty in their effectiveness [Woznicki and Nejadhashemi, 2014].

The second motivation to test the treatment effects of environmental uncertainty at the individual level is to fill the gap of describing decision-making under the ambient-based policy. Existing economic theories such as expected utility theory [Von Neumann \& 
Morgenstern, 1944] and prospect theory [Kahneman and Tversky, 1979] describe how uncertainty or risk affect decision-making in general. Additionally, Faucheux and Froger [1995] studies decision-making under environmental uncertainty. A series of experiments testing these theories suggest that decision-making is related to the framing of the experiments [ e.g., Cohen, Jaffray, and Said, 1987; Elliott, 1998]. In the literature using experiments to examine the ambient-based policy, two studies test the effects of environmental uncertainty at the group level [Spraggon, 2002; Miao et al., 2016]. Spraggon [2002] compares four types ambient-based instruments and find that environmental uncertainty at the group levels does not have significant effects on the efficiency of a subset of ambient-based policies. Miao et al. [2016] test the ambient tax/subsidy instrument in a spatially heterogeneous setting and use uncertain sensor information to model environmental uncertainty at the group levels. They find that the more frequent monitoring leads to efficiency gains. However, these two studies model environmental uncertainty at the group level and their analysis of the effects of environmental uncertainty also focuses at the group level. This study contributes to the current knowledge of effectiveness and efficiency of ambient-based policy by adding analysis of the impact of environmental uncertainty at the individual level on individual behavior.

Another contribution of this study is to test whether NPS polluters' knowledge of other polluters' actions can affect their behavior. Previous studies assume individual NPS polluter's actions are hidden not only from the environmental regulator but also from the group members. Experiment protocols only provide ambient pollution information and related payoffs to the subject. In Spraggon [2013], the number of other polluters and their payoff are also unknown to the individuals in some treatments. We investigate a different 
scenario in which NPS polluters' actions are common knowledge among group members but still hidden from the environmental regulator. This treatment is important because individual polluters in the field may be able to observe the practices implemented by their neighbors. Additionally, studies in the game theory literature suggest that knowing each other's actions may affect players' ability to learn in noise payoff settings. For example, Bereby-Meyer and Roth [2006] find that the subjects' ability to learn to cooperate in a repeated prisoner's dilemma game was substantially diminished when the payoffs were noisy, although players could monitor other players' lagged actions. This study further utilizes this treatment to explores whether knowing others' pollution levels may affect polluting behavior.

We design a new laboratory experiment to test the effectiveness and efficiency of ambient-based policy by understanding the connections between individual-level environmental uncertainty (information disclosure) and NPS polluters' behavior. In the experiment, we disclose the information about other group members' actions to the participants as if they can observe other group members' actions. We follow the design in Suter et al. [2008] but decompose the group uncertainty into individual uncertainty. We also vary the individual uncertainty into three levels: certainty, the low uncertainty, and high uncertainty.The objective of the treatments is to gauge the extent to which uncertainty levels or information disclosure influences social efficiency outcomes and NPS polluters' behavior. The information disclosure treatment block includes two levels: with and without information disclosure. When the information is not disclosed, the subjects only have information on ambient pollution and related payoffs. When the information is disclosed, subjects are given information on other polluters' pollution decisions in the same group. 
We find that the environmental uncertainty at the individual level does not affect the social efficiency. However, environmental uncertainty increased pollution emissions and variation of polluters' behavior. Also, we find that information disclosure reduces social efficiency by increasing collusive behavior.

\subsection{Experimental design}

\subsubsection{Basic design}

This experiment designs NPS pollution problem in a way similar to the theoretical NPS pollution model by Segerson [1988]. We assume I identical farms locate at one small watershed and their activities affect water quality in a common pool in the watershed. Each farm, denoted by $i$, can produce output $y_{i}\left(y_{i} \geq 0\right)$ in each period and Farm $i$ can sell its output at a price $p$. The price is assumed to be fixed across all periods. Then $p^{*} y_{i}$ is the total revenue of Farm $i$. The operation cost of Farm $i$ is represented by $C_{i}=C\left(y_{i}, a_{i}\right)$, where $a_{i}\left(a_{i} \geq 0\right)$ is abatement effort, $C_{y i}=\partial C_{i} / \partial y i>0$ and $C_{a i}=\partial C_{i} / \partial a_{i}>0$.

We use $r_{i}=r\left(y_{i}, a_{i}, \boldsymbol{\varepsilon}\right)$ to denote the emission function of Farm $i$ where $r_{y i}=\partial r_{i} / \partial$ $y i>0$ and $r_{a i}=\partial r_{i} / \partial a_{i}<0$ and $\varepsilon$ is a vector of stochastic variables such as rainfall. The function $x=x\left(r_{1}, r_{2}, \ldots, r_{I}\right) \equiv x(\boldsymbol{a}, \boldsymbol{y}, \boldsymbol{\varepsilon})$ stands for the ambient pollution level of the common pool in the watershed, where $\boldsymbol{a}$ is a vector of abetment efforts of $I$ farms and $\boldsymbol{y}$ is a vector of production of $I$ farms. We assume the total environmental damages of the common pool are a function of the ambient pollution level and equal $D(x)$, where $D(0)=0, D^{\prime}(x)>0$ and $D^{\prime \prime}(x) \geq 0$. 
If the manager of Farm $i$ wants to maximize his/her profit, he/she makes production and abatement decisions based on the following maximization problem if there are no exogenous interventions:

$$
\max _{y i, a i} \text { Profit }=p * y_{i}-C\left(y_{i}, a_{i}\right)
$$

However, from the social planner's point of view, the maximization problem develops to the following expression:

$$
{ }_{y i, a i}^{\max } \text { Profit }=-\mathrm{E}[D(x)]+\sum_{i=1}^{I}\left[p * y_{i}-C\left(y_{i}, a_{i}\right)\right]
$$

Apparently, the optimization problems do not have the same solutions. For optimization problem (1), the manager chooses a zero abatement effort and a production level that makes $C_{y i}=p$, which equalizes the marginal benefits and marginal costs. In contrast, the solutions of the social planner's problems are determined by the following two first-order conditions:

$$
\left\{\begin{array}{c}
-\mathrm{E}\left[D^{\prime}(x) * x_{y_{i}}\right]+p-C_{y_{i}}=0 \\
-\mathrm{E}\left[D^{\prime}(x) * x_{a_{i}}\right]-C_{a_{i}}=0
\end{array}\right.
$$

To make the solutions to the problem (1) and the problem (2) identical, we impose the following tax [Segerson, 1988; Hansen 1998] is on each farm in the small watershed:

$$
T(x)=\left\{\begin{array}{cc}
t_{i} *(x-\bar{x}) & \text { if } x>\bar{x} \\
0 & \text { if } x \leq \bar{x}
\end{array}\right.
$$

where $t_{i}$ is equal to

$$
t_{i}=\frac{\mathrm{E}\left[D^{\prime}(x) * x_{y_{i}}\right]-\mathrm{E}\left[D^{\prime}(x) * x_{a_{i}}\right]}{\mathrm{E}\left[x_{y_{i}}\right]-\mathrm{E}\left[x_{a_{i}}\right]}
$$

If Farm i's manager wants to maximize his/her profit when the environmental regulator imposes the above tax, he/she makes production and abatement decisions based on the following profit maximization problem: 


$$
\max _{y i, a i}=p * y_{i}-C\left(y_{i}, a_{i}\right)-T(x)
$$

Theoretically, Farm $i$ 's decisions about the production and abatement effort will be consistent with the social planner's optimal solution and meet the requirement to maximize the social benefit. Under such policy intervention, the environmental regulator's mission is to measure accurate ambient pollution level when imposing the tax. Therefore, the ambient tax instruments theoretically solve the information problems between the environmental regulator and NPS polluters.

\subsubsection{Parameterization and Treatment description}

In this experiment, the participants take the role of managers of farms, and six of them $(I=6)$ form a group of potential NPS polluters in a small watershed. They have identical profit function, but their emission functions may be different from treatments which will be explained later in this section.

We utilize a $3 \times 2$ experimental design which presents six treatments in total (Table 1). The first treatment dimension is the environmental uncertainty at the individual level. We implement three uncertainty levels, which includes certainty, low uncertainty, and high uncertainty. The second treatment dimension is the information disclosure, which contains no-information disclosure and information disclosure.

The first treatment dimension is different from the previous literature in that the environmental uncertainty is realized at the individual level. We use a certainty treatment block (Treatment A and D), which implies participants can choose their emission levels perfectly, as the baseline of the first treatment dimension. This treatment block is the same as the treatments in most previous literature in that no environmental uncertainty exists. The second treatment block (Treatment B and E) of this dimension is low uncertainty, in 
which participants have individual emission levels with low variation. The third treatment block (Treatment $\mathrm{C}$ and $\mathrm{F}$ ) of this dimension is high uncertainty, in which participants have individual emission levels with high variation.

The second treatment dimension varies from the previous literature in that we include the ability of participants to observe all other group members' actions. The first treatment block in this dimension, including Treatment $\mathrm{A}, \mathrm{B}$, and $\mathrm{C}$, is identical to most previous literature: participants' actions are hidden from not only the environmental regulator but also their group members. The second treatment block, including Treatment $\mathrm{D}, \mathrm{E}$, and $\mathrm{F}$, discloses the action information of group members to the participants.

The profit, emission, damage and ambient functions and their related parameters for all treatments are shown in Table 2.2. From the table, we can see that the profit function is identical across all the treatments and the cost function inside the profit function take the quadratic form of the production levels and the abetment effects, which agrees with assumptions of the cost function in the theoretical design.

The emission functions vary based on uncertainty level. In general, it is determined by participants' chosen production levels and abetment efforts and one stochastic environmental variable. The emission functions have a quadratic form of the production levels and a square root of abatement efforts, which ensure the emission functions are convex in production levels and abatement efforts. We simplify the stochastic environmental vector $\varepsilon$ into one dimension. The stochastic terms are completely independent of production levels and abetment efforts and have means of one. In the certainty treatment, the stochastic term is a constant which equals one. In the low uncertainty treatments, the stochastic term is a normalized (mean $=1$ ) lognormal 
distribution with a small variation parameter. In the high uncertainty treatments, the stochastic term has the same mean as in the low uncertainty treatments, but with a relatively high variation parameter.

The ambient pollution function, damage function, and ambient tax function are mostly drawn from previous literature. The ambient pollution function is the summation of individual emissions. The damage function is a linear function of ambient pollution level. Based on previous theoretical work, a linear function of ambient pollution level in which marginal tax equals marginal damage can meet the requirement of the optimal tax.

We design parameters of all the functions such that all the functions meet all the assumptions in the theoretical design (Table 2.2). We let $\bar{x}$, the threshold of ambient tax, to be zero in our experiment which implies we impose tax on every unit of pollution. Because Suter et al. [2008] suggest that the tax threshold does not affect the abilities of groups to achieve the targeted emissions level and theoretical literature also demonstrate that the threshold of ambient tax is an exogenous variable determined by the regulator [Segerson, 1988], we use zero as the threshold to reduce participants' confusion towards the ambient tax.

The modeling of the stochastic variables is motivated by the fact that nutrient loss from farm field is positively correlated with runoff amount. For example, in a series of high-frequency sensor data drawn from the research results of North East Water Resource Network [Vaughan et al. 2017], the Nitrate export in a watershed in Burlington, Vermont in a two-year period is highly correctly with stormwater yield according to an Ordinal Least Square regression $(\mathrm{R} 2=0.7697)$. Pollutants on the farm filed could have no significant impact on water quality if there is no sufficient runoff. We assume that the volume of runoff 
is a proportion of the rainfall depth. Based on this assumption, we further presume that individual emissions are proportional to rainfall depth. We use monthly summer rainfall data in the past forty years in Burlington area [National Weather Service Forecast Office, National Oceanic and Atmospheric Administration (NOAA)] to fit a distribution and lognormal distribution $[\varepsilon \sim \log N(1.25,0.5)]$ is a good estimation of the rainfall data. To test whether the participants respond to increased variation of rainfall due to other stochastic variables such as climate change, we double the variation parameter of the fitted lognormal distribution and keep the same mean to form a new lognormal distribution to mimic rainfall events with high variation.

The basic design of NPS pollution problem in this experiment presumes the farms choose between two variables, the production level, and abetment efforts. We adopt the idea from Suter et al. [2008] to simplify the participants' choices by identifying a discrete set of production level and abatement effort combinations which together imply a management option. One management option is a set of production level and abatement effort maximizing the pre-tax profit subject to a specific (expected) emission levels (Table 3). Based on such transition, the ambient tax instrument looks more transparent to the participants.

\subsubsection{Experiment protocol}

We ran the experiment at the Policy Simulation Lab of University of Rhode Island (URI) during the fall semester of 2016. A total of 252 students participated in 21 experimental sessions. Most of them were URI undergraduate students, and a small portion of them are graduate students. The majority of the participants did not have previous experience participating in economic experiments. We randomly assign all the participants 
to 42 groups during the experimental sessions. Each group had six participants. We used z-Tree [Fischbacher, 2007] to implement the experiment and record all decisions made by the participants.

The participants gathered in a meeting room before the experiments began. They picked a piece of paper with a seat number to find out where they would be seated. Then they had three minutes to read the consent form for the experiment. After everyone finished reading the consent form, the lead author gave all participants a brief description of the experiment and explained to them what they could do (e.g., they could use calculators provided) and what they could not do (e.g., they could not communicate with each other) during the experiment sessions. After the lead author answered any questions from the participants and the consent forms were signed, the participants were led into the laboratory and were seated in front of their designated computers

We gave each participant a copy of the experimental instruction for the first treatment (see Appendix I) before the experiment, and they have ten minutes to read it. After everyone finished reading the instructions, the lead author gave an oral presentation with PowerPoint slides which emphasized the underlying uncertainty in some treatments. The participants also were given several numerical examples and were explained how their management choices could affect their profit, the ambient tax, and ambient pollution. The first author answered any questions they might have after the presentation. To make sure the participants understood the general instructions, they were required to answer a list of verifying questions correctly using the computers before they proceeded to make any choices in the experiment. The software, z-Tree, checked the participants' answers automatically and informed the participants which question(s) they did not correctly 
answer. The experiment began after everyone had correct answers. The lead author explained the answers further if some participants were still confused by any questions.

Each participant received one pair of treatments, and we implemented one pair of treatments during one session of the experiment. Uncertainty levels were kept the same in each pair of treatments but the levels of information disclosure varied. We gave new instructions and a new presentation before the second treatment. Each treatment consisted of 45 decisions rounds. The participants made one management choice and learned the level of ambient pollution, the tax that the environmental regulator imposed and their total profit from the round. We disclose the participants' choices to all their group members in treatments with information disclosure. Rounds were independent of each other because the management choices and ambient pollution did not affect any outcomes in future rounds.

After two treatments in each session, we had non-binding discussion segments. At the beginning of the discussion segments, all the participants played ten rounds of the experiment in which the tax was Pigovian-type, and the tax they received was based on their pollution levels as if the environmental regulator could monitor their emissions. We counted the profits in the ten rounds into their real earnings. After playing the ten rounds of the experiment, they were free to ask any questions and gave any comments about the experiment. We provided pizza and beverage for the discussion session.

Each session took about one hour and thirty minutes. The software calculated the final earning based on 100 rounds of the experiment, the show-up fee, and exchange rate. We paid the participants in cash at the end of the sessions. The average payoff was $\$ 22.10$ with a standard deviation of $\$ 3.80$. 
In each round, the participants chose from six management options. Their decisions directly determined their private payoff, as shown in Table 2.3. However, their emission levels were determined by their decisions and uncertainty variables. We assumed that the total emission equals the summation of six individual emissions in one group. The ambient tax for the participants is determined by the total emission and tax function in Table 2.2. For instance, if in one round each participant in one group chooses option \#2 and each has an individual emission of 2.5 after the stochastic process, the total emissions will be 15 . Everyone in the group will pay a tax that is equal to 450 experimental dollars. Everyone's total earnings in this round will be $800-450=350$ experimental dollars.

\subsection{Theoretical predictions and hypotheses}

Since the participants are not allowed to communicate with each other during the experiment, we use non-cooperative game theory to forecast the outcomes. However, we do not rule out the possibility that the participants may cooperate with each other during the experiment since the experiments are a set of repeated games (the participants may figure out that they benefit if everyone cooperates), and information of other players' actions are provided in half of the rounds. Also, we assume the participants are rational and risk-neutral when predicting some uncertainty treatments. The predicted outcomes are presented in Table 2.4.

The predicted outcomes suggest that if the participants choose not to cooperate with each other, they select management option \#3 to maximize their profit in each treatment, and the (expected) total emissions equal eighteen. Option \#3 is a Nash-equilibrium selection in this case. We also parameterize the experiment such that the Nash-equilibrium 
selections are the social optimum. However, if the participants find a way to cooperate with each other, they choose management option \#1 to maximize their profit. Thus, the expected total emissions are six in each treatment. According to previous literature, however, cooperative cases are very rare in a series of experiments investigating the ambient-based policy, except under cheap talk or communication treatments. Thus, when we outline hypotheses to test, we assume that the participants in the experiment are noncooperative with each other.

Based on the experimental design and the assumptions we make, we form three basic hypotheses related to the impact of the ambient tax policy, uncertainty, and information disclosure on social efficiency and individual behavior. The hypotheses are in the form of the null hypotheses, except Hypothesis 1.

Hypothesis 1: The ambient tax policy increases social efficiency.

Hypothesis 1 stems from the fact that that ambient tax is a disincentive for one unit of total emission increase. One unit of total emissions increase reduces each participant's payoff by 30 experimental dollars. Thus we expect ambient tax can increase social efficiency since the social efficiency is very low if no such a policy is implemented in the experiments that many researchers conducted in previous literature.

Hypothesis 2: Uncertainty levels and information disclosure do not affect the total emissions and the efficiency of the ambient tax policy.

This hypothesis emphasizes possible differences at the group level. We parameterize the uncertainty treatments such that the expected emission levels equal the emission levels under certainty treatments. Based on the risk-neutral and rational assumptions and compliance results at the group level from previous literature, we 
hypothesize that the total emissions do not change due to uncertainty treatments and the efficiency does not change either. Also, we assume Nash equilibrium behavior dominates other strategies even though information disclosure can induce behavior change under some game theory literature.

Hypothesis 3: Uncertainty levels and information disclosure do not induce behavior differences under the ambient tax policy.

Hypothesis 3 tests potential behavior differences at the individual level. Similar to the reasons for Hypothesis 2, we do not expect that behavior differences are statistically significant when presenting uncertainty and information about other players' actions.

\subsection{Results}

We highlight three key results from the experiment in this section, organized by the three hypotheses.

Result 1: The ambient tax policy increases the social efficiency.

We calculate the social efficiency from the view of the social planner. When there is no policy implemented in the experiment, we assume that there is no social efficiency at according to the results of many previous experiments and every participant select the management option \#6 as the status quo choice. We follow Spraggon [2002] to define social efficiency as "the change in the value of net social benefits as a percentage of the optimal change in social benefits from the status quo," and the mathematic expression is:

$$
\mathrm{SE}=\left(\mathrm{NSB}_{\text {actual }}-\mathrm{NSB}_{\text {status quo }}\right) /\left(\mathrm{NSB}_{\text {optimal }}-\mathrm{NSB}_{\text {status quo }}\right)
$$

where SE is the social efficiency, $\mathrm{NSB}_{\text {actual }}$ represents the actual net social benefit in each treatment and round, $\mathrm{NSB}_{\text {status quo }}$ is the net social benefit when the participants made 
choices under the condition that no policy is implemented (in our experiment, we assume this status quo choice is management option \#6 with the emission level 6), and NSB optimal represents the net social benefit with socially optimal behavior (Every participant choose management option \#3 in our experiment).

The first result comes from a simple inspection of the mean social efficiency of each treatment with the ambient based tax shown in Table 2.5 (Column 1). The average social efficiencies of all six treatments are between $63 \%$ and $69 \%$. Though there is no direct comparison group in our experiment, previous experiments, such as Suter et al. [2008] and Miao et al. [2016], the social efficiency is nearly $0 \%$ under no policy scenario. Efficiency levels of all the treatments are statistically different from $0 \%$.

Result 2a: Under the ambient tax policy, environmental uncertainty at the individual level increases total emissions; information disclosure decreases total emissions in certainty treatments.

We can observe the impact of the individual uncertainty and information disclosure on emissions in the first column in Table 2.5. Average total emissions of Treatments A and D (certainty treatments) is lower than the predicted emission. In contrast, average total (expected) emissions of Treatment B, C, E and F (uncertainty treatments) is higher than the predicted emissions. However, the effect of information disclosure is not systematic. When there is no environmental uncertainty, information disclosure decreases total emissions, but the effect does not hold when the payoff is noisy.

We use a panel data approach with a random effect to formally test the effect of individual uncertainty and information disclosure. We specify the econometric model as

$$
x_{g t}=\sum_{\mathrm{T}=\mathrm{A}}^{\mathrm{F}} \beta_{\mathrm{T}} \mathrm{T}_{g t}+v_{g}+\varepsilon_{g t}
$$


where $x_{g t}$ is the total emission of group $g$ in round $t, \mathrm{~T}_{g t}$ are the treatment dummies, $\beta_{\mathrm{T}}$ are the coefficients of each treatment, $v_{g}$ is the group specific error term and $\varepsilon_{g t}$ is the standard error over group and period.

Based on a post regression analysis, all the total emissions are different from eighteen which is the predicted pollution level and the social optimum. However, systematic differences exist between uncertainty and certainty treatments. The average total emissions of certainty treatments are statistically significantly below the social optimum. It seems a portion of people tried to cooperate with their group member to reduce their tax burden during the experiment by choosing management option \#1 and \#2. The average expected total emissions of uncertainty treatments are statistically significantly above the social optimum. We can see that the participants in uncertainty chose management options which induced expected emissions levels greater than three more often than the participants in certainty treatments. Thus the uncertainty treatments systematically induce higher emission levels than certainty treatment. Worth to mention, increasing the level of uncertainty does not change the overall emissions statistically significantly.

We cannot find systematic differences between treatments with or without perfect information of other group members' past actions by eyeball-testing of the mean total emissions of all treatments (Table 2.5, column 1). However, from the above econometric model, we find that information disclosure induces statistically significant low emissions in certainty treatments, but not in uncertainty treatments. In another word, in an environment without uncertainty, the participants tend to be more cooperative when they can observe other group members' past actions. However, when the environment is 
uncertain, or the payoffs are noisy, information disclosure does not increase cooperative behavior.

Taken together, the environmental uncertainty at the individual level increases total emissions, compared with the treatments without environmental uncertainty. The ability to observe other group members' past actions have a great impact on the total emissions when no environmental uncertainty exists.

Result 2b: Under the ambient tax policy, environmental uncertainty at the individual level does not affect the overall social efficiency, however, increasing the level of uncertainty statistically significantly reduces the allocative efficiency; Information disclosure reduces overall social efficiency.

In this part, we test the impact of uncertainty and information disclosure on social efficiency (SE) together with its decompositions: allocative efficiency (AE) and emission efficiency (EE). The decomposition follows Suter et al. [2008] and the product of AE and EE equals the SE. A low AE indicates that there is an imperfect allocation of individual emissions. A low EE means the aggregate emissions deviate far from the optimal level. AE is equal to $100 \%$ if individual emissions are perfectly allocated. In the setting of this experiment, $\mathrm{AE}$ is $100 \%$ if all group members' choices are symmetric. EE is equal to $100 \%$ if overall emissions are equal to eighteen.

We specify the following random effect models to test the effect of uncertainty and information disclosure:

$$
\begin{gathered}
\mathrm{SE}_{g t}\left(\mathrm{AE}_{g t}, \mathrm{EE}_{g t}\right)=\alpha+\sum_{\mathrm{U}=1}^{3}\left(\gamma_{\mathrm{U}} * \text { Uncertainty }_{g t}^{\mathrm{U}}\right)+\sum_{\mathrm{Info}=1}^{2}\left(\theta_{\mathrm{Info}} *\right. \\
\text { Information } \left._{g t}^{\text {Info }}\right)+\sum_{U=1}^{3} \sum_{\text {Info=1 }}^{2}\left(\psi_{\mathrm{U}-\text { Info }} * \text { Uncertainty }_{g t}^{\mathrm{U}} * \text { Information }_{g t}^{\text {Info }}\right)+v_{g}+ \\
\varepsilon_{g t}
\end{gathered}
$$


where $\mathrm{SE}_{\mathrm{g} t}\left(\left(\mathrm{AE}_{g t}, \mathrm{EE}_{g t}\right)\right.$ is the social efficiency (the allocative efficiency, the emission efficiency) of group $g$ in round $t$, Uncertainty ${ }_{g t}^{\mathrm{U}}$ are the uncertainty dummies of group $g$ in round $t$ ( 1 represents certainty, 2 represents low uncertainty and 3 represents high uncertainty) Information ${ }_{g t}^{\text {Info }}$ are the information dummies of group $g$ in round $t$ (1 represents no information, and 2 represents with information), $\gamma_{\mathrm{U}}, \theta_{\mathrm{Info}}$, and $\psi_{\mathrm{U}-\mathrm{Info}}$ are the coefficients of each dummy variable, $v_{g}$ is the group specific error term and $\varepsilon_{g t}$ is the standard error over group and period.

We show the results of the above models in Table 2.6. As expected, the first column indicates that the environmental uncertainty at the individual level does not affect the overall social efficiency. However, uncertainty, especially high uncertainty, reduces the allocative efficiency, which means uncertainty encourage heterogeneous behavior among the experiment participants. The impact of information disclosure on the efficiency is different from the predicted results according to Table 2.6. The information disclosure treatment has an overall negative effect on the social efficiency, and the major source of efficiency reduction comes from the reduction of the emission efficiency. The information disclosure increases the allocative efficiency by inducing more symmetric behavior. However, it seems the total emissions deviate more from the optimal level when the participants can observe other group members' actions.

Interestingly, we find that environmental shocks, a negative payoff in round $t$, increase emissions in round $t+1$, using a similar regression model in which the total emission is the dependent variable and environmental shock is an added independent variable. In the nonbinding communication with the participants, a large portion of them indicated that they expected the shocks would not come back so that they increased their 
pollution level in the next round. Although we emphasized that rounds were independent with each other during the experiment, the participants in uncertainty treatments acted as a gamblers' in a game and had wrong beliefs such as gambler's fallacy.

Result 3: Under the ambient tax policy, environmental uncertainty at the individual level decreases over-abatement behavior and increases under-abatement behavior; information disclosure rises over-abatement behavior.

Result 1 reveals that uncertainty and information disclosure may affect the total emissions. This result looks into the possible behavior differences from individual choices. Since only six management choices are available to choose from by the experiment participants and management choices, from \#1 to \#6, induces increasing (expected) emissions, we treat the variable (individual decisions) as ordered discrete choices. We model the individual decisions with a random-effects ordered logistic model, using uncertainty dummy variables, information disclosure dummy variables and their interaction terms as the explanatory variables.

The marginal effects of uncertainty and information disclosure treatments on individual choices are presented in Table 2.7. The estimation results (Row 1 and 2, Table 2.7) suggest that environmental uncertainty at the individual level, no matter it is at the low or high uncertainty level, statistically significantly reduces the over-abetment choices (management option \#1 and \#2) and increases the under-abatement choices (management option \#4, \#5 and \#6). It seems the participants' choices are transferred from one type of sub-optimal choices to the other type of sub-optimal choices when uncertainty treatments are imposed in the experiment. We did not find systematic choices differences between two uncertainty levels. 
Also, we find that information disclosure increases the possibilities of overabatement choices (management option \#1 and \#2). However, information disclosure does not affect the Nash-equilibrium and under-abatement selections (Table 2.7, row 3). Taken together, when no environmental uncertainty exists, and the participants can observe other group members choices, the participants are more likely to choose to over abate which indicates that they try to cooperate with each other even their choices are not the Nashequilibrium selection.

We further compare the individual choices during the experiment with the choices in the post-experiment discussion in which the participants face a Pigovian-type tax. We find that under-abatement choices significantly decrease in the uncertainty treatments. Based on the discussion with the participants, the seemingly risk-seeking behavior in the uncertainty treatment may come from the effect of uncertainty and also the feature of the group incentive (ambient tax policy).

\subsection{Conclusion}

In this study, we use an NPS water pollution economic experiment to investigate the impact of environmental uncertainty at the individual level on social efficiency and NPS polluters' behavior under the ambient tax policy. This type of environmental uncertainty imposes an uncertain relationship between individual pollution emission and individual action. The results of our experiment show that the environmental uncertainty induces different patterns of NPS polluters' behavior. Specifically, the environmental uncertainty largely decreases the possibility of collaboration among NPS polluters in noncooperative settings and increases total emission levels. As the uncertainty level increases, NPS polluters' management options vary. Compared with the behavior in an individual tax 
setting, NPS polluters are more risk-seeking under the ambient tax policy. We do not find the environmental uncertainty has a significant impact on social efficiency, however.

We allow the participants to have the information about the actions of their group member in half of our treatments in the experiment. We find that the information disclosure treatment induces a lower social efficiency, compared with no-information disclosure groups. The loss of social efficiency mostly comes from the trend of collusive behavior. The trend is triggered by simply observing other group members' action, even without any communication.

The results offer the environmental regulators some insight into the possible implementation of ambient-based policies to regulate NPS pollution. To increase the efficiency of such kind of policies, they should be aware of potential collusive behavior which is a threat to the social efficiency. Also, environmental uncertainty can induce NPS polluters to deviate from the target outcomes of related ambient-based policies. Eliminating or reducing the influence of the environmental uncertainty on payoff structures is one boost to the utilization of the ambient-based policies. 


\section{Acknowledgments}

This material is based upon work supported in part by the RI Agricultural Experiment Station Hatch Regional - RIO015-W3133 and National Science Foundation EPSCoR Track-2 Cooperative Agreement IIA-1330406, Collaborative Research: North East Water Resources Network. 


\section{References}

Bereby-Meyer, Yoella, and Alvin E. Roth. 2006. "The Speed of Learning in Noisy Games: Partial Reinforcement and the Sustainability of Cooperation." American Economic Review 96 (4): 1029-42. doi:10.1257/aer.96.4.1029.

Cochard, François, Marc Willinger, and Anastasios Xepapadeas. 2005. "Efficiency of Nonpoint Source Pollution Instruments: An Experimental Study." Environmental and Resource Economics 30 (4): 393-422. doi:10.1007/s10640-004-5986-y.

Cohen, Michele, Jean-Yves Jaffray, and Tanios Said. 1987. "Experimental Comparison of Individual Behavior under Risk and under Uncertainty for Gains and for Losses." Organizational Behavior and Human Decision Processes 39 (1): 1-22. doi:10.1016/0749-5978(87)90043-4.

Elliott, Steven R. 1998. "Experiments in Decision-Making under Risk and Uncertainty: Thinking Outside the Box." Managerial and Decision Economics 19 (4-5): 23957. doi:10.1002/(SICI)1099-1468(199806/08)19:4/5<239::AIDMDE889>3.0.CO;2-F.

Faucheux, Sylvie, and Géraldine Froger. 1995. "Decision-Making under Environmental Uncertainty." Ecological Economics 15 (1): 29-42. doi:10.1016/09218009(95)00018-5.

Hansen, Lars Gårn. 1998. "A Damage Based Tax Mechanism for Regulation of NonPoint Emissions." Environmental and Resource Economics 12 (1): 99-112. doi:10.1023/A:1008222900176.

Kahneman, Daniel, and Amos Tversky. 1979. "Prospect Theory: An Analysis of Decision under Risk." Econometrica 47 (2): 263-91. doi:10.2307/1914185.

Miao, Haoran, Jacob R. Fooks, Todd Guilfoos, Kent D. Messer, Soni M. Pradhanang, Jordan F. Suter, Simona Trandafir, and Emi Uchida. 2016. "The Impact of Information on Behavior under an Ambient-Based Policy for Regulating Nonpoint Source Pollution.” Water Resources Research, April, n/a-n/a. doi:10.1002/2015WR018142.

Neumann, John Von. 1944. Theory Of Games And Economic Behavior. Princeton University Press. http://archive.org/details/theoryofgamesand030098mbp.

Poe, Gregory L., William D. Schulze, Kathleen Segerson, Jordan F. Suter, and Christian A. Vossler. 2004. "Exploring the Performance of Ambient-Based Policy Instruments When Nonpoint Source Polluters Can Cooperate.” American Journal of Agricultural Economics 86 (5): 1203-10. doi:10.1111/j.00029092.2004.00665.x.

Segerson, Kathleen. 1988. "Uncertainty and Incentives for Nonpoint Pollution Control." Journal of Environmental Economics and Management 15 (1): 87-98. doi:10.1016/0095-0696(88)90030-7.

Spraggon, John. 2002. "Exogenous Targeting Instruments as a Solution to Group Moral Hazards." Journal of Public Economics 84 (3): 427-56. doi:10.1016/S00472727(01)00088-3. . 2004. "Testing Ambient Pollution Instruments with Heterogeneous Agents." Journal of Environmental Economics and Management 48 (2): 837-56. doi:10.1016/j.jeem.2003.11.006.

Suter, Jordan F., and Christian A. Vossler. 2013. "Towards an Understanding of The 
Performance of Ambient Tax Mechanisms in The Field:Evidence from Upstate New York Dairy Farmers." American Journal of Agricultural Economics, August, aat066. doi:10.1093/ajae/aat066.

Suter, Jordan F., Christian A. Vossler, and Gregory L. Poe. 2009. “Ambient-Based Pollution Mechanisms: A Comparison of Homogeneous and Heterogeneous Groups of Emitters." Ecological Economics, Eco-efficiency: From technical optimisation to reflective sustainability analysis, 68 (6): 1883-92. doi:10.1016/j.ecolecon.2009.01.004.

Suter, Jordan F., Christian A. Vossler, Gregory L. Poe, and Kathleen Segerson. 2008. "Experiments on Damage-Based Ambient Taxes for Nonpoint Source Polluters." American Journal of Agricultural Economics 90 (1): 86-102. doi:10.1111/j.14678276.2007.01055.x.

Vaughan, M. C. H., W. B. Bowden, J. B. Shanley, A. Vermilyea, R. Sleeper, A. J. Gold, S. M. Pradhanang, et al. 2017. "High-Frequency Dissolved Organic Carbon and Nitrate Measurements Reveal Differences in Storm Hysteresis and Loading in Relation to Land Cover and Seasonality." Water Resources Research 53 (7): 5345-63. doi:10.1002/2017WR020491.

Vossler, Christian A., Gregory L. Poe, William D. Schulze, and Kathleen Segerson. 2006. "Communication and Incentive Mechanisms Based on Group Performance: An Experimental Study of Nonpoint Pollution Control." Economic Inquiry 44 (4): 599-613. doi:10.1093/ei/cbj043.

Woznicki, Sean A., and A. Pouyan Nejadhashemi. 2014. "Assessing Uncertainty in Best Management Practice Effectiveness under Future Climate Scenarios." Hydrological Processes 28 (4): 2550-66. doi:10.1002/hyp.9804.

Xepapadeas, A. P. 1992. "Environmental Policy Design and Dynamic Nonpoint-Source Pollution." Journal of Environmental Economics and Management 23 (1): 22-39. doi:10.1016/0095-0696(92)90039-Y.

Xepapadeas, Anastasios. 2011. "The Economics of Non-Point-Source Pollution.” Annual Review of Resource Economics 3 (1): 355-73. doi:10.1146/annurev-resource083110-115945.

Young, R. A., C. A. Onstad, D. D. Bosch, and W. P. Anderson. 1989. "AGNPS: A Nonpoint-Source Pollution Model for Evaluating Agricultural Watersheds." Journal of Soil and Water Conservation 44 (2): 168-73. 
Table 2.1: Treatment conditions

\begin{tabular}{|c|c|c|c|}
\hline Treatment & Label & Information about other group members' decisions & Uncertainty Level \\
\hline A & $\begin{array}{l}\text { No information, } \\
\text { Certainty }\end{array}$ & No & Certainty \\
\hline $\mathrm{B}$ & $\begin{array}{l}\text { No information, Low } \\
\text { uncertainty }\end{array}$ & No & Low uncertainty \\
\hline $\mathrm{C}$ & $\begin{array}{l}\text { No information, High } \\
\text { certainty } \\
\text { Information, Certainty }\end{array}$ & No & High uncertainty \\
\hline $\mathrm{D}$ & & Yes & Certainty \\
\hline $\begin{array}{l}\mathrm{E} \\
\mathrm{F}\end{array}$ & $\begin{array}{l}\text { Information, Low } \\
\text { uncertainty } \\
\text { Information, High } \\
\text { certainty }\end{array}$ & $\begin{array}{l}\text { Yes } \\
\text { Yes }\end{array}$ & $\begin{array}{l}\text { Low uncertainty } \\
\text { High uncertainty }\end{array}$ \\
\hline
\end{tabular}


Table 2.2: Function forms and parameter values

\begin{tabular}{ccccc}
\hline Description & Function Form & $\begin{array}{c}\text { Parameter Values } \\
\text { Certainty }\end{array}$ & $\begin{array}{c}\text { Parameter Values } \\
\text { Low uncertainty }\end{array}$ & $\begin{array}{c}\text { Parameter Values } \\
\text { High uncertainty }\end{array}$ \\
\hline $\begin{array}{c}\text { Profit function } \\
\pi\left(\mathrm{y}_{i}, \mathrm{a}_{i}\right)\end{array}$ & $p^{*} y_{i}-\left(\mathrm{A} y_{i}{ }^{2}+\mathrm{B} a_{i}{ }^{2}\right)+\mathrm{C}$ & $\begin{array}{c}p=6.00, \mathrm{~A}=0.02, \mathrm{~B}= \\
0.05, \mathrm{C}=350\end{array}$ & $\begin{array}{c}p=6, \mathrm{~A}=0.02, \mathrm{~B}= \\
0.05, \mathrm{C}=350\end{array}$ & $\begin{array}{c}p=6, \mathrm{~A}=0.02, \mathrm{~B}=0.05, \\
\mathrm{C}=350\end{array}$ \\
$\begin{array}{c}\text { Emission Function } \\
\mathrm{r}_{i}\left(\mathrm{y}_{i}, \mathrm{a}_{i}, \varepsilon\right)\end{array}$ & $\left(\mathrm{E} y_{i}{ }^{2}-\mathrm{F} a_{i}{ }^{0.5}\right)^{*} \varepsilon /\left(\mu+\sigma^{2} / 2\right)$ & $\mathrm{E}=1 / 3750, \mathrm{~F}=4 / 75, \varepsilon$ & $\mathrm{E}=1 / 2750, \mathrm{~F}=2 / 275, \varepsilon$ & $\mathrm{E}=1 / 2750, \mathrm{~F}=2 / 275, \varepsilon$ \\
$\sim$ & $=\mu+\sigma^{2} / 2$ & $\log N(0.875,1)$ \\
$\begin{array}{c}\text { Ambient pollution } \\
\text { function } \mathrm{x}(\mathrm{r})\end{array}$ & $\sum_{i=1}^{\mathrm{I}} r_{i}$ & $\mathrm{I}=6$ & $\mathrm{I}=6$ & $\mathrm{I}=6$ \\
$\begin{array}{c}\text { Damage function } \\
\mathrm{D}(\mathrm{x})\end{array}$ & $\mathrm{H}{ }^{*} \mathrm{x}$ & $\mathrm{H}=30$ & $\mathrm{H}=30$ & $\mathrm{H}=30$ \\
$\begin{array}{c}\text { Tax function } \\
\mathrm{T}(\mathrm{x})\end{array}$ & $\mathrm{t} * \mathrm{x}$ & $\mathrm{t}=30$ & $\mathrm{t}=30$ & $\mathrm{t}=30$ \\
\hline
\end{tabular}


Table 2.3: Profit-maximizing values of production and abatement for (expected) emission levels

\begin{tabular}{ccccc}
\hline & & & & \\
Management Option & (Expected) Emissions (r) & Abatement (a) & Production $(\mathrm{y})$ & 150.00 \\
Profit $(\pi)$ \\
$\#$ \#5 & 6.00 & 0.00 & 137.81 & 800.00 \\
$\# 4$ & 5.00 & 1.46 & 123.78 & 796.92 \\
$\# 3$ & 4.00 & 2.62 & 107.91 & 785.92 \\
$\# 2$ & 3.00 & 3.93 & 89.32 & 763.81 \\
$\# 1$ & 2.00 & 5.69 & 65.87 & 724.73 \\
\end{tabular}


Table 2.4: Predicted outcomes of group emissions

\begin{tabular}{|c|c|c|c|}
\hline Treatment & Label & $\begin{array}{l}\text { Predicted (expected) total emissions } \\
\text { Under non-cooperative game theory }\end{array}$ & $\begin{array}{l}\text { Predicted (expected) total emissions } \\
\text { Under cooperative game theory }\end{array}$ \\
\hline & No information, Certainty & & \\
\hline $\mathrm{A}$ & & 18.00 & 6.00 \\
\hline B & $\begin{array}{l}\text { No information, Low } \\
\text { uncertainty }\end{array}$ & 18.00 & 6.00 \\
\hline $\mathrm{C}$ & $\begin{array}{l}\text { No information, High } \\
\text { certainty } \\
\text { Information, Certainty }\end{array}$ & 18.00 & 6.00 \\
\hline $\mathrm{D}$ & & 18.00 & 6.00 \\
\hline $\mathrm{E}$ & $\begin{array}{l}\text { Information, Low } \\
\text { uncertainty } \\
\text { Information, High }\end{array}$ & 18.00 & 6.00 \\
\hline $\mathrm{F}$ & certainty & 18.00 & 6.00 \\
\hline
\end{tabular}


Table 2.5: Average expected efficiency of treatments

\begin{tabular}{|c|c|c|c|c|c|}
\hline Treatment & Label & $\begin{array}{l}\text { Expected } \\
\text { Total } \\
\text { Emission }\end{array}$ & $\begin{array}{l}\text { Expected } \\
\text { Social } \\
\text { Efficiency }\end{array}$ & $\begin{array}{l}\text { Expected } \\
\text { Emission } \\
\text { Efficiency }\end{array}$ & $\begin{array}{l}\text { Expected } \\
\text { Allocative } \\
\text { Efficiency }\end{array}$ \\
\hline A & $\begin{array}{c}\text { No information, } \\
\text { certainty }\end{array}$ & $\begin{array}{l}16.82 \\
(3.88)\end{array}$ & $\begin{array}{c}68.34 \% \\
(0.16)\end{array}$ & $\begin{array}{c}89.65 \% \\
(0.11)\end{array}$ & $\begin{array}{c}76.60 \% \\
(0.16)\end{array}$ \\
\hline B & $\begin{array}{l}\text { No information, } \\
\text { Low uncertainty }\end{array}$ & $\begin{array}{l}19.03 \\
(3.30)\end{array}$ & $\begin{array}{c}68.99 \% \\
(0.16)\end{array}$ & $\begin{array}{c}92.56 \% \\
(0.07)\end{array}$ & $\begin{array}{c}74.51 \% \\
(0.16)\end{array}$ \\
\hline $\mathrm{C}$ & High uncertainty & $\begin{array}{l}18.59 \\
(3.30)\end{array}$ & $\begin{array}{c}62.97 \% \\
(0.16)\end{array}$ & $\begin{array}{c}92.57 \% \\
(0.07)\end{array}$ & $\begin{array}{c}68.09 \% \\
(0.17)\end{array}$ \\
\hline $\mathrm{D}$ & $\begin{array}{l}\text { Information, } \\
\text { Certainty }\end{array}$ & $\begin{array}{l}15.97 \\
(4.17)\end{array}$ & $\begin{array}{c}66.83 \% \\
(0.18)\end{array}$ & $\begin{array}{c}86.20 \% \\
(0.16)\end{array}$ & $\begin{array}{c}78.40 \% \\
(0.17)\end{array}$ \\
\hline $\mathrm{E}$ & $\begin{array}{l}\text { Information, } \\
\text { Low uncertainty }\end{array}$ & $\begin{array}{l}18.93 \\
(4.27)\end{array}$ & $\begin{array}{c}66.68 \% \\
(0.18)\end{array}$ & $\begin{array}{c}89.71 \% \\
(0.11)\end{array}$ & $\begin{array}{c}74.45 \% \\
(0.18)\end{array}$ \\
\hline $\mathrm{F}$ & $\begin{array}{l}\text { Information, } \\
\text { High uncertainty }\end{array}$ & $\begin{array}{l}19.02 \\
(3.67)\end{array}$ & $\begin{array}{c}63.99 \% \\
(0.15)\end{array}$ & $\begin{array}{c}91.58 \% \\
(0.09)\end{array}$ & $\begin{array}{c}69.99 \% \\
(0.18)\end{array}$ \\
\hline
\end{tabular}


Table 2.6: Effects of uncertainty level and information on efficiency

\begin{tabular}{|c|c|c|c|}
\hline \multirow{4}{*}{ VARIABLES } & (1) & (2) & (3) \\
\hline & Expected & Expected & Expected \\
\hline & Social & Allocative & Emission \\
\hline & Efficiency & Efficiency & Efficiency \\
\hline \multirow{2}{*}{ Low uncertainty } & 0.007 & -0.021 & 0.030 \\
\hline & $(0.039)$ & $(0.035)$ & $(0.020)$ \\
\hline \multirow[t]{2}{*}{ High uncertainty } & -0.054 & $-0.085 * *$ & 0.029 \\
\hline & $(0.039)$ & $(0.035)$ & $(0.020)$ \\
\hline \multirow{2}{*}{ Information } & $-0.015^{* *}$ & $0.018 * *$ & $-0.035 * * *$ \\
\hline & $(0.008)$ & $(0.008)$ & $(0.006)$ \\
\hline \multirow{2}{*}{ Low uncertainty \#Information } & -0.008 & $-0.019 *$ & 0.006 \\
\hline & $(0.011)$ & $(0.011)$ & $(0.008)$ \\
\hline \multirow{2}{*}{ High uncertainty \# Information } & $0.025 * *$ & 0.001 & $0.025^{* * *}$ \\
\hline & $(0.011)$ & $(0.011)$ & $(0.008)$ \\
\hline Observations & 3,780 & 3,780 & 3,780 \\
\hline
\end{tabular}

Standard errors in parentheses. $* * * \mathrm{p}<0.01, * * \mathrm{p}<0.05, * \mathrm{p}<0.1$ 
Table 2.7: Marginal effects of uncertainty and information disclosure based on the random-effects ordered logistic model

\begin{tabular}{|c|c|c|c|c|c|c|}
\hline VARIABLES & $\begin{array}{c}\text { Management } \\
\text { Option } \\
\# 1 \\
\end{array}$ & $\begin{array}{c}\text { Management } \\
\text { Option } \\
\# 2 \\
\end{array}$ & $\begin{array}{c}\text { Management } \\
\text { Option } \\
\# 3 \\
\end{array}$ & $\begin{array}{c}\text { Management } \\
\text { Option } \\
\# 4 \\
\end{array}$ & $\begin{array}{c}\text { Management } \\
\text { Option } \\
\text { \# 5 } \\
\end{array}$ & $\begin{array}{c}\text { Management } \\
\text { Option } \\
\# 6 \\
\end{array}$ \\
\hline Low uncertainty & $\begin{array}{c}-0.0681 * * * \\
(0.024)\end{array}$ & $\begin{array}{c}-0.127 * * * \\
(0.040)\end{array}$ & $\begin{array}{c}0.052 * * \\
(0.022)\end{array}$ & $\begin{array}{c}0.076 * * * \\
(0.025)\end{array}$ & $\begin{array}{c}0.037 * * * \\
(0.012)\end{array}$ & $\begin{array}{c}0.031 * * * \\
(0.011)\end{array}$ \\
\hline High uncertainty & $\begin{array}{c}-0.057 * * * \\
(0.025)\end{array}$ & $\begin{array}{c}-0.0289 * * \\
(0.00848)\end{array}$ & $\begin{array}{c}0.049 * * \\
(0.022)\end{array}$ & $\begin{array}{c}0.058 * * \\
(0.024)\end{array}$ & $\begin{array}{c}0.027 * * \\
(0.012)\end{array}$ & $\begin{array}{c}0.021 * * \\
(0.009)\end{array}$ \\
\hline Information & $\begin{array}{c}0.007 * * * \\
(0.002)\end{array}$ & $\begin{array}{l}0.007 * \\
(0.004)\end{array}$ & $\begin{array}{c}-0.008 * * * \\
(0.003)\end{array}$ & $\begin{array}{c}0.004 \\
(0.003)\end{array}$ & $\begin{array}{l}-0.001 \\
(0.001)\end{array}$ & $\begin{array}{l}-0.001 \\
(0.011)\end{array}$ \\
\hline
\end{tabular}

$N=22,680$; Delta-method standard errors in parentheses. $* * * \mathrm{p}<0.01, * * \mathrm{p}<0.05, * \mathrm{p}<0.1$ 


\title{
MANUSCRIPT-3
}

\section{Experimental evidence of learning effects under an ambient tax policy}

\section{(To be submitted)}

\author{
by \\ Haoran Miao ${ }^{\text {a }}$, Todd Guilfoos ${ }^{\mathrm{a}}$, and Emi Uchida ${ }^{\mathrm{a}}$ \\ ${ }^{a}$ Department of Environmental and Natural Resource Economics, University of Rhode Island, Kinston, RI
}

\begin{abstract}
In studies using laboratory experiments to investigate the ambient-based policies, the participants adapt their strategy after many rounds of interaction in some circumstances. We conduct a laboratory economic experiment to test how nonpoint source polluters' behavior evolves under different scenarios. We apply the experiment data to the experience weighted learning model and find that the participants are mainly reinforced to adapt their behavior, and their learning processes vary according to different levels of environmental uncertainty. Specifically, the ability to play a strategy corresponding to the most attractive payoffs in previous rounds diminishes as the uncertainty level increases. Information disclosure neutralizes this effect. We then develop an agent-based model to scale up the findings from the experiment. We calibrate the agents' learning abilities with the parameters estimated from the experience weighted learning model learning model. From the simulation results, we find that environmental shocks decrease emission level in the $\mathrm{ABM}$ after many rounds of interactions given that we disclose group members' actions, but we do not observe this effect in the laboratory experiments.
\end{abstract}

JEL Classification: $C 91, Q 52, Q 53, D 01, C 63$ 
Keywords: Nonpoint source pollution, environmental uncertainty, information disclosure, laboratory experiment, and agent-based modeling 


\subsection{Introduction}

In the past two decades, researchers have frequently used controlled laboratory experiments to test the efficiency and effectiveness of those proposed policies to reduce NPS pollution. For instance, Cason et al. [2003] conducted a laboratory experiment of a sealed-offer auction to seek voluntary contribution to control NPS pollution and found that revealing the high environmental benefits to the potential sellers induced misrepresentative offers. The experimental results suggested that concealing the information of ecological benefits may improve the policy efficiency. Taylor et al. [2004] used a similar experimental auction method to study nonpoint source polluters' voluntary emission-reduction behavior under a group contract. They found that the group contract could be efficient and effective to control NPS pollution, but with limitations. The ambient-based policies proposed by Segerson [1988] represented another promising type of policies and a series of laboratory experiments were conducted to measure their advantages and disadvantages. Spraggon [2002] tested the effectiveness of different ambient-based policies and found that the ambient tax and the combination of ambient subsidy and tax may work better than others. Cochard, Willinger, and Xepapadeas [2005] had slightly different results in their laboratory experiments, compared to Spraggon [2002]. Economists also investigated the effects of heterogeneous NPS polluters [e.g., Spraggon, 2004; Suter, Vossler, and Poe, 2009], communication [e.g., Vossler et al., 2006], and threshold differences [Suter et al., 2008] under ambient-based policies using laboratory experiments.

Despite the growing issue on this topic, it is not well understood how players adapt or evolve toward any equilibrium. In experimental studies, researchers typically model NPS pollution problems as finite repeated normal-form games with multiple players and assume 
that players' behavior stabilizes in a short period. They use non-cooperative or cooperative game theory to predict players' behavior in each independent round. In most of the experimental designs, Nash equilibria are socially desired abatement strategies, and the empirical analyses focus on the average treatment effects of ambient-based policies across all rounds under different scenarios. Moreover, a few previous studies find that the players in the repeated games with NPS pollution settings may not form beliefs which are best strategies for them and stabilize their behavior in the short term. For example, Spraggon [2002] shows that players' still change their emission levels after more than twenty rounds, even in the promising ambient tax/subsidy and tax instruments. Suter, Vossler, and Poe [2009] demonstrate that total emission levels gradually converge to social optimum in about twenty rounds. Moreover, Miao et al. [2016] use a laboratory economic experiment in a setting with realistic in-stream nutrient transport dynamics to test the effectiveness of the ambient tax/subsidy policies. They show that the observed social efficiency increase over time in treatments with more frequent information on pollution over time. Also, Miao et al. [2017] observe unstable social efficiency after forty rounds of the experiment in all the treatments. The behavior does not converge to the Nash equilibrium despite long-term interactions. All the evidence suggests that players in the game with NPS pollution settings adapt their strategies over time instead of reasoning their ways, forming their beliefs or finding their best strategy in a short period. This study aims to fill these literature gaps by understanding how players learn to adapt or evolve toward any equilibrium in the repeated non-cooperative game with NPS pollution settings.

Our study also aims to test whether or not adding environmental uncertainty to the games with ambient-based policies decreases players' learning ability. Learning patterns 
in games with strategic uncertainty is different from the patterns in individual-decision making because the games in which each player gains experience contains other players who adapt their behavior as they learn experience [Erev and Roth, 1998]. The strategic uncertainty, which means one individual does not have knowledge about other players' action, is not the only type of uncertainty in the NPS pollution problems. Environmental uncertainty, which is independent of players' actions, represents one important feature of NPS pollution. It could come from a variation in temperature, rainfall, or other stochastic processes [Yong et al., 1998] in the NPS water pollution problems. The second motivation for the study is that we do not understand learning patterns well in the NPS pollution settings under ambient-based policies, especially when the settings combine strategic uncertainty and environmental uncertainty. Bereby-Meyer and Roth [2006] show that noisy payoff decreases players' ability to learn to cooperate in the repeated prisoner's dilemma. Similar to this study,

One important design in most experiments studying ambient-based policies is that individual player only knows the ambient pollution level and its payoffs after one round and other players' actions and their payoffs are hidden from the individual. Such design assumes that NPS polluters cannot observe other group members' actions. We vary this assumption as a dimension of treatments to mimic an extreme scenario in the field that nonpoint source polluters' actions are hidden from the environmental regulator but perfectly observed by group members. As a few studies on public goods games [e.g., Dong, Zhang, and Tao, 2016] point out that the dynamics of one player' behavior can be explained partly by other group members' behavior, we hypothesize that knowing other players' action after one round of game can change players' learning patterns. 
Based on these motivations, we conduct a laboratory experiment with implementation of an ambient tax policy and a repeated non-cooperative game to achieve the goals that we mentioned above. We adopt the classic NPS source pollution setting from [Segerson, 1988] and vary the environmental uncertainty at the individual level, including certainty, low uncertainty, and high uncertainty. Also, we disclose group members' actions to one-half of the sessions of the experiments. Furthermore, we use the most extended interaction rounds (45 rounds) among the studies investigating the policies to reduce NPS pollution to check the learning behavior over a long time horizon.

The core research question in this study is whether subjects form beliefs over time or their decisions are reinforced by previous payoffs under uncertainty. To test this question, we utilize the experience weighted attraction (EWA) learning model [Camerer, and Ho, 1999]. EWA is an appealing model for this study to discover how the subjects adapt their strategies with an ambient-based policy because it integrates belief-based models and reinforcement learning. We then use post-estimation techniques to compare learning patterns across diffident environmental uncertainty levels and information set.

As the final analysis, we take the parameters from the learning model to calibrate an agent-based model (ABM) to scale up and complement the findings from the laboratory experiments. The application of an $\mathrm{ABM}$ is motivated by fundamental limitations of laboratory experiments that experimenters are typically limited in the numbers of subjects that can be recruited and the interaction rounds. One may question that different behavior patterns may appear if we implement the experiment at a large scale and for an extended time horizon. Duffy [2006] argues that agent-based models (ABMs) are good complements of human subjects experiment and cheap computer power of ABMs can overcome the 
experimenters' limitations. Agents in the ABMs are analogous to the subjects in the laboratory experiments. They are autonomous, and they may have memory and learning abilities. They can interact with the other agents and environment that the experimenters build up in the model. We then calibrate an ABM with agents calibrated by parameters estimated from the EWA learning model to scale up findings from the laboratory experiment. We ensure that the agents are autonomous and heterogeneous and they can learn to adapt their strategies. In a nutshell, one agent in the $\mathrm{ABM}$ mimics one subject in the experiment. After building up the ABM, we first compare the simulation results with the experimental data to ensure the credibility of the ABM. We then increase the number of agents in the $\mathrm{ABM}$ and the number of interaction periods. We also test the sensitivity of the ambient tax policy when varying the number of players in one group.

From the estimation results of the learning model, we find that players usually do not form beliefs in the game with an NPS pollution setting which means most players do not have beliefs about what other players will do. They, in general, use reinforcement learning to adapt their strategies. That is, they do not care about the hypothetical payoffs of unchosen strategies. The players' abilities to map attractions into choice probabilities decreases as the environmental uncertainty level increases. Providing lagged information about other players' behavior significantly increases the abilities to map attractions into choice probabilities in the low and high environmental uncertainty treatments. Based on the simulation results of the $\mathrm{ABM}$, different behavior pattern may show up under certain circumstances when we scale up the number of NPS polluters and interaction times. We suggest that the agent-based modeling techniques can be used to double check the answers we have from the laboratory experiments. 
In the next section, we present the details of the experiment. In Section 3.3 we report the results from the learning model, while in Section 3.4 we state the simulation results of the ABM. Section 3.5 provides a summary and a discussion of some extensions of this study.

\subsection{Experimental design, Parameterization, and Protocol}

The experiment we use to explore the players' learning process in the NPS pollution settings under ambient-based policies in this manuscript is identical to the one we conduct in Miao et al. [2017]. However, because we are particularly interested the learning behavior, and there is potential spill-over effect in the second part of the sessions of the experiment, we only use the experimental data we collect from the first part of each session. Thus, different from Miao et al. [2017], this manuscript here utilizes a pure betweensubjects experimental design.

\subsubsection{Basic design}

The basic design of the experiment is from the setting of Segerson [1988]. We denote each farm in a small watershed by $i$, and there are $I$ identical farms in total. Farm $i$ can produce output $y_{i}\left(y_{i} \geq 0\right)$ and sell it at a fixed price $p . C_{i}=C\left(y_{i}, a_{i}\right)$ represents the cost

of Farm $i$, where $a_{i}\left(a_{i} \geq 0\right)$ is abatement effort, $C_{y i}=\partial C_{i} / \partial y i>0$ and $C_{a i}=\partial C_{i} / \partial a_{i}>0$.

$r_{i}=r\left(y_{i}, a_{i}, \boldsymbol{\varepsilon}\right)$ denotes the emission function of Farm $i$ where $r_{y i}=\partial r_{i} / \partial y i>0$ and $r_{a i}=\partial$ $r_{i} / \partial a_{i}<0$ and $\varepsilon$ is a vector of stochastic variables such as whether. The function $x=x\left(r_{1}\right.$, $\left.r_{2}, \ldots, r_{I}\right) \equiv x(\boldsymbol{a}, \boldsymbol{y}, \boldsymbol{\varepsilon})$ represents the ambient pollution level of the common pool in the watershed. In the above function, $\boldsymbol{a}$ is a vector of abetment efforts of $I$ farms and $\boldsymbol{y}$ is a 
vector of production of $I$ farms. The total environmental damages of a common pool in the watershed are a function of the ambient pollution level and equal $D(x)$, where $D(0)=0$, $D^{\prime}(x)>0$ and $D^{\prime \prime}(x) \geq 0$.

We impost the following ambient tax on each farm in the small watershed to make sure all the farms abate the pollution at the rate of socially optimal level:

$$
T(x)=\left\{\begin{array}{cc}
t_{i} *(x-\bar{x}) & \text { if } x>\bar{x} \\
0 & \text { if } x \leq \bar{x}
\end{array}\right.
$$

where $t_{i}$ is equal to

$$
t_{i}=\frac{\mathrm{E}\left[D^{\prime}(x) * x_{y_{i}}\right]-\mathrm{E}\left[D^{\prime}(x) * x_{a_{i}}\right]}{\mathrm{E}\left[x_{y_{i}}\right]-\mathrm{E}\left[x_{a_{i}}\right]}
$$

Readers can find more details about the experimental design in Miao et al. [2017].

\subsubsection{Parameterization and experimental protocol}

As mentioned in the introduction, we utilize a $3 \times 2$ experimental design which generates six treatments in total (Table 3.1). We implement three uncertainty levels, which includes certainty, low uncertainty, and high uncertainty. The second treatment dimension contains no-information disclosure and information disclosure.

The first treatment dimension is essentially different in the parameterization of the stochastic vector, $\boldsymbol{\varepsilon}$, in the basic design. For simplification, we assume the vector is one dimension. In the certainty treatment, the stochastic term is equal to one. In the low uncertainty treatments, the stochastic term is a normalized (mean $=1$ ) lognormal distribution with a small variation parameter. In the high uncertainty treatments, the mean the stochastic term is still equal to one, but with a relatively high variation parameter. The second treatment dimension is different in the ability to observe group members' actions. When players are in no-information disclosure treatments, they receive information about 
the ambient pollution level and their payoffs. When players are in information disclosure treatments, they receive additional information about other players' actions.

In Table 3.2, we present the ambient pollution function, damage function, and the ambient tax function which are mostly drawn from previous literature. The threshold of ambient tax equals zero in our experiment which means we impose a tax on every unit of pollution. In the basic design, the participants of the experiment need to make two choices, the abatement level, and the production level. We use the idea from Suter et al. [2008] to simplify the participants' choices as a management option. In Table 3.3, one management option maximizes the pre-tax profit subject to a specific (expected) emission levels.

Forty-two groups participated in twenty-one experimental sessions. Most of them were URI undergraduate students. Each group had six participants. We used z-Tree [Fischbacher, 2007] to run the experiment and document all decisions made by the participants. All sessions of experiments were run at the Policy Simulation Lab of the University of Rhode Island. A copy of the experimental instruction for the first treatment (see Appendix IV) was given to the participants before the experiment. They had ten minutes to read it. After everyone finished reading the instruction, the lead author gave an oral presentation and explained the underlying uncertainty in some treatments, several numerical examples, and how their management choices affected their profit, the ambient tax, and ambient pollution. Each session took about one hour and thirty minutes. The average payoff was $\$ 22.1$ with a standard deviation of $\$ 3.8$.

Each treatment contains forty-five rounds. In each round, the participants chose one of six management options. Their decisions determined their private payoff which was shown in Table 3.3. Their emission levels were determined by their decisions and the 
stochastic variables. The total emission equals the summation of six individual emissions in one group. The total emission and tax function in Table 3.2 form the ambient tax. More details about the Parameterization and the experimental protocol can be found in Miao et al. [2017].

\subsection{The learning model and results of econometric analysis}

We use an econometric model of learning to investigate how players adapt their strategy in NPS pollution problem settings under ambient-based policies and how the adaption is different among different environmental uncertainty levels and different information sets. The experience weighted attraction (EWA) learning model develop by Camerer, and Ho [1999] are well suited for the analysis of learning process of players in repeated economic experiments. It combines reinforcement learning models and beliefbased models as a single learning model. This section describes the EWA model and presents the estimation results which offer insights about how learning differs in different treatments.

\subsubsection{The learning model}

Based on the design and parameterization in Section two, we use the following notation to describe the EWA model suited for our experimental data. Each player $i$ has $m$ pure strategies ( $m=6$ and Strategies are choices of management options in our experimental setting). Let $s_{i}^{j}$ denote that plaer $i$ play the strategy $j$, one among $m$ strategies. Since we expand the basic design of NPS polluter problem into repeated games, we use $t$ to denote one period in the game. Let $s_{i}(t)$ be the strategy of player $i$ in period $t$. At time $t$, we cluster the other players' strategies, the environmental uncertainty level together and 
denoted them as $z(t)$. If player $i$ chooses strategy $j$ in time $t$, her or his expected payoff of choosing strategy $j$ in time $t$ is denoted as $\pi_{i}\left(s_{i}^{j}, z(t)\right)$ and we assume player $i$ in time thas a numerical attraction $A_{i}^{j}(t)$. Also, we assume the numerical attraction determines the probability of choosing strategy $\mathrm{j}$ in round $\mathrm{t}+1$ by the following logistic function:

$$
P_{i}^{j}(t+1)=\frac{e^{\lambda A_{i}^{j}(t)}}{\sum_{k=1}^{m} e^{\lambda A_{i}^{k}(t)}}
$$

The new parameter $\lambda$ in equation (3) is the player $i$ 's sensitivity for mapping attractions into choice probabilities. Numerically speaking, if $\lambda$ equals a number not statistically different from zero, we know that player $i$ chooses his or her strategies randomly (in our setting, player $i$ chooses each management options at the probability of about 1/6). If $\lambda$ equals a significantly large number, we can see that player $i$ chooses the most attractive strategies more often than others.

We assume the attraction of player $i$ of choosing strategy $j$ in period $t$ is determined by the attraction in period $t-1$, and the expected payoff of choosing strategy $j$ in period $t$. The attraction for each strategy in each round of the experiment are updated based on the following equation:

$$
A_{i}^{j}(t)=\frac{\Phi N(t-1) A_{i}^{j}(t-1)+\left[\delta+(1-\delta) I F\left(s_{i}^{j}, s_{i}(t)\right)\right] \pi_{i}\left(s_{i}^{j}, z(t)\right)}{N(t)}
$$

In equation (4), $N(t)$ is a weight on the past attractions which follows the updating method: $N(t)=\Phi *(1-\kappa) * N(t-1)+1$. The binary variable function $\mathrm{I} F\left(s_{i}^{j}, s_{i}(t)\right)$ is equal to zero if strategy $j$ is not the strategy chosen by player $i$ in round $t$. Otherwise, the indicator is equal to one. The parameter is $\Phi$ a discount variable of previous attractions. If player $i$ forgets or ignores previous experience deliberately because of various reasons, for example, uncertainty environment, the value of $\Phi$ would be small. In the learning process, 
the strength of forgone payoff of strategies that player $i$ dose not choose in period $t$ is weighted by the parameter $\delta$. A small $\delta$ would indicate that subjects tend to be reinforced by previous choices. Parameter $\kappa$ is the discount rate of the experience weight $N(t)$. Variables $N(t)$ and $A_{i}^{j}(t)$ have initial value $N(0)$ and $A_{i}^{j}(0)$.

We follow previous literature [e.g., Feri et al., 2010] and use the maximum likelihood method to estimate the parameters for each treatment, and the following equation gives the likelihood function:

$$
L(\lambda, \Phi, \delta, \kappa, N(0))=\prod_{i=1}^{6}\left[\prod_{t=1}^{45} P_{i}^{s_{i}(t)}(t)\right]
$$

In equation (9), we impose the following necessary restrictions on the parameters $\lambda, \Phi, \delta, \kappa$, and $N(0): \lambda \in(0, \infty), \Phi, \delta, \kappa \in(0,1]$ and $N(0) \in\left(0, \frac{1}{1-(1-\kappa) * \Phi}\right]$ to make sure the model is identifiable. All experience weights and attractions are updated over time when estimating the parameters of the model except the initial value of $A_{i}^{j}(0)$. We estimate the initial attractions of all strategies using the first period data based on the method proposed in Ho, Wang, and Camerer [2008]. For each treatment, we use the data of 42 players (seven groups) decisions over 45 rounds to estimate the learning parameters.

\subsubsection{Estimation results of the EWA learning model}

We present the estimates for parameters $\lambda, \Phi, \delta, \kappa$, and $N(0)$ in the EWA learning model in the Tables 3.4a and 3.4b. Table 3.4a reports the parameter estimates of the treatments in which the experiment participants only know their own payoffs and ambient pollution levels after each round of the experiment. Table $3.4 \mathrm{~b}$ contains the estimation results of the treatments in which the players know additional information about the actions of all group members. 
The first parameter $\lambda$ in all treatments are all statistically significantly greater than zero, which implies that the participants of the experiment do not choose their strategies randomly in all treatments under the ambient tax policy. This result is not surprising since we can observe a non-uniform distribution of decisions in all treatments. When the participants cannot observe group members' strategies, the ability of mapping attractions into choice probabilities decreases as the uncertainty levels increases (Table 3.4a, row 1). The estimate of $\lambda$ for the treatment without individual-level environmental uncertainty (Treatment A) equals 1.72 , which is statistically significantly higher than the value of $\lambda$ (1.01) in the low uncertainty treatment (Treatment B). Hence, if the participants in the two treatments face the same attractions, the participants experiencing the low individual-level environmental uncertainty are less likely to choose the strategy with the highest attractions than the participants in the certainty treatment. By the same token, the participants in the treatment with high individual-level environmental uncertainty are less sensitive to map attractions into choice probabilities than the participants experiencing low individual-level environmental uncertainty. In general, the increasing uncertainty level induces more randomness of choosing strategies under the ambient tax policy.

When the participants can observe group members' actions, the ability to map attractions into probabilities substantially increases in the uncertainty treatments. By comparing the first rows of Table $3.4 \mathrm{a}$ and Table $3.4 \mathrm{~b}$, we can see that additional information about group members' actions does not affect the values of $\lambda$ in the certainty treatments (Treatments A and D). However, the values of $\lambda$ in uncertainty treatments with information disclosure (Treatments $\mathrm{E}$ and $\mathrm{F}$ ) are statistically significantly greater than corresponding values of $\lambda$ in uncertainty treatments without information disclosure 
(Treatments B and C). The trend that increasing uncertainty induces randomness of choices still holds. In general, we can conclude that under the ambient tax policies knowing group members' actions help the participants to allocate more probabilities to the strategies with the same attractions.

The value of $\Phi$ are all statistically significantly greater than zero, and the values are all relatively close to one, ranging from 0.88 to 0.98 , reflecting that the decay rate of previous attractions are low and the previous play has a strong influence on the attractions. The values of $\Phi$ are notably significantly larger in uncertainty treatments than in certainty treatments. It implies that under the ambient tax policy, previous experience is significant to the participants in an uncertain environment and they discard old experience more quickly in without any uncertainty. However, we find that the additional information about the group members' actions does not affect the decay rate of previous attractions (second rows of Table $3.4 \mathrm{a}$ and $3.4 \mathrm{~b})$.

We observe $\delta$ which are not statistically different from zero in all the uncertainty treatments. It indicates that the participants neglect the possible payoffs from unchosen strategies under the individual-level environmental uncertainty. Their learning type is close to reinforcement learning type, which means they are mainly reinforced by the payoffs they received during the experiments. In both certainty treatments, the values of $\delta$ are statistically significantly different from zero. It demonstrates that the participants who do not experience the environmental uncertainty at the individual level under the ambient tax policy take into account the hypothetical payoffs from unchosen strategies and put a positive weight on those strategies. In other words, they practice belief-based learning. We do not observe a significant impact of information disclosure on the weight of unchosen 
strategies since the differences of the values of $\delta$ are not statistically significant (the third row of Tables 3.4a and3.4b).

The estimates for $N(0)$ are the initial values of the weights on past attractions in the six treatments (last row, Tables 3.4a and 3.4b). It reflects the pregame experience of the participants of each treatment. Based on record of our post-experiment conversation parts, none the participants did not experience such types of experiment before. Therefore, it is not surprising that the values of $N(0)$ are very different from each other in those treatments given that we have between-subjects experimental design. Also, it makes senses that the levels of environmental uncertainty and the different sets of information disclosed to the participants have no systematic impact on the weights of past attractions.

Estimates of $\kappa(\mathrm{t})$, the discount rates of $N(t)$, however, are systematically different across treatments. When the participants cannot observe group members' actions, the discount rates increase as the levels of environmental uncertainty increases. It implies that the participants significantly update their weights on the past attractions under uncertain environment as the experiment continues. We can see from the fourth row of Table 3.4a that the values of $\kappa$ are statistically significantly different from zero in the uncertainty treatments. The magnitude of updating increases when they experience an environment with more variation. The trend also holds where the participants can observe group members' actions (fourth row, Table 3.4b). The ability to know group members' actions generally induces decreases in the discount rates (fourth row of Table 3.4a and 3.4b).

To sum up, the estimates of the EWA learning model suggest that NPS polluters update their experience about their strategies over time and they may only be reinforced previous plays and payoffs, especially in an uncertainty environment. This finding is likely 
to explain in part why behavior does not converge to a certain level as predicted after many rounds of interactions under the ambient tax policy. Environmental uncertainty degrades the NPS polluters' ability to map the attractions into probabilities, which may induce more randomness of strategy choices. This effect becomes larger as the uncertainty level increases, and it potentially induces more variation of strategy choices. Also, we found instances where the environmental uncertainty makes the NPS polluters neglect the payoffs

of the unchosen strategies. Therefore, it could be one reason that the ambient tax policy does not induce perfectly desirable behavior all the time. The information set is also important to the learning process in the experiment. Knowing group members' actions may enhance NPS polluters' abilities to map the attractions into the choice probabilities in an uncertain environment. In the next section, we integrate the estimation results of the EWA learning into an $\mathrm{ABM}$ to scale up the findings we have from the experiment.

\subsection{ABM framework, calibration, and simulation results}

In this section, we describe the framework and calibration of the ABM which is used to scale up the NPS polluters' behavior. Its simulation results are presented after the description of the ABM. We use the Python language to build up the ABM.

\subsubsection{The ABM framework and calibration}

The ABM uses the same environment as in the laboratory experiment so that we can have direct comparisons between the real experiment participants and simulated agents. We create 1200 agents and divide into 200 groups. Each group with six agents is located in an isolated watershed, and their production activities increase their profit but degrade their local water quality. Identical to the laboratory experiment, the level of 
degradation depends on their management options and their environment which is changed by the uncertainty treatment types. The information sets provided to the agent also vary across treatments. The agents in a group interact with group members for 450 rounds through the ambient tax policy. That is, one agent's choice of management options affect its own and group members' profit through the ambient tax policy.

We use the estimation results of the EWA learning model to specify agents' behavior. This specification ensures that the agents adapt their strategies over time, as well as autonomy and heterogeneity of the simulated agents. We assume the agents maximize their expected profits, weigh forgone payoffs, update old experience, be reinforced by previous actions and learn to adapt their strategies based on different environment and information sets. After processing all the information, agents update their attractions of each management options at the end of each period. They make their final choices based on a logistic function same as the choice function in the EWA learning model. In a nutshell, the management options with the highest attractions in period $t$ have the most top probabilities to be chosen by the agents in period $t+1$. Worth to mention, the static Nash equilibrium of each period in the $\mathrm{ABM}$ is unique and identical to the dominant Nash strategies in the experiment.

Fehr and Fischbacher [2003] point out the initial plays matter in public goods games. To test the sensitivity of initial choices in our setting, we initiate the ABM with three methods. One is to randomly choose arrays of management options, which means each agent choose each management options with equal probability of 1/6. The second way is to use the pre-experiment attraction data that we estimate for the EWA learning model estimation to calculate six arrays of prospects respecting to six treatments. The 
agents choose the management options independently based on the calculated probability arrays. The third method is to use specific management option combinations. For example, we try to start from the collusive equilibrium or status quo strategies. We also increase the number of group members in each group to echo the group size effect in the public goods literature [e.g., Isaac and Walker, 1988].

\subsubsection{Simulation results of the ABM}

We show part of the simulation results of the ABM in the Figure 3.3a and 3.3b. This cluster of simulation uses the second method we describe in Part 4.1 to decide the initial condition of the simulation. The Figure 3.3a exhibits the dynamic behavior of 1200 agents over 450 rounds of the treatments in which the experiment participants only know their payoffs and ambient pollution levels. Figure $3 \mathrm{~b}$ displays the evolution of all the agents' behavior when they can get additional information about the actions of all group members.

From the simulation results, we can see that the agents' strategies do not converge to a point even though there are many rounds of interactions and plenty of interacted agents, no matter what the combinations of learning parameters are. It seems that choices of the agents who are not exposed to the environmental uncertainty have less variation than choices under uncertainty. Also, when the agents know group members' actions, it appears that the additional information decreases choice variation under the environmental uncertainty. Those general findings agree with the outcomes that we find from the experiment.

We also find that the simulation results can approximately match the participant's behavior in the experiment under the ambient tax policy in the uncertainty treatments when 
there is no additional information disclosed to the participants. The average individual emission levels of the experiment participants are all slightly above the social optimum. Miao et al. (2017) show that the environmental uncertainty at the individual level induces under-abatement. The simulation results seem to support the results at the large scale and in the extended period. Also, we find the environmental uncertainty increases the variety of decisions in the ABM (Figure 3.3a). This finding also coincides with the outcome of the experiment. However, we cannot observe over-abatement behavior in the ABM when the agents do know their group members' actions, and they do not face any environmental uncertainty. We conjecture that in the collusive experiment equilibrium may play a role in the choices of management options and the participants find ways to cooperate with each other to some degree. The first assumption of the EWA learning model is that the participants play a non-cooperative game. Therefore, the agents in the ABM may lack the ability to find a way to cooperate with each other. It could be one potential caveat of using the EWA learning model to specify the agent behavior in an ABM.

When the information about group members' action is provided to the agents in the $\mathrm{ABM}$ and the participants in experiments, their behavior is similar in the uncertainty treatments in the first few periods (Figure $3.2 \mathrm{~b}$ and $3.3 \mathrm{~b}$ ). However, after a few rounds of interactions, the agents in the $\mathrm{ABM}$ form a trend to decrease their emission levels under the high level of environmental uncertainty. The choices after many rounds of interactions oscillate around the socially optimal level. With a more in-depth inspection of this behavior, we find that the large shocks, in the form of significant negative payoffs, decreases the attractions of choices with emission levels exceeding the socially optimal 
emission. We cannot observe this trend from the experiment data, which can be caused by the lack of enough interactions.

These results suggest that after many rounds of learning, for example, several hurricanes in a short period, people understand the importance of adaptation and begin the adaptation process and react to others' adaptation behavior. However, in the experiment, because of the limited time in the laboratory, the shocks in the high environmental uncertainty treatments may not build up enough negative attractions. In the postexperiment nonbinding conversation with the experiment participants after the experiment sessions, most people thought that the shocks would come back after many rounds given one shock happened, and they usually increased their emission levels after encountering any shocks. The experimental data indeed reveal this type of behavior. When the agents can observe group members' behavior, and they experience no environmental uncertainty, their behavior is close to the participants' behavior in the experiment.

We change the way we define the initial conditions of the ABM to check the sensitivities of initial choices. We find that the dynamic behavior of the agents is not sensitive to the initial decisions under the ambient tax policy. Their choice quickly converges to the range of options in the first cluster of $\mathrm{ABM}$ as reported above. The simulation results are not sensitive to the group size, either.

\subsection{Conclusion}

We run a laboratory experiment to infer how the NPS polluters adapt their strategies under the ambient tax policy and how the environmental uncertainty at the individual level and information disclosure affect their learning patterns. We find that NPS polluters may 
be mainly reinforced by previous plays under the ambient tax policy, especially they experience environmental uncertainty. This results could partially explain why equilibria cannot be reached in most economic experiments studying ambient-based policies. The increased level of environmental uncertainty decreases NPS polluters' ability to choose options with high attractions. Also, the ability to know group members' actions may help NPS polluters to make better decisions.

The simulation results of the ABM reveal that the agents calibrated by the EWA learning models can mimic subjects' behavior in the experiment. Also, the results show that the NPS polluters' behavior may not converge to socially desired behavior even in the long run under the ambient-based policy. Also, the results tell us that the shocks under high environmental uncertainty could only be effective in the long term given they can observe other group members' actions. Initial choices and group size seemingly do not affect the effectiveness of the ambient tax policy.

In the future work, we may change the environment of the ABM where the agents interact with other group members. More realistic production functions, abetment functions, damage functions and spatial information can be introduced into the environment while we keep the learning parameters of the agents unchanged. After running the $\mathrm{ABM}$, the more realistic setting can offer more external validities to test the ambient tax policy when field studies are still hard to accomplish. 


\section{Acknowledgments}

This material is based upon work supported in part by the RI Agricultural Experiment Station Hatch Regional - RI0015-W3133 and National Science Foundation EPSCoR Track-2 Cooperative Agreement IIA-1330406, Collaborative Research: North East Water Resources Network. 


\section{References}

Barr, Jason, and Troy Tassier. 2010. "Endogenous Neighborhood Selection and the Attainment of Cooperation in a Spatial Prisoner's Dilemma Game."

Computational Economics 35 (3): 211-34. doi:10.1007/s10614-009-9184-4.

Berger, Thomas. 2001. "Agent-Based Spatial Models Applied to Agriculture: A

Simulation Tool for Technology Diffusion, Resource Use Changes and Policy

Analysis." Agricultural Economics, Increasing Efficiency in Production,

Research, Markets and Environmental Management. Selected and edited papers

presented during the XXIV Conference of the International Association of

Agricultural Economists, 25 (2): 245-60. doi:10.1016/S0169-5150(01)00082-2.

Camerer, Colin. 2011. "The Promise and Success of Lab-Field Generalizability in Experimental Economics: A Critical Reply to Levitt and List.” SSRN Scholarly Paper ID 1977749. Rochester, NY: Social Science Research Network. https://papers.ssrn.com/abstract=1977749.

Camerer, Colin, and Teck Hua Ho. 1999. "Experience-Weighted Attraction Learning in Normal Form Games.” Econometrica 67 (4): 827-74. doi:10.1111/14680262.00054.

Cason, Timothy N., Lata Gangadharan, and Charlotte Duke. 2003. "A Laboratory Study of Auctions for Reducing Non-Point Source Pollution." Journal of Environmental Economics and Management 46 (3): 446-71. doi:10.1016/S0095-0696(03)000263.

Cochard, François, Marc Willinger, and Anastasios Xepapadeas. 2005. "Efficiency of Nonpoint Source Pollution Instruments: An Experimental Study." Environmental and Resource Economics 30 (4): 393-422. doi:10.1007/s10640-004-5986-y.

EEA (2007), Europe's Environment-The Fourth Assessment, 2007.

Eisenhauer, Brian.W., Nicholas Stevenson, Christian Weber, and Julia Peterson. "Changing homeowner's lawn care behavior to reduce nutrient losses in New England's urbanizing watersheds Social Science Results Summary." University of New Hampshire Cooperative Extension (2009).

EPA (2007), National Water Quality Inventory: Report to Congress.

Farzin, Y. Hossein, and Jonathan D. Kaplan. 2004. "Nonpoint Source Pollution Control under Incomplete and Costly Information." Environmental and Resource Economics 28 (4): 489-506. doi:10.1023/B:EARE.0000036775.79214.a4.

Fehr, Ernst, and Urs Fischbacher. 2003. "The Nature of Human Altruism.” Nature 425 (6960):785-791.

Feri, Francesco, Bernd Irlenbusch, and Matthias Sutter. 2010. "Efficiency Gains from Team-Based Coordination-Large-Scale Experimental Evidence." The American Economic Review 100 (4):1892-1912.

Fréchette, Guillaume R., and Andrew Schotter. 2015. Handbook of Experimental Economic Methodology. Oxford University Press.

Fischbacher, Urs. 2007. "Z-Tree: Zurich Toolbox for Ready-Made Economic Experiments.” Experimental Economics 10 (2):171-78. https://doi.org/10.1007/s10683-006-9159-4.

Gode, Dhananjay K., and Shyam Sunder. 1993. "Allocative Efficiency of Markets with Zero-Intelligence Traders: Market as a Partial Substitute for Individual 
Rationality." Journal of Political Economy 101 (1): 119-37. doi:10.1086/261868.

Griffin, Ronald C., and Daniel W. Bromley. 1982. "Agricultural Runoff as a Nonpoint

Externality: A Theoretical Development." American Journal of Agricultural Economics 64 (3): 547-52. doi:10.2307/1240648.

Hanley, Nick, Hilary Kirkpatrick, Ian Simpson, and David Oglethorpe. 1998. "Principles for the Provision of Public Goods from Agriculture: Modeling Moorland Conservation in Scotland." Land Economics 74 (1): 102-13. doi: $10.2307 / 3147216$.

Hansen, Lars Gårn. 2002. "Regulation of Non-Point Emissions - A Variance Based Mechanism." Environmental and Resource Economics 21 (4): 303-16. doi:10.1023/A:1015140602916.

Ho, Teck H., Xin Wang, and Colin F. Camerer. 2008. "Individual Differences in EWA Learning with Partial Payoff Information.” The Economic Journal 118 (525): $37-$ 59. doi:10.1111/j.1468-0297.2007.02103.x.

Horan, Richard D., James S. Shortle, and David G. Abler. 1998. “Ambient Taxes When Polluters Have Multiple Choices." Journal of Environmental Economics and Management 36 (2): 186-99. doi:10.1006/jeem.1998.1041.

Isaac, R. Mark, and James M. Walker. 1988. "Group Size Effects in Public Goods Provision: The Voluntary Contributions Mechanism.” The Quarterly Journal of Economics 103 (1):179-99. https://doi.org/10.2307/1882648.

Janssen, Marco A. 2014. "An Agent-Based Model Based on Field Experiments." In Empirical Agent-Based Modelling - Challenges and Solutions, 189-205. Springer, New York, NY. doi:10.1007/978-1-4614-6134-0_10.

Janssen, Marco A., Nicholas P. Radtke, and Allen Lee. 2009. "Pattern-Oriented Modeling of Commons Dilemma Experiments.” Adaptive Behavior 17 (6): 508-23. doi:10.1177/1059712309342488.

Li, Youping. 2013. "The Voluntary-Threat Approach to Control Nonpoint Source Pollution under Uncertainty." Journal of Environmental Management 129 (Supplement C): 69-72. doi:10.1016/j.jenvman.2013.05.047.

Miao, Haoran, Jacob R. Fooks, Todd Guilfoos, Kent D. Messer, Soni M. Pradhanang, Jordan F. Suter, Simona Trandafir, and Emi Uchida. 2016. "The Impact of Information on Behavior under an Ambient-Based Policy for Regulating Nonpoint Source Pollution.” Water Resources Research, April, n/a-n/a. doi:10.1002/2015WR018142.

Miao, Haoran, Jacob, Todd Guilfoos, and Emi Uchida. 2017. "Uncertainty and nonpoint source polluters' behavior under an ambient tax policy." To be submitted.

Schelling, Thomas C. 1971. "Dynamic Models of Segregation." The Journal of Mathematical Sociology 1 (2): 143-86. doi:10.1080/0022250X.1971.9989794.

Segerson, Kathleen. 1988. "Uncertainty and Incentives for Nonpoint Pollution Control." Journal of Environmental Economics and Management 15 (1): 87-98. doi:10.1016/0095-0696(88)90030-7.

Segerson, Kathleen, and JunJie Wu. 2006. "Nonpoint Pollution Control: Inducing FirstBest Outcomes through the Use of Threats." Journal of Environmental Economics and Management 51 (2): 165-84. doi:10.1016/j.jeem.2005.04.007.

Shay, Kathy. 2011. Austin Lawn and Garden Chemical Education Campaign. https://cfpub.epa.gov/npstbx/files/Shay\%202011.pdf 
Shortle, James S., and James W. Dunn. 1986. "The Relative Efficiency of Agricultural Source Water Pollution Control Policies." American Journal of Agricultural Economics 68 (3): 668-77. doi:10.2307/1241551.

Spraggon, John. 2002. "Exogenous Targeting Instruments as a Solution to Group Moral Hazards." Journal of Public Economics 84 (3): 427-56. doi:10.1016/S00472727(01)00088-3.

- 2004. "Testing Ambient Pollution Instruments with Heterogeneous Agents." Journal of Environmental Economics and Management 48 (2): 837-56. doi:10.1016/j.jeem.2003.11.006.

Sun, Bo, Linxiu Zhang, Linzhang Yang, Fusuo Zhang, David Norse, and Zhaoliang Zhu. 2012. "Agricultural Non-Point Source Pollution in China: Causes and Mitigation Measures." Ambio 41 (4): 370-79. doi:10.1007/s13280-012-0249-6.

Suter, Jordan F., Christian A. Vossler, and Gregory L. Poe. 2009. “Ambient-Based Pollution Mechanisms: A Comparison of Homogeneous and Heterogeneous Groups of Emitters.” Ecological Economics, Eco-efficiency: From technical optimisation to reflective sustainability analysis, 68 (6): 1883-92. doi:10.1016/j.ecolecon.2009.01.004.

Suter, Jordan F., Christian A. Vossler, Gregory L. Poe, and Kathleen Segerson. 2008. "Experiments on Damage-Based Ambient Taxes for Nonpoint Source Polluters." American Journal of Agricultural Economics 90 (1): 86-102. doi:10.1111/j.14678276.2007.01055.x.

Taylor, Michael A., Brent Sohngen, Alan Randall, and Helen Pushkarskaya. 2004. "Group Contracts for Voluntary Nonpoint Source Pollution Reductions: Evidence from Experimental Auctions." American Journal of Agricultural Economics 86 (5): 1196-1202.

Vossler, Christian A., Gregory L. Poe, William D. Schulze, and Kathleen Segerson. 2006. "Communication and Incentive Mechanisms Based on Group Performance: An Experimental Study of Nonpoint Pollution Control.” Economic Inquiry 44 (4): 599-613. doi:10.1093/ei/cbj043.

Xepapadeas, A. P. 1992. "Environmental Policy Design and Dynamic Nonpoint-Source Pollution." Journal of Environmental Economics and Management 23 (1): 22-39. doi:10.1016/0095-0696(92)90039-Y.

Xepapadeas, A. P. 1995. "Observability and Choice of Instrument Mix in the Control of Externalities.” Journal of Public Economics 56 (3): 485-98. doi:10.1016/00472727(94)01441-P.

Xepapadeas, Anastasios. 2011. "The Economics of Non-Point-Source Pollution.” Annual Review of Resource Economics 3 (1): 355-73. doi:10.1146/annurev-resource083110-115945. 
Table 3.1: Treatment tables

\begin{tabular}{cccc}
\hline Treatment & Label & Information about others group members' decisions & Uncertainty Level \\
A & $\begin{array}{c}\text { No information, } \\
\text { Certainty } \\
\text { No information, Low } \\
\text { uncertainty } \\
\text { No information, High } \\
\text { certainty }\end{array}$ & No & Certainty \\
C & Information, Certainty & No & Howcertainty \\
$\mathrm{D}$ & $\begin{array}{c}\text { Information, Low } \\
\text { uncertainty } \\
\text { Information, High } \\
\text { certainty }\end{array}$ & Yes & Certainty \\
$\mathrm{F}$ & Yes & Low uncertainty \\
\hline
\end{tabular}


Table 3.2: Function forms and parameterization

\begin{tabular}{|c|c|c|c|c|}
\hline Description & Function Form & $\begin{array}{l}\text { Parameter Values } \\
\text { Certainty }\end{array}$ & $\begin{array}{l}\text { Parameter Values } \\
\text { Low uncertainty }\end{array}$ & $\begin{array}{l}\text { Parameter Values } \\
\text { High uncertainty }\end{array}$ \\
\hline $\begin{array}{l}\text { Profit function } \\
\quad \pi\left(\mathrm{y}_{i}, \mathrm{a}_{i}\right)\end{array}$ & $p^{*} y_{i}-\left(\mathrm{A} y_{i}^{2}+\mathrm{B} a_{i}^{2}\right)+\mathrm{C}$ & $\begin{array}{c}p=6.00, \mathrm{~A}=0.02, \mathrm{~B}= \\
0.05, \mathrm{C}=350\end{array}$ & $\begin{array}{c}p=6, \mathrm{~A}=0.02, \mathrm{~B}= \\
0.05, \mathrm{C}=350\end{array}$ & $\begin{array}{c}p=6, \mathrm{~A}=0.02, \mathrm{~B}=0.05 \\
\mathrm{C}=350\end{array}$ \\
\hline $\begin{array}{l}\text { Emission Function } \\
\qquad \mathrm{r}_{i}\left(\mathrm{y}_{i}, \mathrm{a}_{i}, \varepsilon\right)\end{array}$ & $\left(\mathrm{E} y_{i}^{2}-\mathrm{F} a_{i}^{0.5}\right)^{*} \varepsilon /\left(\mu+\sigma^{2} / 2\right)$ & $\begin{array}{c}\mathrm{E}=1 / 3750, \mathrm{~F}=4 / 75, \varepsilon \\
=\mu+\sigma^{2} / 2\end{array}$ & $\begin{aligned} \mathrm{E}= & 1 / 2750, \mathrm{~F}=2 / 275,, \varepsilon \\
& \sim \log N(1.25,0.5)\end{aligned}$ & $\begin{aligned} \mathrm{E}= & 1 / 2750, \mathrm{~F}=2 / 275, \varepsilon \\
& \sim \log N(0.875,1)\end{aligned}$ \\
\hline $\begin{array}{l}\text { Ambient pollution } \\
\text { function } \mathrm{x}(\mathrm{r})\end{array}$ & $\sum_{i=1}^{\mathrm{I}} r_{i}$ & $\mathrm{I}=6$ & $\mathrm{I}=6$ & $\mathrm{I}=6$ \\
\hline $\begin{array}{l}\text { Damage function } \\
\qquad \mathrm{D}(\mathrm{x})\end{array}$ & $H * x$ & $\mathrm{H}=30$ & $\mathrm{H}=30$ & $\mathrm{H}=30$ \\
\hline $\begin{array}{c}\text { Tax function } \\
\mathrm{T}(\mathrm{x})\end{array}$ & $\mathrm{t} * \mathrm{x}$ & $\mathrm{t}=30$ & $t=30$ & $\mathrm{t}=30$ \\
\hline
\end{tabular}


Table 3.3: Profit-maximizing values of production and abatement

\begin{tabular}{ccccc}
\hline & & & & \\
Management Option & (Expected) Emissions (r) & Abatement (a) & Production $(\mathrm{y})$ & 150.00 \\
Profit $(\pi)$ \\
$\#$ \#6 & 6.00 & 0.00 & 137.81 & 800.00 \\
$\# 4$ & 5.00 & 1.46 & 123.78 & 796.92 \\
$\# 3$ & 4.00 & 2.62 & 107.91 & 785.92 \\
$\# 2$ & 3.00 & 3.93 & 89.32 & 763.81 \\
$\# 1$ & 2.00 & 5.69 & 65.87 & 65.73 \\
\end{tabular}


Table 3.4a: Parameter estimates of the EWA learning model for no-information disclosure treatments

\begin{tabular}{cccc}
\hline Parameters & Treatment A & Treatment B & Treatment C \\
\hline $\boldsymbol{\lambda}$ & $1.72 * *$ & $1.01 * * *$ & $0.22 * * *$ \\
& $(0.87)$ & $(0.16)$ & $(0.08)$ \\
$\boldsymbol{\Phi}$ & $0.88^{* * *}$ & $0.95^{* * *}$ & $0.93 * * *$ \\
& $(0.01)$ & $(0.01)$ & $(0.01)$ \\
$\boldsymbol{\delta}$ & $0.13 * *$ & 0.12 & 0.02 \\
& $(0.07)$ & $(0.07)$ & $(0.13)$ \\
$\mathbf{K}$ & 0.01 & $0.06 * * *$ & $0.38^{* *}$ \\
& $(0.08)$ & $(0.02)$ & $(0.16)$ \\
$\mathbf{N}(\mathbf{0})$ & 4.40 & $1.06 * *$ & $0.30 * *$ \\
& $(3.15)$ & $(0.48)$ & $(0.14)$ \\
\hline
\end{tabular}

$N=1890$ for each treatment. Standard errors in parentheses. $* * * p<0.01, * * p<0.05$, $* p<0.1$

Table 3.4b: Parameter estimates of the EWA learning model for information disclosure treatments

\begin{tabular}{|c|c|c|c|}
\hline Parameters & Treatment D & Treatment E & Treatment $\mathrm{F}$ \\
\hline \multirow[t]{2}{*}{$\lambda$} & $1.45 * * *$ & $1.73 * * *$ & $0.46 * * *$ \\
\hline & $(0.26)$ & $(0.07)$ & $(0.08)$ \\
\hline \multirow[t]{2}{*}{$\Phi$} & $0.90 * * *$ & $0.93 * * *$ & $0.98 * * *$ \\
\hline & 0.11 & $(0.01)$ & $(0.01)$ \\
\hline \multirow[t]{2}{*}{$\boldsymbol{\delta}$} & $0.18 * *$ & 0.00 & 0.12 \\
\hline & $(0.07)$ & $(0.00)$ & $(0.10)$ \\
\hline \multirow[t]{2}{*}{$\kappa$} & 0.03 & 0.00 & $0.12 * * *$ \\
\hline & $(0.03)$ & $(0.00)$ & 0.02 \\
\hline \multirow[t]{2}{*}{$\mathbf{N}(\mathbf{0})$} & $3.06 * * *$ & $4.07 * * *$ & $0.66 * * *$ \\
\hline & $(1.01)$ & 0.71 & $(0.24)$ \\
\hline
\end{tabular}




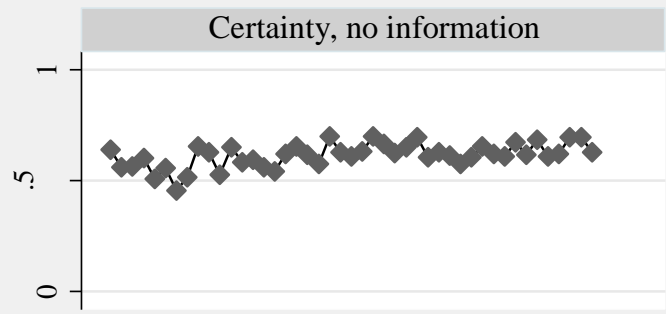

Low uncertainty, no information
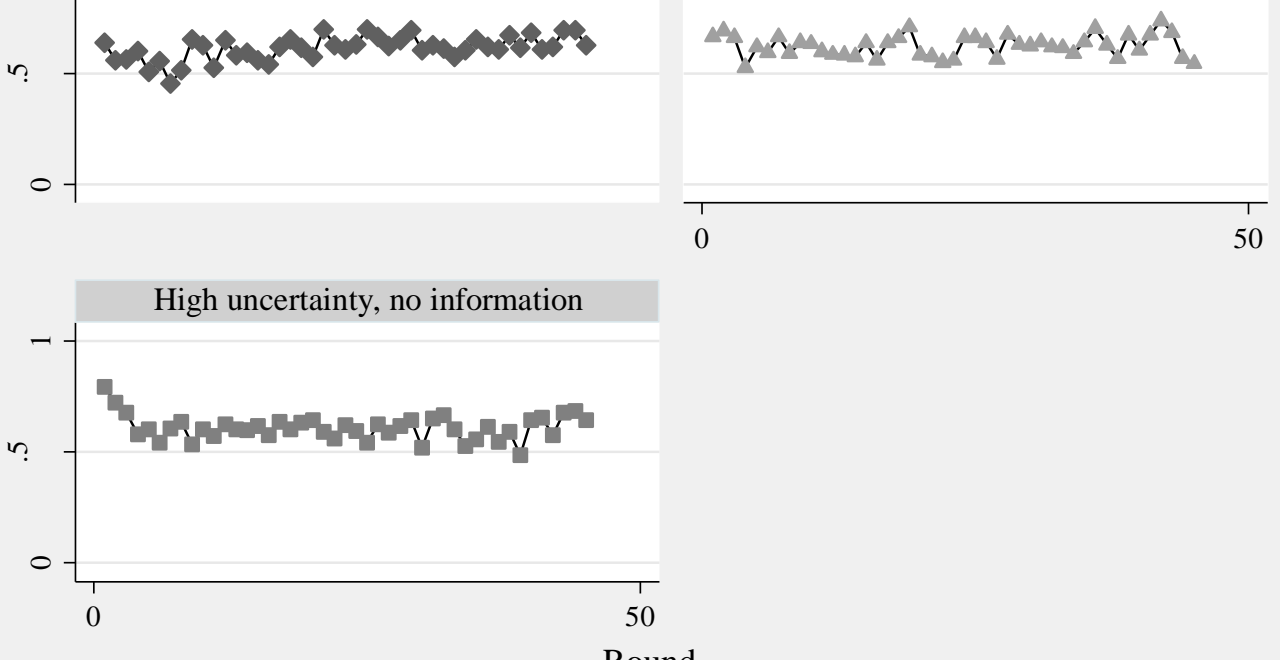

Certainty, no info. Low uncertainty, no info.

High uncertainty, no info.

Figure 3.1a: Average social efficiency over time (No information disclosure)
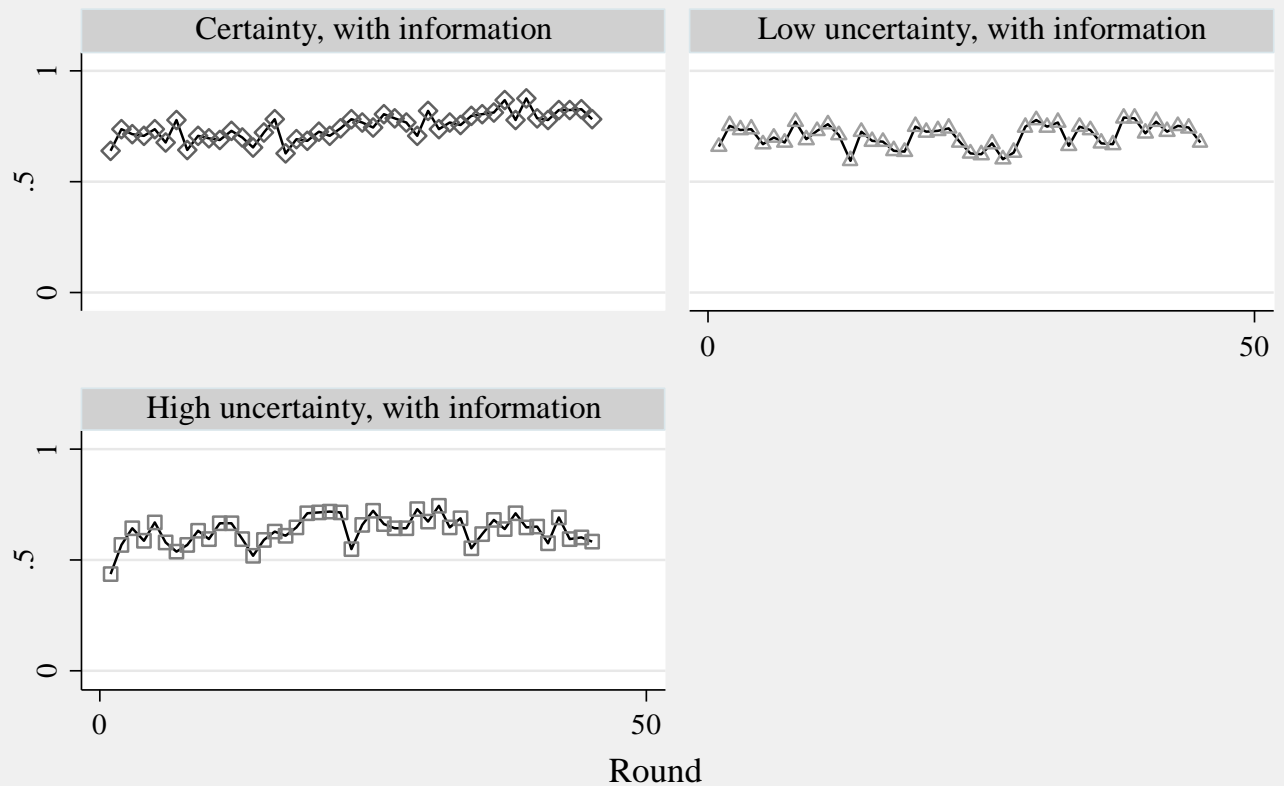

$\diamond-$ Certainty, with info.
- High uncertainty, with info.

Figure 3.1b: Average social efficiency over time (With information disclosure) 

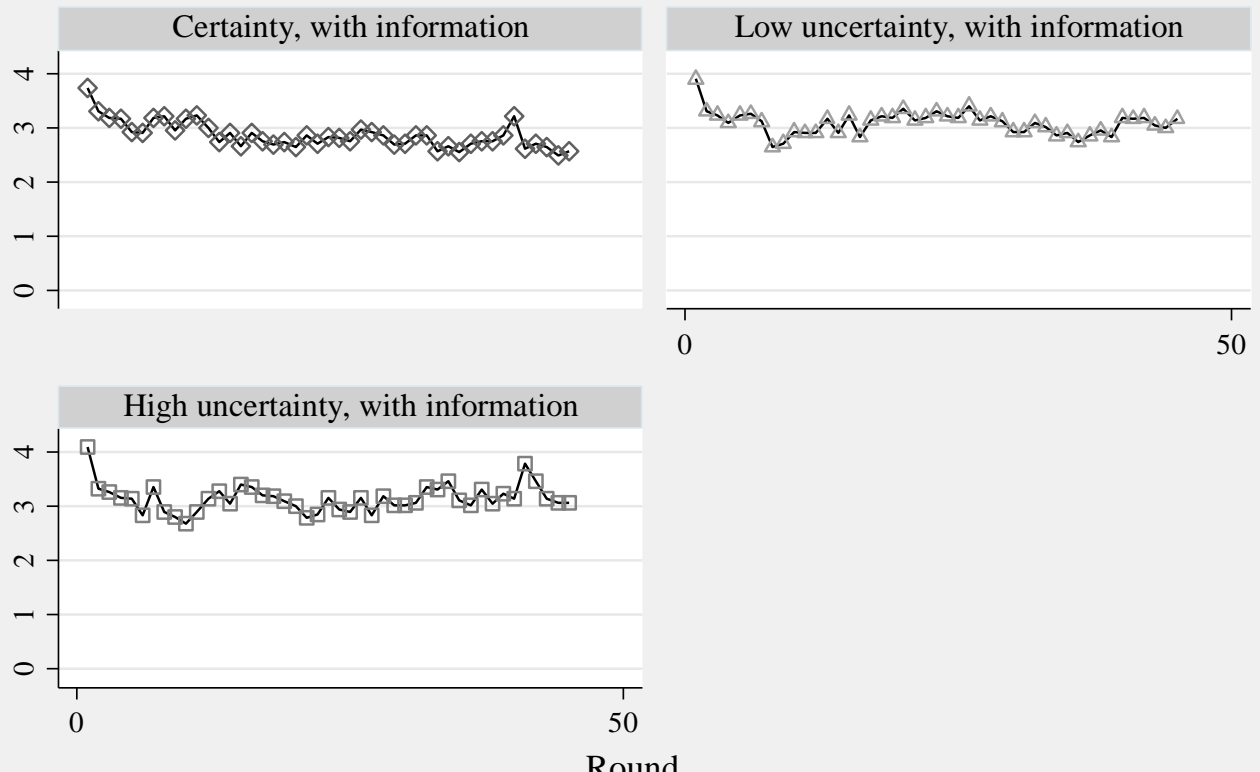

$\diamond-$ Certainty, with info.
$\longrightarrow-$ High uncertainty, with info.

Figure 3.2a: Average individual emission over time (no information disclosure)
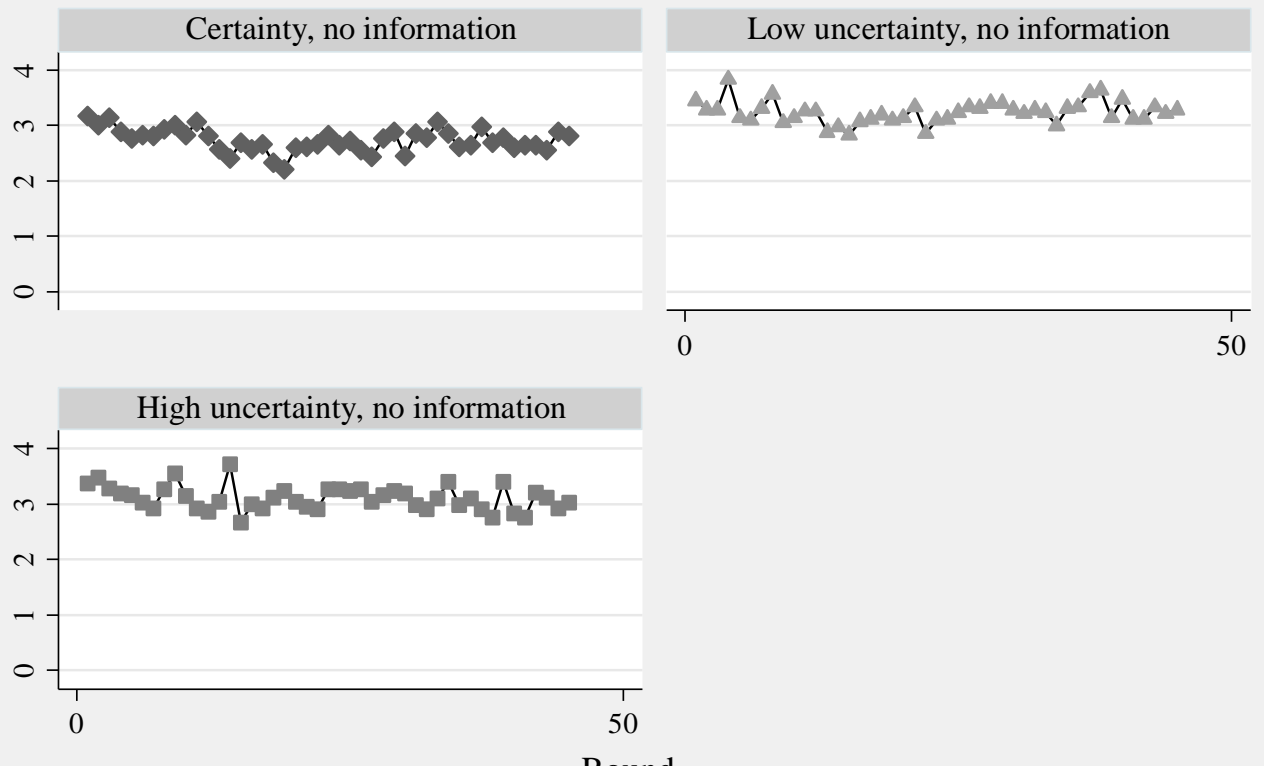

Certainty, no info. Low uncertainty, no info.

High uncertainty, no info.

Figure 3.2b: Average individual emission over time (with information disclosure) 


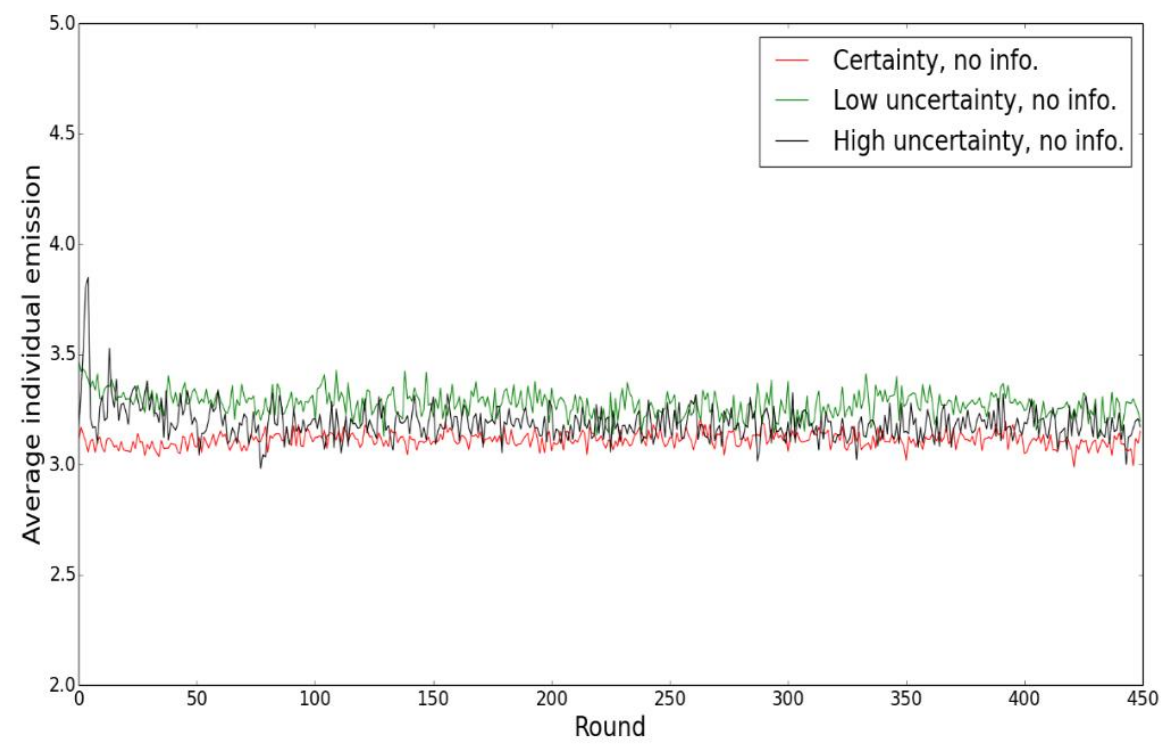

Figure 3.3a: Simulated average individual emission over time (no information disclosure)

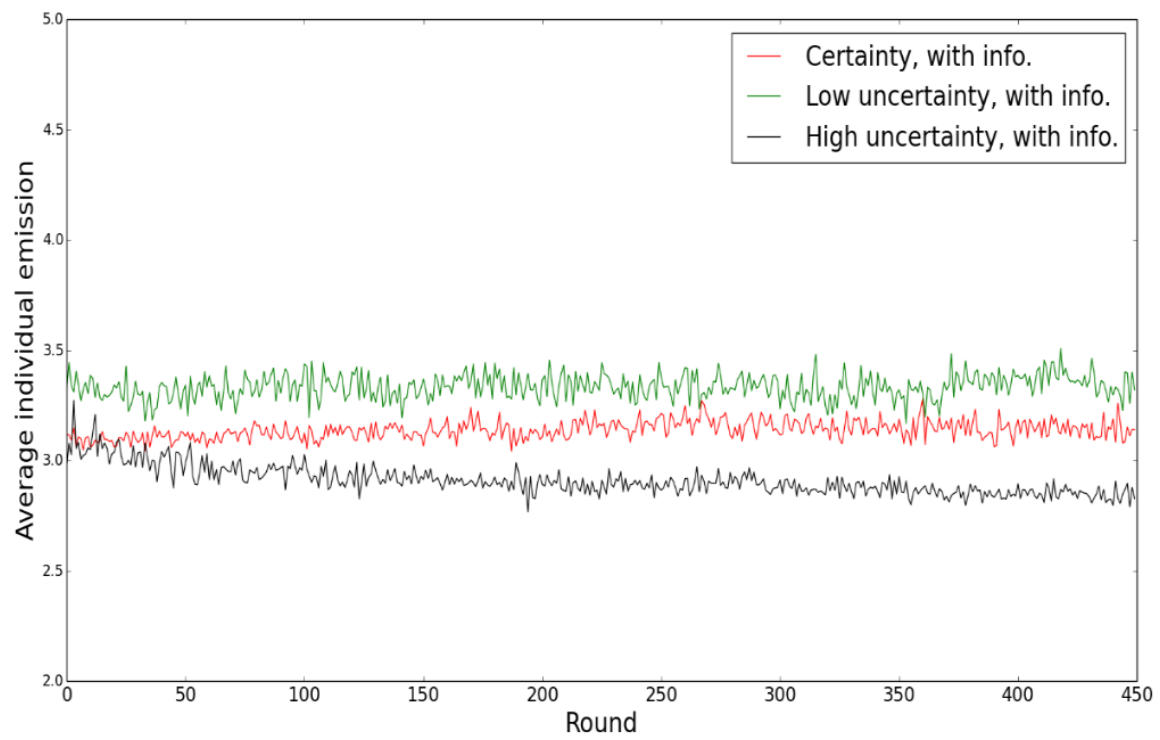

Figure 3.3b: Simulated average individual emission over time (with information disclosure 


\section{POLICY IMPLICATION AND FUTURE DIRECTIONS FOR RESEARCH}

It has been well established that economic inefficiency in the nonpoint source (NPS) pollution problems stems from information asymmetries [Farzin and Kaplan, 2004]. The size of emissions cannot be inferred or observed with reasonable cost because NPS pollution comes from diffuse sources and is in the form of excess fertilizers, herbicides, and insecticides. For example, it is typically prohibitively costly to get information about how much pollution comes from one specific parcel of land in the field, such as a residential lawn or a plot of agricultural land. Information asymmetries between the environmental regulator and those whose activities generate pollution emissions make traditional emission tax or regulation ineffective [Xepapadeas, 2011]. Alternative policies designed by economists to control NPS pollution are generally divided into two categories: regulatory approaches [e.g., ambient-based instruments (Segerson, 1988; Xepapadeas, 1992; Xepapadeas, 1995; Horan et al., 1998; Hansen, 2002) and input taxes (Griffin and Bromley, 1982; Shortle and Dunn, 1986)] and voluntary approaches [e.g., voluntary-threat approach (Segerson and Wu, 2006; Li, 2013), and "pay-the-polluter" approach (Hanley et al., 1998)].

In this dissertation, I examine how information affects NPS polluters' behavior in a voluntary program and under a regulatory policy. In particular, I investigate the effects of informational nudges and financial incentives on homeowners' decisions of choosing lawn care services through a field experiment. Additionally, I test the effects of environmental uncertainty at the individual level and information disclosure on polluters' behavior under an ambient tax policy in a laboratory experiment and one agent-based model. 
In the voluntary program, I find that informational nudges and financial incentives may effectively change homeowners' behavior. However, I find that the two types of intervention are not complementary in promoting environment-friendly behavior. In the laboratory experiment where I use students to represent NPS polluters to test the effectiveness and efficiency of the ambient tax policy, I find that environmental uncertainty at the individual level increases the NPS pollution level and thus jeopardize the efficiency of the ambient tax policy. Also, I discover that information disclosure, giving the laboratory subjects the ability to observe other group members' actions, lower the NPS pollution level. However, information disclosure induces over-abetment behavior, especially when there is an environment with certainty, and therefore lowers the efficiency of the ambient tax policy. When I use agents in the ABM to represent NPS polluters to investigate their behavior in the long run, I discover similar treatment effects of environmental uncertainty. High level of environmental uncertainty, however, creates plenty of negative payoff shocks and induces pollution reduction in the long run when agents can observe group members' behavior.

Results from this dissertation research suggest that information is vital in managing NPS pollution, whether in a voluntary program or under a regulatory policy, but that an environmental regulator should use information carefully and strategically in designing policy to reduce NPS pollution. Current solutions to NPS pollution problem rely heavily on voluntary adoption of best management practices. Financial incentives or technical assistance are often used in the farming sector. Information to nudge environmental friendly behavior to reduce NPS pollution is commonly used in outreach programs. Our results imply a potential tension between informational nudges and 
financial incentives in the context of the lawn care practices. Subsidizing or nudging homeowners may both work to change their behavior so that desired outcomes can be achieved, but both of them can be costly. Using both solutions together do not increase behavior changes significantly in the extensive margin compared to using one of the solutions alone. Thus, our results further imply that the regulator needs to design voluntary programs cautiously to reduce NPS pollution, especially when information nudges and financial incentives are used together. Cost-benefit analysis should be conducted to estimate strengths and weakness of both solutions before any implementation. Also, targeting the right population may help improve the efficiency of policy since results from the voluntary program suggest different strategies work for different people depending on their degree of environmental preferences. Specifically, financial incentives may better change the behavior of people who have already possessed the information of nudges. Conversely, informational nudges may have a significant effect on those people who may develop less environmental awareness.

Recently, budgets used for voluntary programs to reduce NPS pollution has been limited in the United States. Congress has continuously cut the budget tremendously for U.S. Department of Agriculture conservation programs, which are the primary source of funding to support voluntary adoption of best management practices [Shortle et al., 2012]. This increasingly budget-constrained situation underscores the demand for alternative policy approaches if water quality goals are to be achieved. Although according to the literature, there are no applications of ambient-based policy in the field, it is one of the promising policy based on a series of theoretical research and a dozen of empirical analyses in the laboratory. I envision that ambient-based policy 
instruments are the critical solutions to the nonpoint source pollutions because of two advantages. First, they target ambient pollution level and do not require firm-specific information and thus reduce the complication of policy design compared to other proposed policy instruments, such as input-based incentives. Second, they are optimal solutions to both point and nonpoint source pollution so that we do not have to devise two separate incentive systems to reduce pollution if both types of pollution exist in a watershed.

Despite the advantages of ambient-based policy instruments, their efficiency and impact on pollution abatement are sensitive to how those instruments are designed. Based on the results of previous literature and this dissertation, we understand that one subset of ambient-based policy instruments are more effective and efficient than others and several restrictions may help an environmental regulator to improve overall social efficiency. Specifically, the ambient tax seems to be the most suitable one among all the ambient-based policy instruments because of its ability to eliminate the possible inefficient collusive behavior.

Various theoretical and experimental studies to date have allowed to us understand multiple theoretical properties of the ambient tax policy. However, it has yet to be implemented in the field. As the first step, I recommend that the field experiments start with a relatively small watershed with relatively few, homogeneous polluters. Involving too many polluters worsens the balance budgeting between damages and tax payments since the ambient tax is a collective penalty. Also, heterogeneity among polluters decreases social efficiency based previous experimental studies. Moreover, the target which triggers the ambient tax needs to be set with care. Although the theoretical 
literature demonstrates that the target can be arbitrary and some experimental research also suggests that social efficiency does not have significant change when we vary the target, my experimental results provide evidence that a target below the optimum reduces social efficiency most due to over-abatement behavior. Therefore, I conclude that the environmental regulator can choose neither a too low target nor a too high one. If it is too low, it may induce over-abatement behavior because of various reasons, such as communication. If it is too high, the environmental regulator cannot achieve desired environmental outcomes. It is critical to obtain the reliable water quality data to approximate the social optimum and may need to be flexible so that the target can be adjusted based on long-term trends in water quality patterns, specific cases, and policy outcomes. Also, previous literature suggests that it is crucial to reduce error and increase measurement accuracy when monitoring ambient pollution level because uncertainty in ambient pollution measurement lowers social efficiency. Furthermore, my experimental results indicate that environmental uncertainty may induce nonpoint source polluters' under-abatement behavior, and therefore, this vital feature of NPS pollution also requires the flexible design of the target.

Future research on ambient-based policy instruments may focus on searching for close analogs to such instruments. Ideally, different policy designs need to be tested using field experiments. If close analogs cannot be found in the field, demonstration projects that further show their effectiveness and efficiency may be helpful for decision making. While all these future research directions are temporarily infeasible, we may step back, look into the existing data and find better models to describe subjects' behavior under the ambient-based policy. We can further test the policy using the ABM 
techniques, which may break through the limitations of laboratory experiments and expand the possibility of research such as integrating behavior in the laboratory with real-world environmental data to produce case-specific results. 


\section{References}

Farzin, Y. Hossein, and Jonathan D. Kaplan. 2004. "Nonpoint Source Pollution Control under Incomplete and Costly Information." Environmental and Resource Economics 28 (4):489-506. https://doi.org/10.1023/B:EARE.0000036775.79214.a4.

Griffin, Ronald C., and Daniel W. Bromley. 1982. "Agricultural Runoff as a Nonpoint Externality: A Theoretical Development." American Journal of Agricultural Economics 64 (3):547-52. https://doi.org/10.2307/1240648.

Hanley, Nick, Hilary Kirkpatrick, Ian Simpson, and David Oglethorpe. 1998. "Principles for the Provision of Public Goods from Agriculture: Modeling Moorland Conservation in Scotland." Land Economics 74 (1):102-13. https://doi.org/10.2307/3147216.

Hansen, Lars Gårn. 2002. "Regulation of Non-Point Emissions - A Variance Based Mechanism." Environmental and Resource Economics 21 (4):303-16. https://doi.org/10.1023/A:1015140602916.

Horan, Richard D., James S. Shortle, and David G. Abler. 1998. “Ambient Taxes When Polluters Have Multiple Choices." Journal of Environmental Economics and Management 36 (2):186-99. https://doi.org/10.1006/jeem.1998.1041.

Li, Youping. 2013. "The Voluntary-Threat Approach to Control Nonpoint Source Pollution under Uncertainty.” Journal of Environmental Management 129 (Supplement C):69-72. https://doi.org/10.1016/j.jenvman.2013.05.047.

Segerson, Kathleen. 1988. "Uncertainty and Incentives for Nonpoint Pollution Control." Journal of Environmental Economics and Management 15 (1):8798. https://doi.org/10.1016/0095-0696(88)90030-7.

Segerson, Kathleen, and JunJie Wu. 2006. "Nonpoint Pollution Control: Inducing First-Best Outcomes through the Use of Threats." Journal of Environmental Economics and Management 51 (2):165-84. https://doi.org/10.1016/j.jeem.2005.04.007.

Shortle, James S., and James W. Dunn. 1986. "The Relative Efficiency of Agricultural Source Water Pollution Control Policies.” American Journal of Agricultural Economics 68 (3):668-77. https://doi.org/10.2307/1241551.

Shortle, James S., Marc Ribaudo, Richard D. Horan, and David Blandford. 2012. "Reforming Agricultural Nonpoint Pollution Policy in an Increasingly BudgetConstrained Environment." Environmental Science \& Technology 46 (3):1316-25. https://doi.org/10.1021/es2020499.

Xepapadeas, A. P. 1992. "Environmental Policy Design and Dynamic NonpointSource Pollution." Journal of Environmental Economics and Management 23 (1):22-39. https://doi.org/10.1016/0095-0696(92)90039-Y.

Xepapadeas, A. P. 1995. "Observability and Choice of Instrument Mix in the Control of Externalities." Journal of Public Economics 56 (3):485-98. https://doi.org/10.1016/0047-2727(94)01441-P.

Xepapadeas, Anastasios. 2011. "The Economics of Non-Point-Source Pollution." Annual Review of Resource Economics 3 (1):355-73. https://doi.org/10.1146/annurev-resource-083110-115945. 


\section{APPENDICES}

\section{Appendix I: Field experiment survey}

North East Water Resources Network Field Experiment Survey

[Italic part in this appendix was not shown in the online survey.]

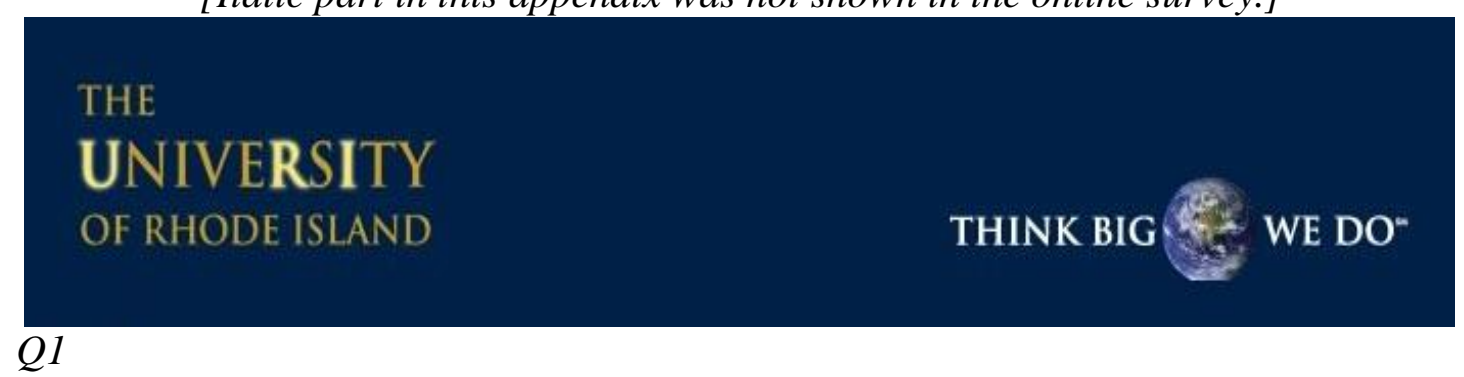

Welcome to the University of Rhode Island's survey on lawn care and sustainable practices.

Thank you for your participation in this important survey. Your response will help us understand how homeowners make decisions about lawn care practices. Before we begin, please answer a few eligibility questions.

Please click 'Next page $>>$ ' to begin. 
Screening questions: $(Q 2-Q 7)$

Q2

Which state do you live in? (Please choose one)

O Rhode Island (1)

O Massachusetts (2)

O Connecticut (3)

O Other (4) 


\section{Display This Question:}

If Which state do you live in? Massachusetts Is Selected

Q3

What is your zip code? (Please choose one)

A list of zip codes was displayed.

Display This Question:

If Which state do you live in? Connecticut Is Selected

Q4

What is your zip code? (Please choose one)

A list of zip codes was displayed. 


\section{Display This Question:}

If Which state do you live in? Other Is Not Selected And What is your zip code? - Other Is Not Selected And What is your zip code? - Other Is Not Selected Q5

Does your current residence have a lawn?

O Yes (1)

O No (2) 


\section{Display This Question:}

If Which state do you live in? Other Is Not Selected

And What is your zip code? - Other Is Not Selected

And Does your current residence have a lawn? No Is Not Selected

And What is your zip code? - Other Is Not Selected

Q6

Who primarily maintains your lawn? (Please choose one)

O Myself/Other member(s) of my household (1)

O Lawn care company (2)

O Landlord/Complex management (3)

O Friend/Neighbor (4)

O Someone else but not a professional (5)

Other (Please specify) (6) 


\section{Display This Question:}

If Who maintains your lawn? Lawn care company Is Selected $Q 7$

Which lawn care company did you hire in 2016? (Please choose one)

O A Cut Above (1)

O A. Paliotta (2)

O Countyview Landscaping (3)

O Cryan Landscaping (4)

O Dana Designs (5)

O Dapontes Landscaping (6)

O It's About Thyme Landscaping (7)

O McGeoghs Turf Mgt (8)

O Murdock Landscaping (9)

O SeaScape LawnCare (10)

O None the above (11) 
Introduction and Survey Part I (Q8 - Q17)

\section{THE}

\section{UNIVERSITY}

OF RHODE ISLAND

\section{THINK BIG

\section{Q8}

Dear Homeowner,

Welcome to the University of Rhode Island's survey on residential lawn care. This survey is part of a study on decisions related to water quality funded by the National Science Foundation. The results of this survey will help us understand how homeowners make decisions about lawn care practices in southern New England.

This survey will take about 15 minutes to complete. As a small thank you gift, all participants who are eligible for the study will receive a \$2 Amazon e-gift card. The gift card will be emailed to you. You can then use it yourself or donate to a school/organization of your choice.

Please be assured that the information you provide is completely confidential. It will not be disclosed to anyone without your permission. Study findings will only be presented in summaries where individual answers cannot be identified.

Your participation is very important to us if we are to gain a fair and accurate understanding of households' decisions and opinions. However, you can choose not to answer any questions or withdraw from the study at any time.

If you have any questions, please feel free to contact Dr. Emi Uchida (Associate Professor, Environmental and Natural Resource Economics, University of Rhode Island). You may reach her by email (euchida@uri.edu) or by phone (401-874-4586). In addition, you may contact the office of the Vice President for Research and Economic Development, 70 Lower College Rd., Suite 2, Kingston, RI (401-874-4328). This survey has been reviewed according to University of Rhode Island Institutional Review Board procedures for research involving human subjects.

By clicking the following "Next page $>>$ " button, you agree that:

- You have read the above information;

- You understand that your participation is voluntary;

- You are at least 18 years of age. 
$Q 9$

Part I: Your lawn care practices in the year 2016

In this section, we will ask a few questions about your lawn care practices in the year 2016. 


\section{Display This Question:}

If Who maintains your lawn? Lawn care company Is Selected

Q10 You informed us that you hired a lawn care company to maintain your lawn in 2016. Please tell us which of the following services were provided by the lawn care company. (Choose all that apply)

$\square$ Watering (1)

Fertilizing (2)

Mowing (3)

$\square$ Aeration (4)

$\square$ Seeding (5)

Insect control (6)

Disease control (7)

Weed control (8)

- Crabgrass control (9)

Other (Please specify) (10) 


\section{Display This Question:}

If Who maintains your lawn? Lawn care company Is Selected Q11

Which of the following actions were used by the lawn care company you hired in 2016 ?

\begin{tabular}{|c|c|c|c|}
\hline & Yes (1) & No (2) & I don't know (3) \\
\hline Conducted a soil test & $\mathrm{O}$ & $\mathrm{O}$ & $\mathrm{O}$ \\
\hline $\begin{array}{l}\text { Used conventional } \\
\text { fertilizers }\end{array}$ & 0 & O & $\mathrm{O}$ \\
\hline $\begin{array}{l}\text { Used slow release } \\
\text { nitrogen fertilizer }\end{array}$ & 0 & 0 & $\mathrm{O}$ \\
\hline $\begin{array}{ll}\text { Used } & \text { organic } \\
\text { fertilizers } & \end{array}$ & 0 & 0 & 0 \\
\hline $\begin{array}{l}\text { Left your grass } \\
\text { clippings on the } \\
\text { lawn }\end{array}$ & 0 & 0 & O \\
\hline $\begin{array}{l}\text { Mowed your lawn } \\
\text { between } 3 \text { to } 4 \\
\text { inches }\end{array}$ & 0 & 0 & 0 \\
\hline $\begin{array}{lll}\text { Hand-watered } & \text { spots } \\
\text { during } & \text { very } & \text { dry } \\
\text { periods } & & \end{array}$ & 0 & 0 & 0 \\
\hline $\begin{array}{l}\text { Cleaned driveway } \\
\text { and sidewalks after } \\
\text { applying fertilizer }\end{array}$ & 0 & 0 & 0 \\
\hline $\begin{array}{l}\text { Did not treat under } \\
\text { windy or rainy } \\
\text { conditions }\end{array}$ & 0 & 0 & $\mathrm{O}$ \\
\hline $\begin{array}{l}\text { Spot treated weeds } \\
\text { with herbicide }\end{array}$ & O & $\mathrm{O}$ & $\mathrm{O}$ \\
\hline
\end{tabular}




\section{Display This Question:}

If Who maintains your lawn? (Please choose one) Myself/Other member(s) of my household Is Selected

Or Who maintains your lawn? (Please choose one) Friend/Neighbor Is Selected

Or Who maintains your lawn? (Please choose one) Someone else but not a professional Is Selected

Or Who maintains your lawn?(Please choose one) Other (Please specify) Is Selected

Q12

Which of the following actions did your household or the person managing your lawn take to maintain your lawn in 2016? (Choose all that apply)

Watering (1)

Fertilizing (2)

Mowing (3)

Aerating (4)

- Seeding (5)

口 Insect control (6)

Disease Control (7)

W Weed control (8)

C Crabgrass control (9)

Other (Please specify) (10) 


\section{Display This Question:}

If Who maintains your lawn? Myself/Other member(s) of my household Is Selected...

Q13

Which of the following practices did your household or the person managing your lawn follow to maintain your lawn in 2016 ?

\begin{tabular}{|c|c|c|c|}
\hline & Yes (1) & No (2) & I don't know (3) \\
\hline Conducted a soil test & $\mathrm{O}$ & $\mathrm{O}$ & $\mathrm{O}$ \\
\hline $\begin{array}{l}\text { Used conventional } \\
\text { fertilizers }\end{array}$ & $\mathrm{O}$ & $\mathrm{O}$ & $\mathrm{O}$ \\
\hline $\begin{array}{l}\text { Used slow release } \\
\text { nitrogen fertilizer }\end{array}$ & $\mathrm{O}$ & $\mathrm{O}$ & $\mathrm{O}$ \\
\hline $\begin{array}{l}\text { Used organic } \\
\text { fertilizers }\end{array}$ & $\mathrm{O}$ & $\mathrm{O}$ & O \\
\hline $\begin{array}{l}\text { Left your grass } \\
\text { clippings on the } \\
\text { lawn }\end{array}$ & $\mathrm{O}$ & $\mathrm{O}$ & $\mathrm{O}$ \\
\hline $\begin{array}{l}\text { Mowed your lawn } \\
\text { between } 3 \text { to } 4 \\
\text { inches }\end{array}$ & $\mathrm{O}$ & $\mathrm{O}$ & $\mathrm{O}$ \\
\hline $\begin{array}{l}\text { Hand-watered spots } \\
\text { during very dry } \\
\text { periods }\end{array}$ & $\mathrm{O}$ & $\mathrm{O}$ & $\mathrm{O}$ \\
\hline $\begin{array}{l}\text { Cleaned driveway } \\
\text { and sidewalks after } \\
\text { applying fertilizer }\end{array}$ & $\mathrm{O}$ & $\mathrm{O}$ & $\mathrm{O}$ \\
\hline $\begin{array}{l}\text { Did not treat under } \\
\text { windy or rainy } \\
\text { conditions }\end{array}$ & $\mathrm{O}$ & $\mathrm{O}$ & $\mathrm{O}$ \\
\hline $\begin{array}{l}\text { Spot treated weeds } \\
\text { with herbicide }\end{array}$ & $\mathrm{O}$ & $\mathrm{O}$ & $\mathrm{O}$ \\
\hline
\end{tabular}

\section{Display This Question:}

\section{If Who maintains your lawn? Lawn care company Is Selected}

Q14

How much did your household spend to hire the lawn care company in 2016? (In US dollars) (Please enter a NUMBER in the box.) 


\section{Display This Question:}

If Who maintains your lawn? (Please choose one) Myself/Other member(s) of my household Is Selected

Or Who maintains your lawn? (Please choose one) Friend/Neighbor Is Selected

Or Who maintains your lawn? (Please choose one) Someone else but not a professional Is Selected

Or Who maintains your lawn? (Please choose one) Other (Please specify) Is Selected

Q15 How much did your household or the person managing your lawn spend in TOTAL in 2016 for your lawn care? (In US Dollars). Please include expenses for fertilizers, pesticides, seeds and other materials you can think of. (Do not include machinery.) (Please enter a NUMBER in the box.) 


\section{Display This Question:}

If Who maintains your lawn? (Please choose one) Myself/Other member(s) of my household Is Selected

Or Who maintains your lawn? (Please choose one) Someone else but not a professional Is Selected

Or Who maintains your lawn? (Please choose one) Friend/Neighbor Is Selected

Or Who maintains your lawn? (Please choose one) Other (Please specify) Is Selected

Q16 How many hours per month on average did your household or the person managing your lawn spend on maintaining your lawn last season? (Please enter a NUMBER in the box.) 
Q17

What is the size of your lawn in ACRES? Please provide your best estimate. (Please enter a NUMBER in the box. For example, if your lawn is half an acre, please enter 0.5.)

For your reference:

- One football field (including the two end zones) $=1.3$ acres

- An official basketball court $=0.1$ acre .

- Average floor area in new single-family houses in the northeast U.S. in $2010=0.06$ acre. 
Survey Part II: treatments (Information treatments + Financial Incentives)

$Q 18$

\section{Part II: About Green Certified Lawn Care Companies}

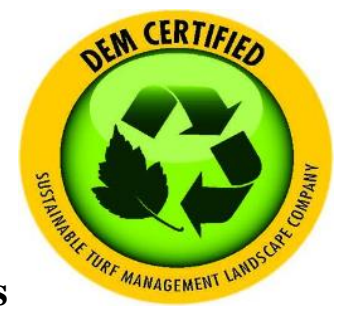

With proper care, it is possible to have a sustainable, responsible and healthy lawn.

In this section, you will find important information about services provided by 'greencertified' lawn care companies. Please read the information carefully. We will ask a few questions following the information. 
One of the following three treatments will be randomly displayed at the same probability for each survey.

Information Treatment $1(Q 19-Q 28)$

Q19

Since 2015, Rhode Island (RI) has a "Green Certification" program for lawn care companies. As of January 2017, 10 lawn care companies are green-certified by the RI Department of Environmental Management.

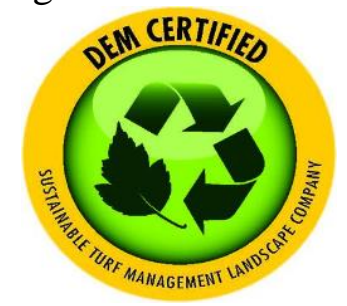

To be certified, lawn care companies must fulfill selected best management practices to protect water quality, conserve water, and contribute to the sustainability of the landscape.

Here are some practices the green-certified companies have adopted:

- mow appropriately (maintain a lawn height of 3-4 inches),

- fertilize appropriately (e.g., fertilize based on a soil test, use non-phosphorous, slow-release fertilizer),

- use safer pesticides (e.g., use mostly granular pesticides to reduce product drift and customer exposure) etc. when maintaining lawns.

Green-certified lawn care companies need to adopt additional best management practices to get re-certified every two years.

The NEXT graphic summarizes these practices. 
Here is a summary of what GREEN-CERTIFIED lawn care companies do.

CERTIFIED LAWN CARE COMPANIES MUST FULFILL BEST MANAGEMENT PRACTICES TO PROTECT WATER QUALITY:

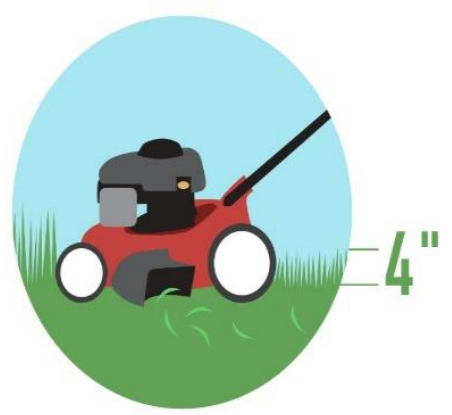

MOW PROPERLY

Maintain a lawn height of 3 to 4 inches.

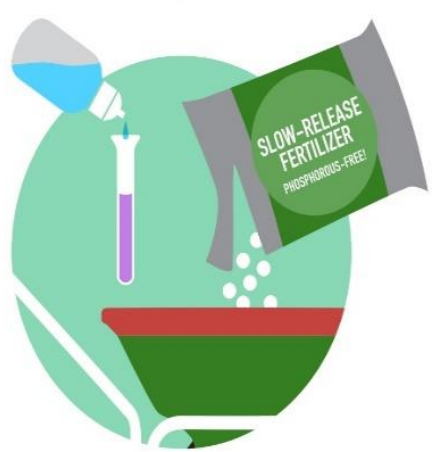

FERTILIZE PROPERLY

Fertilize based on a soil test, and use slow-release, phosphorous-free fertilizer.

Remove fertilizer from driveways, walkways, and roads after application.

Leave buffers untreated around water bodies and wetlands.

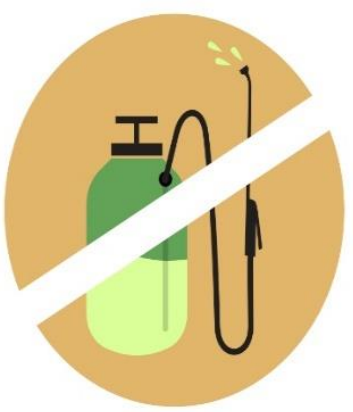

SAFER PESTICIDES

Use granular pesticides to reduce airborne particles and human exposure.

Granular pesticides pose less risk of runoff or leaching. 


\section{Q21}

If you would like to know more about the lawn care practices commonly adopted by green-certified lawn care, here are a few reputable sources*:

University of Rhode Island's Stormwater Solutions:

web.uri.edu/riss/take-action/simple-steps-at-home/around-the-yard/\#FertilizeSparingly University of Connecticut's Sustainable Landscaping:

http://www.sustainability.uconn.edu/sustain/turf/intro.html

University of Massachusetts' Turf Program:

https://ag.umass.edu/turf/publications-resources/best-management-practices

If you decide to check these websites out now, please click the above links and come back to the survey within a few minutes to complete.

*Please note that the best management practices on the websites above are examples of suggested practices. Each certified company chooses a set of practices to get greencertified and may not all adopt the same practice.

Did you check one or more of those websites?

O Yes, I did. (1)

O No, I did not. (2) 
In your opinion, which of the following lawn care practices do you think is effective in sustaining water quality? (Choose all that apply)

$\square$ Conduct a soil test (1)

Maintain a lawn height of 3-4 inches (2)

Fertilize properly using non-phosphorous, slow-release fertilizer (3)

Clean up fertilizer from driveways, walkways, and roads after application (4)

Use safer pesticides and do not spread more than needed (5)

$\square$ Leave areas untreated around water bodies and wetlands (6)

$\square$ None the above (7) 
Q23

Were you aware that Rhode Island has a Green Certification program for lawn care companies?

O Yes (1)

O No (2)

\begin{tabular}{r|rrr} 
Q23 & Freq. & Percent & Cum. \\
\hline 1 & 125 & 23.19 & 23.19 \\
2 & 414 & 76.81 & 100.00 \\
\hline Total & 539 & 100.00 &
\end{tabular}

\section{Display This Question:}

If Who primarily maintains your lawn? (Please choose one) Lawn care company Is Selected

And How much did your household spend to hire the lawn care company in 2016? (In US dollars)\&nbsp; \&nbsp;(Please enter a NUMBER in the box.) \$ Is Not Empty Q24

There are several green-certified lawn care companies that serve your area. If you were to hire a green certified lawn care company to manage your lawn in 2017, how much would you be willing to pay for their services for the entire season?

You stated that you paid $\$ x$ for your services in 2016 . Please use your payment for lawn care in 2016 as a reference.

Imagine that the green-certified lawn care companies will offer you the same services as the company you hired in 2016, but use best management practices that minimize the impact on water quality. Examples include:

- maintain a lawn height of 3-4 inches

- water efficiently

- fertilize properly using non-phosphorous, slow-release fertilizer

- use safer pesticides and do not spread more than needed. 


\section{Display This Question:}

If Who primarily maintains your lawn? (Please choose one) Lawn care company Is Not Selected

And Who primarily maintains your lawn? (Please choose one) Landlord/Complex management Is Not Selected

And How much did your household or the person managing your law spend in TOTAL in 2016 for your lawn care? (In US Dollars) Is Not Empty

And How many hours per month on average did your household or the person managing your ... Is Not Empty

Q25

There are several green-certified lawn care companies that serve your area. If you were to hire a green certified lawn care company to manage your lawn in 2017, how much would you be willing to pay for these services for the entire season?

Imagine that a green-certified company will offer you the following eco-friendly services:

- Five applications of fertilizer from early spring to late fall, at a proper rate, to avoid over fertilizing

- Use organic, slow-release fertilizer with micronutrients

- Broad-leaf weed and crabgrass control

- Pelletized limestone (early fall)

- Removal of materials from walks \& drives to minimize runoff.

For your information, you stated that your household spent $\$ \mathrm{x}$ annually and $\mathrm{x}$ hour(s) each month on average to take care of your lawn in 2016. Please use these figures as a reference. 


\section{Display This Question:}

If Who primarily maintains your lawn? (Please choose one) Lawn care company Is Selected

And How much did your household spend to hire the lawn care company in 2016? (In US dollars) \&nbsp; \&nbsp;(Please enter a NUMBER in the box.) \$ Is Empty Q26

There are several green-certified lawn care companies that serve your area. If you were to hire a green certified lawn care company to manage your lawn in 2017, how much would you be willing to pay for their services for the entire season?

Imagine that the green-certified lawn care companies will offer you the same services as the company you hired in 2016, but use best management practices that minimize the impact on water quality. Examples include:

- maintain a lawn height of 3-4 inches

-water efficiently

- fertilize properly using non-phosphorous, slow-release fertilizer

- use safer pesticides and do not spread more than needed. 


\section{Display This Question:}

If Who primarily maintains your lawn? (Please choose one) Lawn care company Is Not Selected

And Who primarily maintains your lawn? (Please choose one) Landlord/Complex management Is Not Selected

And How much did your household or the person managing your lawn spend in TOTAL in 2016 for your lawn care... \$ Is Empty

Q27

There are several green-certified lawn care companies that serve your area. If you were to hire a green certified lawn care company to manage your lawn in 2017, how much would you be willing to pay for these services for the entire season?

Imagine that a green-certified company will offer you the following eco-friendly services: - Five applications of fertilizer from early spring to late fall, at a proper rate to avoid over fertilizing

- Use organic, slow-release fertilizer with micronutrients

- Broad-leaf weed and crabgrass control

- Pelletized limestone (early fall)

- Removal of materials from walks \& drives to minimize runoff. 


\section{Display This Question:}

If Who primarily maintains your lawn? (Please choose one) Lawn care company Is Not Selected

And Who primarily maintains your lawn? (Please choose one) Landlord/Complex management Is Not Selected

And There are several green-certified lawn care companies that serve your area. If you were to hire...Is Not Displayed

And How many hours per month o... Is Empty

Q28

There are several green-certified lawn care companies that serve your area. If you were to hire a green certified lawn care company to manage your lawn in 2017, how much would you be willing to pay for these services for the entire season?

Imagine that a green-certified company will offer you the following eco-friendly services:

- Five applications of fertilizer from early spring to late fall, at a proper rate, to avoid over fertilizing

- Use organic, slow-release fertilizer with micronutrients

- Broad-leaf weed and crabgrass control

- Pelletized limestone (early fall)

- Removal of materials from walks \& drives to minimize runoff. 
Information Treatment $2(Q 29-Q 42)$

$Q 29$

\section{Part II---Section 1: Your lawn care and water quality}

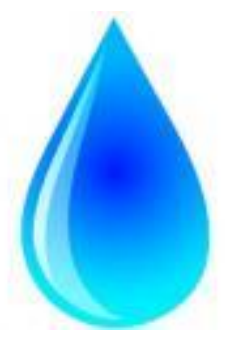

Here you will find information about how your lawn care can affect local water quality depending on how you manage it. Please read the information carefully. We will ask you one question following the information. 
$Q 30$

\section{WHAT DOES YOUR O 74\% Ferpillize} FERTILLZER DO?

A green lawn may look nice, but too much fertilizer can have unintended consequences.

Proper lawn care is crucial in avoiding this!

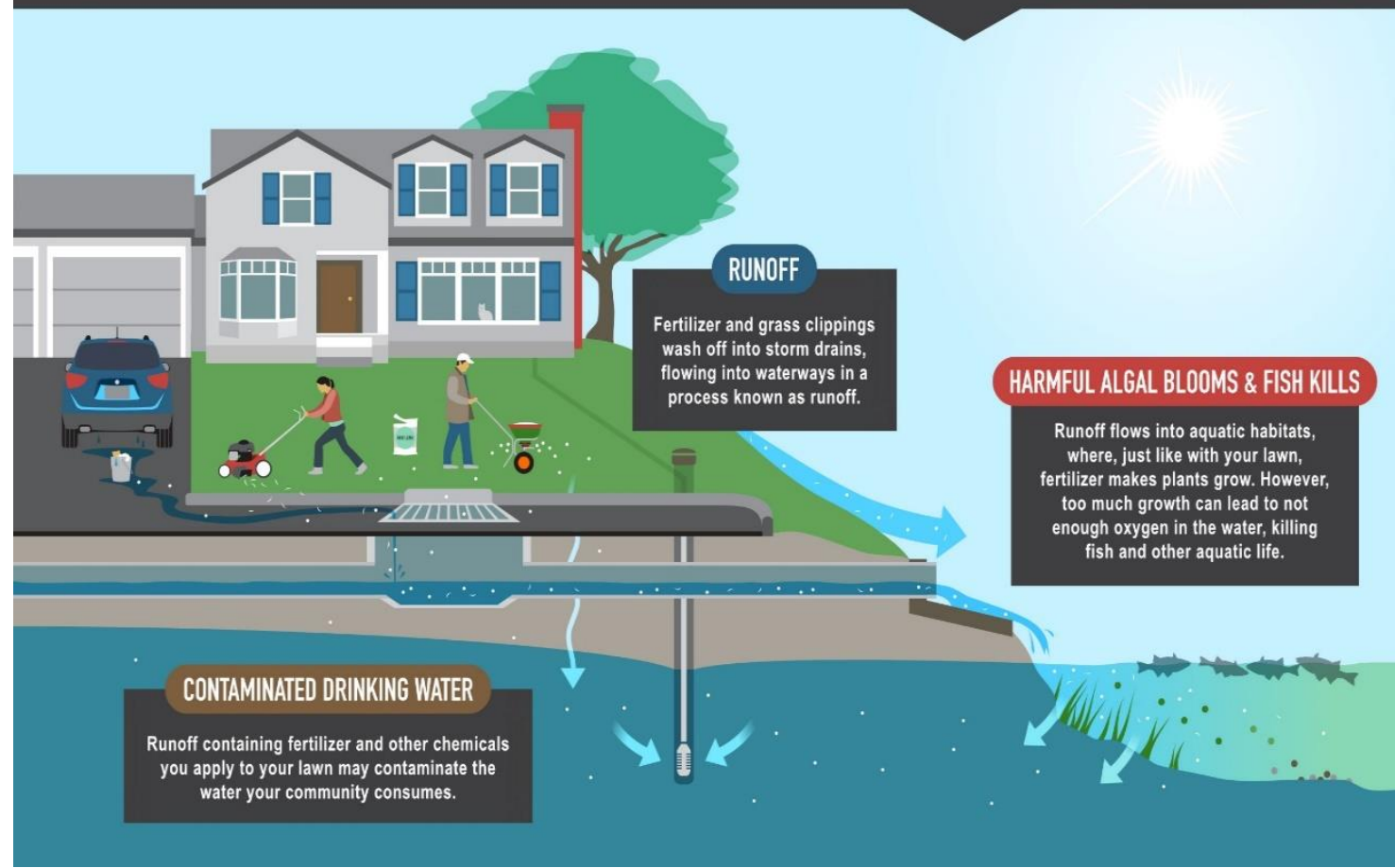

Data Sources:

https://cfpub.epa.gov/npstbx/files/Norming_Survey_LawnCare_NewEngland_July201 $\underline{0 . p d f}$

http://www.stormwatercenter.net/Practice/131-Homeowner\%20Survey.pdf 
Q31

In your opinion, which message from the previous figure was the most surprising to you? (Choose one)

Improper fertilizer can be harmful to our drinking water, fish and other aquatic life. (1)

Proper lawn care can make a difference in protecting our drinking water, fish and other aquatic life. (2)

74\% of households fertilize their lawns. (3)

Only $2 \%$ of households conduct a soil test before fertilizing their lawns. (4)

$40 \%$ of the households use the whole bag of fertilizer to avoid storage. (5)

None of the above were surprising to me. (6) 
Q32

Part II---Section 2: About Green Certified Lawn Care Companies

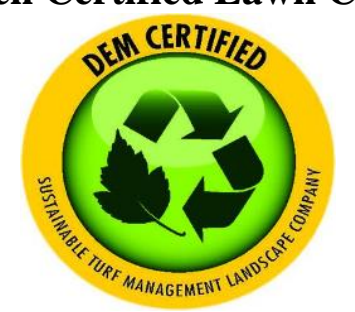

With proper care, it is possible to have a sustainable, responsible and healthy lawn.

In this section, you will find important information about services provided by 'greencertified' lawn care companies. Please read the information carefully. We will ask a few questions following the information. 
Q33

Since 2015, Rhode Island (RI) has a "Green Certification" program for lawn care companies. As of January 2017, 10 lawn care companies are green-certified by the RI Department of Environmental Management.

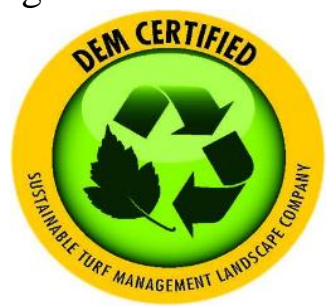

To be certified, lawn care companies must fulfill selected best management practices to protect water quality, conserve water, and contribute to the sustainability of the landscape.

Here are some practices the green-certified companies have adopted:

- mow appropriately (maintain a lawn height of 3-4 inches),

- fertilize appropriately (e.g., fertilize based on a soil test, use non-phosphorous, slow-release fertilizer),

- use safer pesticides (e.g., use mostly granular pesticides to reduce product drift and customer exposure) etc. when maintaining lawns.

Green-certified lawn care companies need to adopt additional best management practices to get re-certified every two years.

The NEXT graphic summarizes these practices. 
Q34

Here is a summary of what GREEN-CERTIFIED lawn care companies do.

CERTIFIED LAWN CARE COMPANIES MUST FULFILL BEST MANAGEMENT PRACTICES TO PROTECT WATER QUALITY:

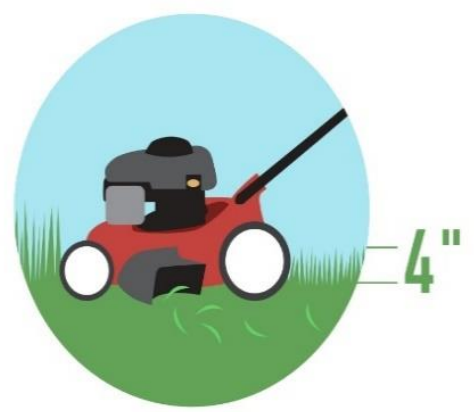

MOW PROPERLY

Maintain a lawn height of 3 to 4 inches.

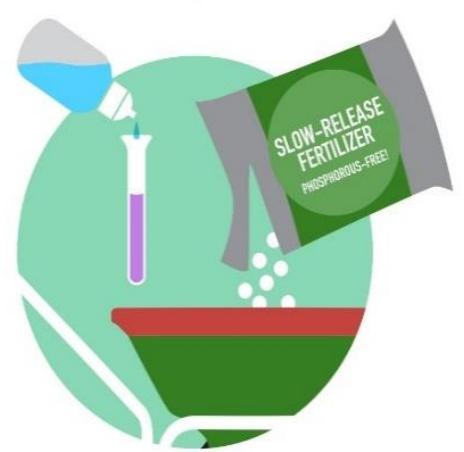

FERTILIZE PROPERLY

Fertilize based on a soil test, and use slow-release, phosphorous-free fertilizer.

Remove fertilizer from driveways, walkways, and roads after application.

Leave buffers untreated around water bodies and wetlands.

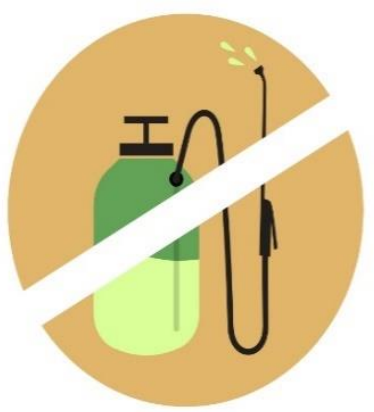

SAFER PESTICIDES

Use granular pesticides to reduce airborne particles and human exposure.

Granular pesticides pose less risk of runoff or leaching. 
Q35

If you would like to know more about the lawn care practices commonly adopted by green-certified lawn care, here are a few reputable sources*:

University of Rhode Island's Stormwater Solutions:

web.uri.edu/riss/take-action/simple-steps-at-home/around-the-yard/\#FertilizeSparingly University of Connecticut's Sustainable Landscaping:

http://www.sustainability.uconn.edu/sustain/turf/intro.html

University of Massachusetts' Turf Program:

https://ag.umass.edu/turf/publications-resources/best-management-practices

If you decide to check these websites out now, please click the above links and come back to the survey within a few minutes to complete.

*Please note that the best management practices on the websites above are examples of suggested practices. Each certified company chooses a set of practices to get greencertified and may not all adopt the same practice.

Did you check one or more of those websites?

O Yes, I did. (1)

O No, I did not. (2) 
Q36

In your opinion, which of the following lawn care practices do you think is effective in sustaining water quality? (Choose all that apply)

Conduct a soil test (1)

Maintain a lawn height of 3-4 inches (2)

Fertilize properly using non-phosphorous, slow-release fertilizer (3)

Clean up fertilizer from driveways, walkways, and roads after application (4)

Use safer pesticides and do not spread more than needed (5)

Leave areas untreated around water bodies and wetlands (6)

None the above (7) 
Q37

Were you aware that Rhode Island has a Green Certification program for lawn care companies?

○ Yes (1)

No (2) 


\section{Display This Question:}

If Who primarily maintains your lawn? (Please choose one) Lawn care company Is Selected

And How much did your household spend to hire the lawn care company in 2016? (In US dollars)\&nbsp; \&nbsp;(Please enter a NUMBER in the box.) \$ Is Not Empty Q38

There are several green-certified lawn care companies that serve your area. If you were to hire a green certified lawn care company to manage your lawn in 2017, how much would you be willing to pay for their services for the entire season?

You stated that you paid $\$ x$ for your services in 2016. Please use your payment for lawn care in 2016 as a reference.

Imagine that the green-certified lawn care companies will offer you the same services as the company you hired in 2016, but use best management practices that minimize the impact on water quality. Examples include:

- maintain a lawn height of 3-4 inches

- water efficiently

- fertilize properly using non-phosphorous, slow-release fertilizer

- use safer pesticides and do not spread more than needed. 


\section{Display This Question:}

If Who primarily maintains your lawn? (Please choose one) Lawn care company Is Not Selected

And Who primarily maintains your lawn? (Please choose one) Landlord/Complex management Is Not Selected

And How much did your household or the person managing your law spend in TOTAL in 2016 for your lawn care? (In US Dollars) Is Not Empty

And How many hours per month on average did your household or the person managing your ... Is Not Empty

Q39

There are several green-certified lawn care companies that serve your area. If you were to hire a green certified lawn care company to manage your lawn in 2017, how much would you be willing to pay for these services for the entire season?

Imagine that a green-certified company will offer you the following eco-friendly services:

- Five applications of fertilizer from early spring to late fall, at a proper rate, to avoid over fertilizing

- Use organic, slow-release fertilizer with micronutrients

- Broad-leaf weed and crabgrass control

- Pelletized limestone (early fall)

- Removal of materials from walks \& drives to minimize runoff.

For your information, you stated that your household spent $\$ \mathrm{x}$ annually and $\mathrm{x}$ hour(s) each month on average to take care of your lawn in 2016. Please use these figures as a reference. 


\section{Display This Question:}

If Who primarily maintains your lawn? (Please choose one) Lawn care company Is Selected

And How much did your household spend to hire the lawn care company in 2016? (In US dollars)\&nbsp; \&nbsp;(Please enter a NUMBER in the box.) \$ Is Empty $Q 40$

There are several green-certified lawn care companies that serve your area. If you were to hire a green certified lawn care company to manage your lawn in 2017 , how much would you be willing to pay for their services for the entire season?

Imagine that the green-certified lawn care companies will offer you the same services as the company you hired in 2016, but use best management practices that minimize the impact on water quality. Examples include:

- maintain a lawn height of 3-4 inches

-water efficiently

- fertilize properly using non-phosphorous, slow-release fertilizer

- use safer pesticides and do not spread more than needed. 


\section{Display This Question:}

If Who primarily maintains your lawn? (Please choose one) Lawn care company Is Not Selected

And Who primarily maintains your lawn? (Please choose one) Landlord/Complex management Is Not Selected

And How much did your household or the person managing your lawn spend in TOTAL in 2016 for your lawn care... \$ Is Empty

Q41

There are several green-certified lawn care companies that serve your area. If you were to hire a green certified lawn care company to manage your lawn in 2017, how much would you be willing to pay for these services for the entire season?

Imagine that a green-certified company will offer you the following eco-friendly services: - Five applications of fertilizer from early spring to late fall, at a proper rate to avoid over fertilizing

- Use organic, slow-release fertilizer with micronutrients

- Broad-leaf weed and crabgrass control

- Pelletized limestone (early fall)

- Removal of materials from walks \& drives to minimize runoff. 


\section{Display This Question:}

If Who primarily maintains your lawn? (Please choose one) Lawn care company Is Not Selected

And Who primarily maintains your lawn? (Please choose one) Landlord/Complex management Is Not Selected

And There are several green-certified lawn care companies that serve your area. If you were to hire...Is Not Displayed

And How many hours per month o... Is Empty

$Q 42$

There are several green-certified lawn care companies that serve your area. If you were to hire a green certified lawn care company to manage your lawn in 2017, how much would you be willing to pay for these services for the entire season?

Imagine that a green-certified company will offer you the following eco-friendly services:

- Five applications of fertilizer from early spring to late fall, at a proper rate, to avoid over fertilizing

- Use organic, slow-release fertilizer with micronutrients

- Broad-leaf weed and crabgrass control

- Pelletized limestone (early fall)

- Removal of materials from walks \& drives to minimize runoff. 
Information Treatment $3(Q 43-Q 58)$

Q43

Part II---Section 1: Your lawn care and water quality

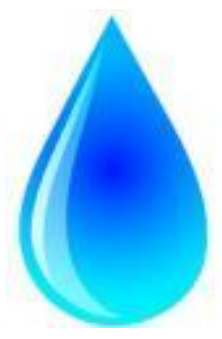

Here you will find information about how your lawn care can affect local water quality depending on how you manage it. Please read the information carefully. We will ask you one question following the information. 
$Q 44$

\section{WHAT DOES YOUR

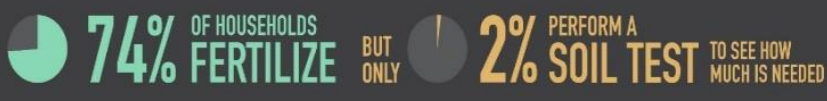
FERTILIZER DO?

A green lawn may look nice, but too much

fertilizer can have unintended consequences.

Proper lawn care is crucial in avoiding this!

SO YOU AND YOUR NEIGHBORS MAY JUST BE USING TOO MUCH FERTILIZER! TO MAKE THINGS WORSE:

$140 \%$ iss:

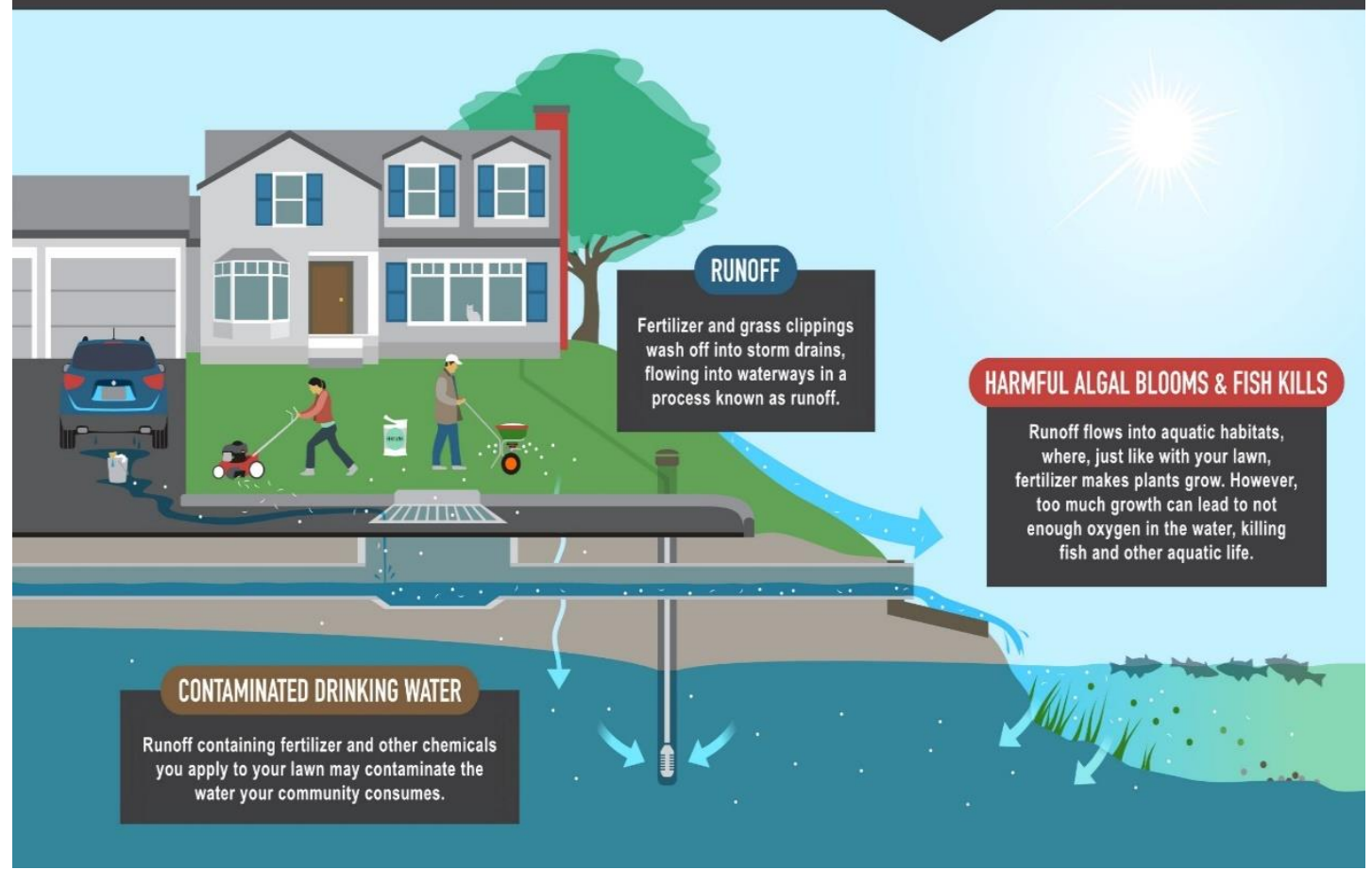

Data Sources:

https://cfpub.epa.gov/npstbx/files/Norming_Survey_LawnCare_NewEngland_July201 $\underline{0 . p d f}$

http://www.stormwatercenter.net/Practice/131-Homeowner\%20Survey.pdf 
Q45

In your opinion, which message from the previous figure was the most surprising to you? (Please choose one)

Improper fertilizer can be harmful to our drinking water, fish and other aquatic life. (1)

Proper lawn care can make a difference in protecting our drinking water, fish and other aquatic life. (2)

74\% of households fertilize their lawns. (3)

Only $2 \%$ of households conduct a soil test before fertilizing their lawns. (4)

$40 \%$ of the households use the whole bag of fertilizer to avoid storage. (5)

None of the above were surprising to me. (6) 
Q46

Part II---Section 2: About Green Certified Lawn Care Companies

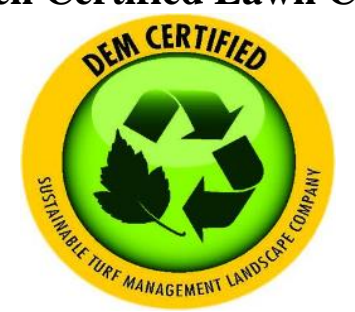

With proper care, it is possible to have a sustainable, responsible and healthy lawn.

In this section, you will find important information about services provided by 'greencertified' lawn care companies. Please read the information carefully. We will ask a few questions following the information. 
Q47

Since 2015, Rhode Island (RI) has a "Green Certification" program for lawn care companies. As of January 2017, 10 lawn care companies are green-certified by the RI Department of Environmental Management.

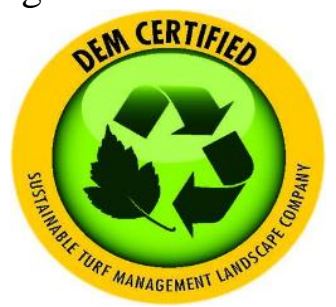

To be certified, lawn care companies must fulfill selected best management practices to protect water quality, conserve water, and contribute to the sustainability of the landscape.

Here are some practices the green-certified companies have adopted:

- mow appropriately (maintain a lawn height of 3-4 inches),

- fertilize appropriately (e.g., fertilize based on a soil test, use non-phosphorous, slow-release fertilizer),

- use safer pesticides (e.g., use mostly granular pesticides to reduce product drift and customer exposure) etc. when maintaining lawns.

Green-certified lawn care companies need to adopt additional best management practices to get re-certified every two years.

The NEXT graphic summarizes these practices. 
Q48

Here is a summary of what GREEN-CERTIFIED lawn care companies do.

CERTIFIED LAWN CARE COMPANIES MUST FULFILL BEST MANAGEMENT PRACTICES TO PROTECT WATER QUALITY:

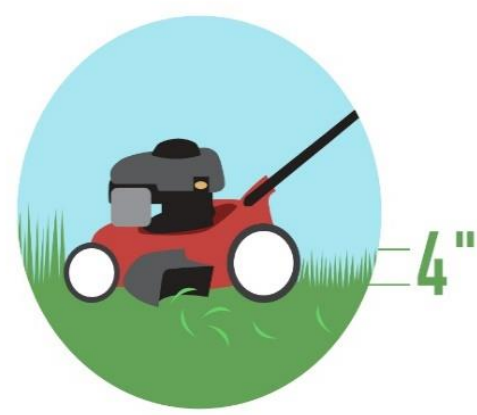

MOW PROPERLY

Maintain a lawn height of 3 to 4 inches.

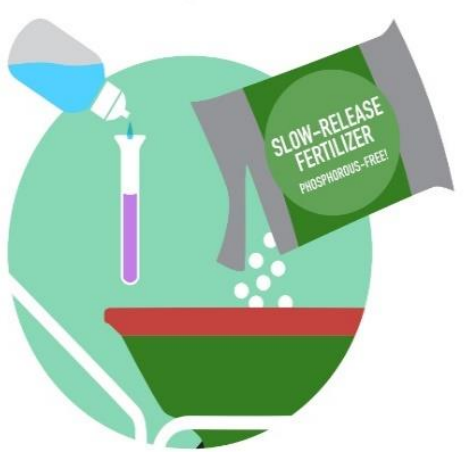

FERTILIZE PROPERLY

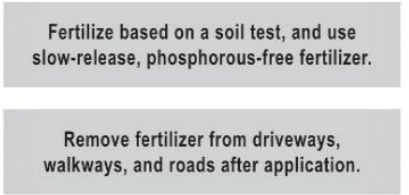

Leave buffers untreated around water bodies and wetlands.

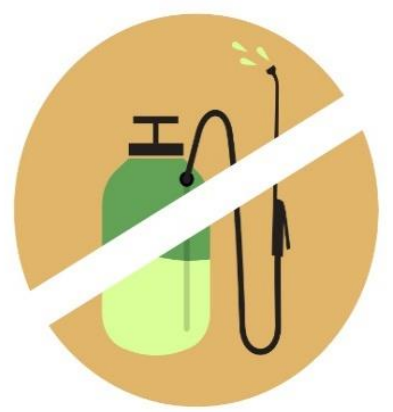

\section{SAFER PESTICIDES}

Use granular pesticides to reduce airborne particles and human exposure.

Granular pesticides pose less risk of runoff or leaching. 
Q49

If you would like to know more about the lawn care practices commonly adopted by green-certified lawn care, here are a few reputable sources*:

University of Rhode Island's Stormwater Solutions:

web.uri.edu/riss/take-action/simple-steps-at-home/around-the-yard/\#FertilizeSparingly University of Connecticut's Sustainable Landscaping:

http://www.sustainability.uconn.edu/sustain/turf/intro.html

University of Massachusetts' Turf Program:

https://ag.umass.edu/turf/publications-resources/best-management-practices

If you decide to check these websites out now, please click the above links and come back to the survey within a few minutes to complete.

*Please note that the best management practices on the websites above are examples of suggested practices. Each certified company chooses a set of practices to get greencertified and may not all adopt the same practice.

Did you check one or more of those websites?

O Yes, I did. (1)

O No, I did not. (2) 
Q50

In your opinion, which of the following lawn care practices do you think is effective in sustaining water quality? (Choose all that apply)

Conduct a soil test (1)

Maintain a lawn height of 3-4 inches (2)

Fertilize properly using non-phosphorous, slow-release fertilizer (3)

Clean up fertilizer from driveways, walkways, and roads after application (4)

Use safer pesticides and do not spread more needed (5)

Leave areas untreated around water bodies and wetlands (6)

․ None the above (7) 
Q51

Below is additional information about GREEN-CERTIFIED lawn care companies.

NEIGHBORS IN YOUR COMMUNITY HAVE STARTED TO HIRE GREEN-CERTIFIED LAWN CARE COMPANIES.

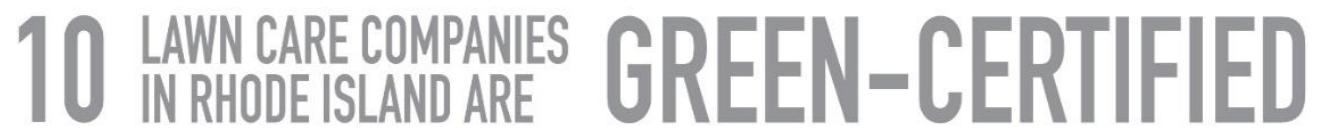

\section{$6000+$ RHODE ISLAND HIRED GREEN-CERTFEED LAWN}

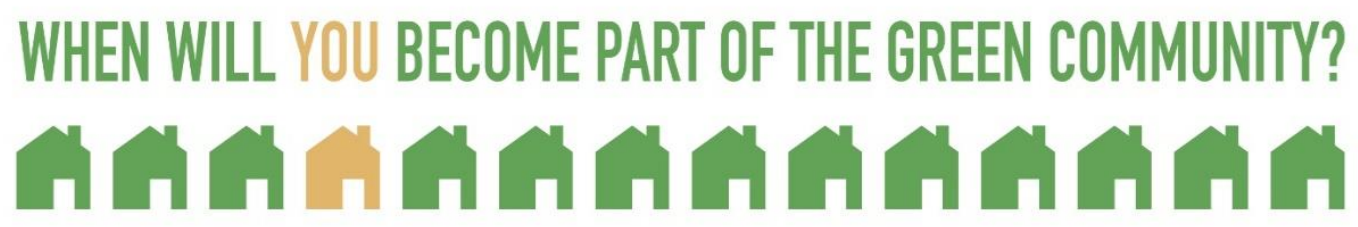


Q52

Based on the previous figure, how much do you agree with the following statement:

"Many residents in Rhode Island are already hiring Green Certified lawn care businesses."?

Extremely agree (1)

Moderately agree (2)

Slightly agree (3)

Slightly disagree (4)

Moderately disagree (5)

Extremely disagree (6) 
Q53

Were you aware that Rhode Island has a Green Certification program for lawn care companies?

○ Yes (1)

No (2) 


\section{Display This Question:}

If Who primarily maintains your lawn? (Please choose one) Lawn care company Is Selected

And How much did your household spend to hire the lawn care company in 2016? (In US dollars)\&nbsp; \&nbsp;(Please enter a NUMBER in the box.) \$ Is Not Empty Q54

There are several green-certified lawn care companies that serve your area. If you were to hire a green certified lawn care company to manage your lawn in 2017, how much would you be willing to pay for their services for the entire season?

You stated that you paid $\$ x$ for your services in 2016. Please use your payment for lawn care in 2016 as a reference.

Imagine that the green-certified lawn care companies will offer you the same services as the company you hired in 2016, but use best management practices that minimize the impact on water quality. Examples include:

- maintain a lawn height of 3-4 inches

- water efficiently

- fertilize properly using non-phosphorous, slow-release fertilizer

- use safer pesticides and do not spread more than needed. 


\section{Display This Question:}

If Who primarily maintains your lawn? (Please choose one) Lawn care company Is Not Selected

And Who primarily maintains your lawn? (Please choose one) Landlord/Complex management Is Not Selected

And How much did your household or the person managing your law spend in TOTAL in 2016 for your lawn care? (In US Dollars) Is Not Empty

And How many hours per month on average did your household or the person managing your ... Is Not Empty

Q55

There are several green-certified lawn care companies that serve your area. If you were to hire a green certified lawn care company to manage your lawn in 2017, how much would you be willing to pay for these services for the entire season?

Imagine that a green-certified company will offer you the following eco-friendly services:

- Five applications of fertilizer from early spring to late fall, at a proper rate, to avoid over fertilizing

- Use organic, slow-release fertilizer with micronutrients

- Broad-leaf weed and crabgrass control

- Pelletized limestone (early fall)

- Removal of materials from walks \& drives to minimize runoff.

For your information, you stated that your household spent $\$ \mathrm{x}$ annually and $\mathrm{x}$ hour(s) each month on average to take care of your lawn in 2016. Please use these figures as a reference. 


\section{Display This Question:}

If Who primarily maintains your lawn? (Please choose one) Lawn care company Is Selected

And How much did your household spend to hire the lawn care company in 2016? (In US dollars)\&nbsp; \&nbsp;(Please enter a NUMBER in the box.) \$ Is Empty Q56

There are several green-certified lawn care companies that serve your area. If you were to hire a green certified lawn care company to manage your lawn in 2017, how much would you be willing to pay for their services for the entire season?

Imagine that the green-certified lawn care companies will offer you the same services as the company you hired in 2016, but use best management practices that minimize the impact on water quality. Examples include:

- maintain a lawn height of 3-4 inches

-water efficiently

- fertilize properly using non-phosphorous, slow-release fertilizer

- use safer pesticides and do not spread more than needed. 


\section{Display This Question:}

If Who primarily maintains your lawn? (Please choose one) Lawn care company Is Not Selected

And Who primarily maintains your lawn? (Please choose one) Landlord/Complex management Is Not Selected

And How much did your household or the person managing your lawn spend in TOTAL in 2016 for your lawn care... \$ Is Empty

Q57

There are several green-certified lawn care companies that serve your area. If you were to hire a green certified lawn care company to manage your lawn in 2017, how much would you be willing to pay for these services for the entire season?

Imagine that a green-certified company will offer you the following eco-friendly services: - Five applications of fertilizer from early spring to late fall, at a proper rate to avoid over fertilizing

- Use organic, slow-release fertilizer with micronutrients

- Broad-leaf weed and crabgrass control

- Pelletized limestone (early fall)

- Removal of materials from walks \& drives to minimize runoff. 


\section{Display This Question:}

If Who primarily maintains your lawn? (Please choose one) Lawn care company Is Not Selected

And Who primarily maintains your lawn? (Please choose one) Landlord/Complex management Is Not Selected

And There are several green-certified lawn care companies that serve your area. If you were to hire...Is Not Displayed

And How many hours per month o... Is Empty Q58

There are several green-certified lawn care companies that serve your area. If you were to hire a green certified lawn care company to manage your lawn in 2017, how much would you be willing to pay for these services for the entire season?

Imagine that a green-certified company will offer you the following eco-friendly services:

- Five applications of fertilizer from early spring to late fall, at a proper rate, to avoid over fertilizing

- Use organic, slow-release fertilizer with micronutrients

- Broad-leaf weed and crabgrass control

- Pelletized limestone (early fall)

- Removal of materials from walks \& drives to minimize runoff. 
One of the following four treatments will be randomly displayed at the same probability for each survey. (The second treatment and the third treatment are identical.)

Financial incentive Treatment 1: no incentive (Q59-Q60)

Q59

Given the information provided to you, how likely are you going to hire a greencertified lawn care company to manage your lawn? (Please choose one)

O Extremely likely (1)

O Moderately likely (2)

O Slightly likely (3)

O Slightly unlikely (4)

O Moderately unlikely (5)

O Extremely unlikely (6) 
Q60

What factors would affect your decision to contract into a green-certified lawn care company? (Choose all that apply)

How much it costs annually (1)

The type of services provided (e.g., mowing, watering, fertilizing, etc.) (2)

- Number of visits for treatment (3)

Type of environmentally-friendly practices (4)

Other [please specify] (5) 
Financial incentive Treatment 2: $25 \%$ of contract (Q61-Q64)

Q61

Congratulations!

You have been randomly selected to receive a rebate of $\mathbf{2 5 \%}$ of your first year's annual contract fee with a green-certified lawn care company (up to $\$ 75$ ). The rebate is sponsored by a research grant.

Don't miss this opportunity---this limited offer is valid only for contracts signed by May 31, 2017.

This is an actual rebate offer for you. If you contract with one of the three green-certified lawn care companies listed at the end of this survey, you will receive the rebate.

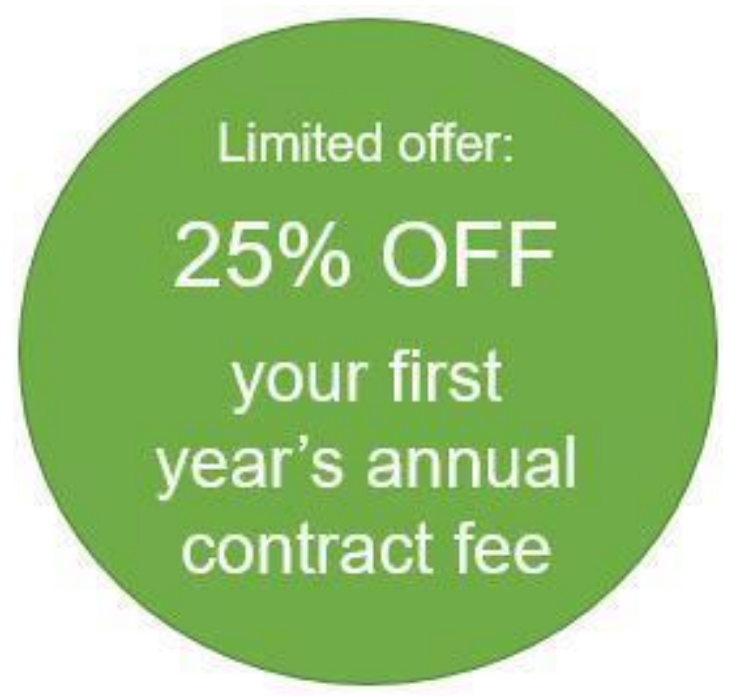


Q62

How important is the rebate to your decision?

O Extremely important (1)

O Moderately important (2)

O Slightly important (3)

O Slightly unimportant (4)

O Moderately unimportant (5)

O Extremely unimportant (6) 
Q63

Given the information and rebate provided to you, how likely are you going to hire a green-certified lawn care company to manage your lawn? (Please choose one) Note: This is an actual rebate offer for you. If you contract with one of the three green-certified lawn care companies listed at the end of this survey, you will receive the rebate.

O Extremely likely (1)

O Moderately likely (2)

O Slightly likely (3)

O Slightly unlikely (4)

O Moderately unlikely (5)

O Extremely unlikely (6) 
Q64

What factors would affect your decision to contract into a green-certified lawn care company? (Choose all that apply)

- How much it costs annually (1)

The type of services provided (e.g., mowing, watering, fertilizing, etc.) (2)

- Number of visits for treatment (3)

Type of environmentally-friendly practices (4)

Other [please specify] (5) 
Financial incentive Treatment 3: $25 \%$ of contract (Q65-Q68)

Q65

Congratulations!

You have been randomly selected to receive a rebate of $\mathbf{2 5 \%}$ of your first year's annual contract fee with a green-certified lawn care company (up to $\$ 75$ ). The rebate is sponsored by a research grant.

Don't miss this opportunity---this limited offer is valid only for contracts signed by May 31, 2017.

This is an actual rebate offer for you. If you contract with one of the three green-certified lawn care companies listed at the end of this survey, you will receive the rebate.

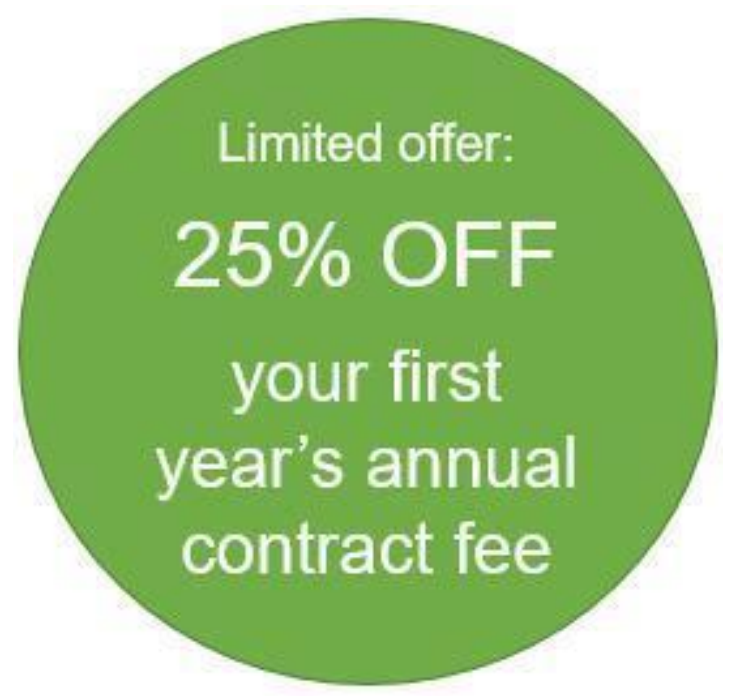


Q66

How important is the rebate to your decision?

O Extremely important (1)

O Moderately important (2)

O Slightly important (3)

O Slightly unimportant (4)

O Moderately unimportant (5)

O Extremely unimportant (6) 
Q67

Given the information and rebate provided to you, how likely are you going to hire a green-certified lawn care company to manage your lawn? (Please choose one) Note: This is an actual rebate offer for you. If you contract with one of the three green-certified lawn care companies listed at the end of this survey, you will receive the rebate.

O Extremely likely (1)

O Moderately likely (2)

O Slightly likely (3)

O Slightly unlikely (4)

O Moderately unlikely (5)

O Extremely unlikely (6) 
Q68

What factors would affect your decision to contract into a green-certified lawn care company? (Choose all that apply)

- How much it costs annually (1)

The type of services provided (e.g., mowing, watering, fertilizing, etc.) (2)

- Number of visits for treatment (3)

Type of environmentally-friendly practices (4)

Other [please specify] (5) 
Financial incentive Treatment 4: 50\% of contract (Q69-Q72)

Q69

Congratulations!

You have been randomly selected to receive a rebate of $\mathbf{5 0 \%}$ of your first year's annual contract fee with a green-certified lawn care company (up to $\$ 150$ ). The rebate is sponsored by a research grant.

Don't miss this opportunity---this limited offer is valid only for contracts signed by May 31, 2017.

This is an actual rebate offer for you. If you contract with one of the three green-certified lawn care companies listed at the end of this survey, you will receive the rebate.

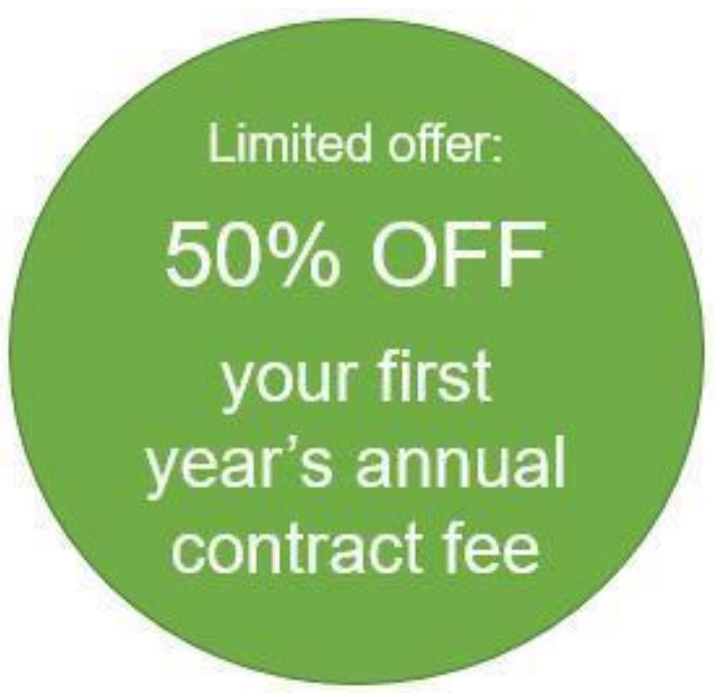


Q70

How important is the rebate to your decision?

O Extremely important (1)

O Moderately important (2)

O Slightly important (3)

O Slightly unimportant (4)

O Moderately unimportant (5)

O Extremely unimportant (6) 
Q71

Given the information and rebate provided to you, how likely are you going to hire a green-certified lawn care company to manage your lawn? (Please choose one) Note: This is an actual rebate offer for you. If you contract with one of the three green-certified lawn care companies listed at the end of this survey, you will receive the rebate.

O Extremely likely (1)

O Moderately likely (2)

O Slightly likely (3)

O Slightly unlikely (4)

O Moderately unlikely (5)

O Extremely unlikely (6) 
$Q 72$

What factors would affect your decision to contract into a green-certified lawn care company? (Choose all that apply)

- How much it costs annually (1)

The type of services provided (e.g., mowing, watering, fertilizing, etc.) (2)

Number of visits for treatment (3)

Type of environmentally-friendly practices (4)

Other [please specify] (5) 
Survey Part III: Contract (Q73-Q81)

$Q 73$

Part III: Contracting with a green-certified lawn care company

In this section, please tell us whether you are interested in getting more information about green-certified lawn care companies. 
$Q 74$

For this research project, the researchers at University of Rhode Island are collaborating with the following three green-certified lawn care companies (in alphabetical order):

A Cut Above (http://rilawncare.com/)

Dana Designs (http://danalandscapedesigns.com/)

SeaScape (http://seascapeinc.com/)

Are you interested in signing up here to receive more information about their services?

If you sign up, one or more of the three green-certified lawn care companies serving your area will contact you to give you more information, including a free estimate.

O Yes, I am interested in signing up. (1)

No, I am not interested at this time. (2) 


\section{Display This Question:}

If Would you be interested in getting lawn care services from one green certified lawn care company? Yes Is Selected

Q75 Please leave your name, phone number and email address below. One or more of the three green-certified lawn care companies serving your area will contact you to give you more information.

Display This Question:

If Would you be interested in getting lawn care services from one green certified lawn care company? Yes Is Selected

Q76 Your first name:

\section{Display This Question:}

If The researchers at the University of Rhode Island are collaborating with three green-certified lawn c... Yes, I am interested in obtaining more information. Is Selected Q77 Your last name:

\section{Display This Question:}

If Would you be interested in getting lawn care services from one green certified lawn care company? Yes Is Selected

Q78 Your phone number (please include the three-digit area code):

\section{Display This Question:}

If Would you be interested in getting lawn care services from one green certified lawn care company? <o:p $\rangle\langle/ o: p>$ Yes Is Selected

Q79 Your email address:

\section{Display This Question:}

If The researchers at the University of Rhode Island are collaborating with three green-certified lawn c... Yes, I am interested in obtaining more information. Is Selected $Q 80$ Please re-type your email address here to verify it. 


\section{Display This Question:}

If The researchers at the University of Rhode Island are collaborating with three green-certified lawn c... No Is Selected

Q81

Would you mind telling us why you are not interested in signing up to get information from green-certified lawn care companies? (Choose all that apply)

I I do not want to change from my current contractor. (1)

I I already have a good relationship with my current contractor. (2)

I I do not think practices by green certified lawn care companies help improve water quality. (3)

I do not want to give away my personal information. (4)

- Hiring a lawn care professional is too expensive. (5)

I I prefer to managing my lawn by myself. (6)

O Other [Please specify] (7) 
Survey Part IV (Q82-Q96)

Q82

Part IV: A bit about you

In this section, we will ask a few questions about you and your household. 
Q83

What is your gender?

O Male (1)

Female (2)

Prefer not to answer (3) 
Q84

What is your age?

O 18-24 years old (1)

O 25-34 years old (2)

O 35-44 years old (3)

O 45-54 years old (4)

O 55-64 years old (5)

O 65-74 years old (6)

O 75 years or older (7) 
Q85

Which of the following best describes the highest level of education you have completed?

Less than 12 years, no high school diploma (1)

High School (2)

O GED (3)

O Some college (4)

Trade Certificate (5)

O Bachelor's Degree (6)

Master's degree or higher (7) 
Q86

What was your household's total income (before taxes) in 2016?

Less than $\$ 20,000$ (1)

O $\$ 20,000$ to $\$ 39,999$ (2)

○ $\$ 40,000$ to $\$ 59,999$ (3)

O $\$ 60,000$ to $\$ 79,999$ (4)

O $\$ 80,000$ to $\$ 99,999$ (5)

O $\$ 100,000$ to $\$ 149,999$ (6)

O $\$ 150,000$ or more (7) 


\begin{tabular}{|c|c|c|c|c|}
\hline $\begin{array}{l}\text { Q87 Please rate the } \\
\text { effect of the } \\
\text { following } \\
\text { interventions to } \\
\text { improve water } \\
\text { quality in your area. }\end{array}$ & $\begin{array}{l}\text { Very } \\
\text { effective (1) }\end{array}$ & Effective (2) & $\begin{array}{l}\text { Somewhat } \\
\text { effective (3) }\end{array}$ & $\begin{array}{l}\text { Not effective } \\
\text { (4) }\end{array}$ \\
\hline $\begin{array}{l}\text { Control industrial } \\
\text { pollution }\end{array}$ & 0 & $\mathrm{O}$ & 0 & $\mathrm{O}$ \\
\hline $\begin{array}{ll}\text { Use } & \text { best } \\
\text { management } \\
\text { practices for lawn } \\
\text { care }\end{array}$ & 0 & $\mathrm{O}$ & $\mathrm{O}$ & $\mathrm{O}$ \\
\hline $\begin{array}{l}\text { Control agricultural } \\
\text { pollution }\end{array}$ & $\mathrm{O}$ & $\mathrm{O}$ & $\mathrm{O}$ & $\mathrm{O}$ \\
\hline Clean pet waste & O & $\mathrm{O}$ & O & O \\
\hline $\begin{array}{l}\text { Manage } \quad \text { sewage } \\
\text { pollution }\end{array}$ & 0 & $\mathrm{O}$ & $\mathrm{O}$ & O \\
\hline $\begin{array}{l}\text { Manage stormwater } \\
\text { pollution }\end{array}$ & $\mathrm{O}$ & $\mathrm{O}$ & 0 & O \\
\hline
\end{tabular}




\begin{tabular}{|c|c|c|c|c|c|c|c|}
\hline $\begin{array}{l}\text { Q88 Please } \\
\text { indicate } \\
\text { how } \\
\text { strongly } \\
\text { you agree } \\
\text { or disagree } \\
\text { with each } \\
\text { of the } \\
\text { following } \\
\text { statements. }\end{array}$ & $\begin{array}{l}\text { Complet } \\
\text { ely agree } \\
\text { (6) }\end{array}$ & $\begin{array}{l}\text { Agre } \\
\text { e (5) }\end{array}$ & $\begin{array}{l}\text { Somew } \\
\text { hat } \\
\text { agree } \\
\text { (4) }\end{array}$ & $\begin{array}{l}\text { Neithe } \\
\text { r agree } \\
\text { nor } \\
\text { disagr } \\
\text { ee (3) }\end{array}$ & $\begin{array}{l}\text { Somew } \\
\text { hat } \\
\text { disagree } \\
\text { (2) }\end{array}$ & $\begin{array}{l}\text { Disagr } \\
\text { ee (1) }\end{array}$ & $\begin{array}{l}\text { Complet } \\
\text { ely } \\
\text { disagree } \\
(0)\end{array}$ \\
\hline $\begin{array}{l}\text { We are } \\
\text { approachin } \\
\mathrm{g} \text { the limit } \\
\text { of the } \\
\text { number of } \\
\text { people the } \\
\text { earth can } \\
\text { support. }\end{array}$ & 0 & 0 & 0 & 0 & 0 & 0 & 0 \\
\hline $\begin{array}{l}\text { Humans } \\
\text { have the } \\
\text { right to } \\
\text { modify the } \\
\text { natural } \\
\text { environme } \\
\text { nt to suit } \\
\text { their needs. }\end{array}$ & 0 & 0 & 0 & 0 & 0 & 0 & 0 \\
\hline $\begin{array}{l}\text { When } \\
\text { humans } \\
\text { interfere } \\
\text { with nature } \\
\text { it often } \\
\text { produces } \\
\text { disastrous } \\
\text { consequenc } \\
\text { es. }\end{array}$ & 0 & 0 & 0 & 0 & 0 & 0 & 0 \\
\hline $\begin{array}{l}\text { Human } \\
\text { ingenuity } \\
\text { will insure } \\
\text { that we do } \\
\text { not make } \\
\text { the Earth } \\
\text { unlivable. }\end{array}$ & 0 & 0 & 0 & 0 & 0 & 0 & 0 \\
\hline
\end{tabular}




\begin{tabular}{|c|c|c|c|c|c|c|c|}
\hline $\begin{array}{l}\text { Humans are } \\
\text { seriously } \\
\text { abusing the } \\
\text { environme } \\
\text { nt. }\end{array}$ & 0 & 0 & 0 & 0 & 0 & 0 & 0 \\
\hline $\begin{array}{l}\text { The Earth } \\
\text { has plenty } \\
\text { of natural } \\
\text { resources if } \\
\text { we just } \\
\text { learn how } \\
\text { to develop } \\
\text { them. }\end{array}$ & $\bigcirc$ & $\bigcirc$ & 0 & 0 & 0 & 0 & 0 \\
\hline $\begin{array}{l}\text { Plants and } \\
\text { animals } \\
\text { have as } \\
\text { much right } \\
\text { as humans } \\
\text { to exist. }\end{array}$ & 0 & 0 & 0 & $\bigcirc$ & 0 & 0 & $\bigcirc$ \\
\hline
\end{tabular}




\begin{tabular}{|c|c|c|c|c|c|c|c|}
\hline $\begin{array}{l}\text { Q89 } \\
\text { Please } \\
\text { indicate } \\
\text { how } \\
\text { strongly } \\
\text { you agree } \\
\text { or } \\
\text { disagree } \\
\text { with each } \\
\text { of the } \\
\text { following } \\
\text { statements }\end{array}$ & $\begin{array}{l}\text { Complete } \\
\text { ly agree } \\
(6)\end{array}$ & $\begin{array}{l}\text { Agre } \\
\text { e (5) }\end{array}$ & $\begin{array}{l}\text { Somewh } \\
\text { at agree } \\
\text { (4) }\end{array}$ & $\begin{array}{l}\text { Neithe } \\
\mathrm{r} \text { agree } \\
\text { nor } \\
\text { disagr } \\
\text { ee }(3)\end{array}$ & $\begin{array}{l}\text { Somewh } \\
\text { at } \\
\text { disagree } \\
\text { (2) }\end{array}$ & $\begin{array}{l}\text { Disagr } \\
\text { ee }(1)\end{array}$ & $\begin{array}{l}\text { Complete } \\
\text { ly } \\
\text { disagree } \\
(0)\end{array}$ \\
\hline $\begin{array}{l}\text { The } \\
\text { balance of } \\
\text { nature is } \\
\text { strong } \\
\text { enough to } \\
\text { cope with } \\
\text { the } \\
\text { impacts of } \\
\text { modern } \\
\text { industrial } \\
\text { nations. }\end{array}$ & 0 & 0 & 0 & 0 & 0 & 0 & 0 \\
\hline $\begin{array}{l}\text { Despite } \\
\text { our } \\
\text { special } \\
\text { abilities, } \\
\text {, } \\
\text { humans } \\
\text { are still } \\
\text { subject to } \\
\text { the laws of } \\
\text { nature. }\end{array}$ & 0 & 0 & 0 & 0 & 0 & 0 & 0 \\
\hline $\begin{array}{l}\text { The so- } \\
\text { called } \\
\text { "ecologica } \\
1 \text { crisis" } \\
\text { facing } \\
\text { humankin } \\
\text { d has been } \\
\text { greatly } \\
\text { exaggerat } \\
\text { ed. }\end{array}$ & 0 & 0 & 0 & 0 & 0 & 0 & 0 \\
\hline
\end{tabular}




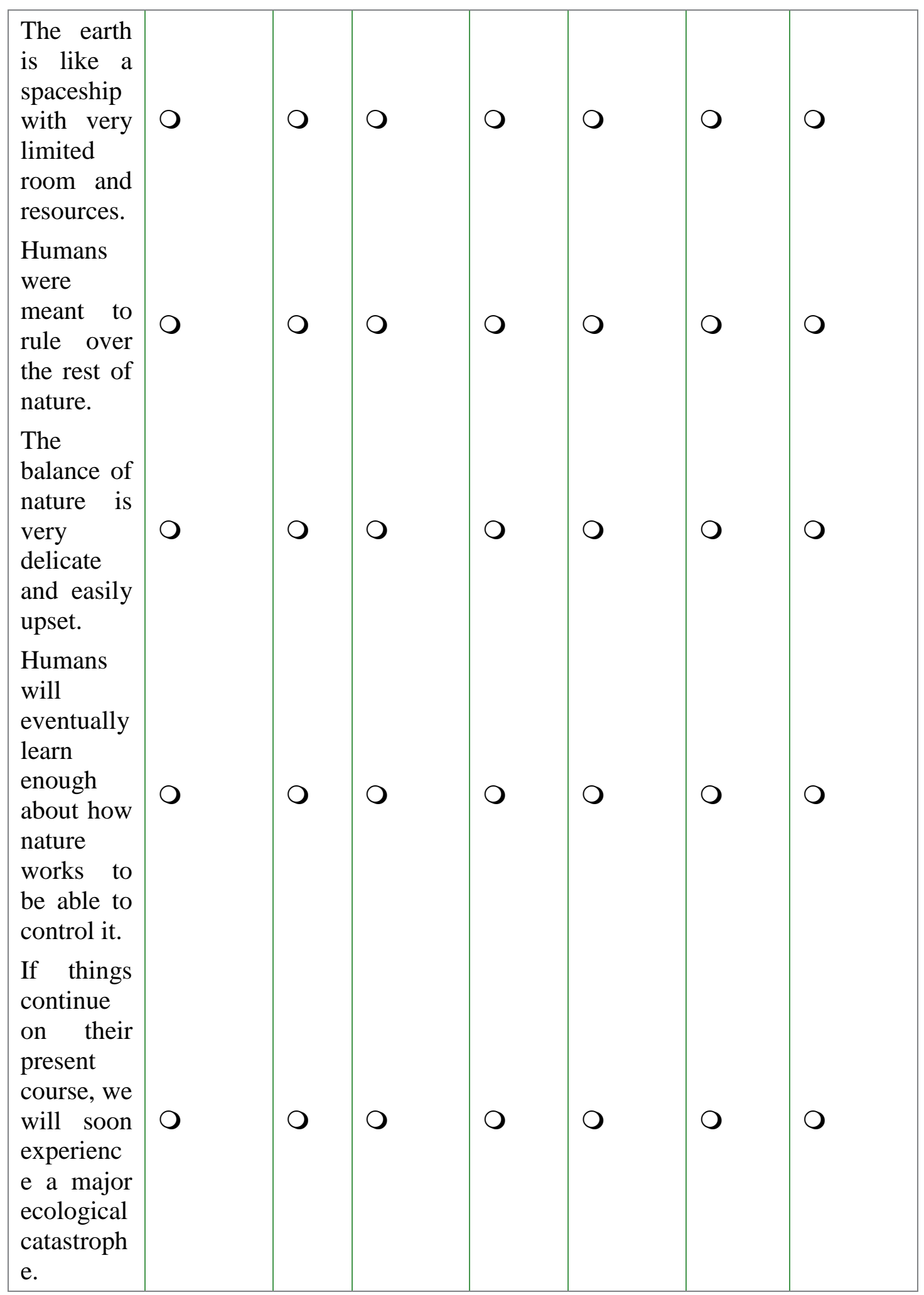




\begin{tabular}{|c|c|c|c|c|c|}
\hline $\begin{array}{l}\text { Q90 Check the } \\
\text { category on the } \\
\text { right that conforms } \\
\text { to the frequency } \\
\text { with which you } \\
\text { have carried out } \\
\text { the following acts. }\end{array}$ & Never (0) & Once (1) & $\begin{array}{l}\text { More than } \\
\text { once (2) }\end{array}$ & Often (3) & $\begin{array}{l}\text { Very } \\
\text { often (4) }\end{array}$ \\
\hline $\begin{array}{l}\text { I have helped push } \\
\text { a stranger's car out } \\
\text { of the snow. (1) }\end{array}$ & 0 & 0 & 0 & O & 0 \\
\hline $\begin{array}{l}\text { I have given } \\
\text { directions to a } \\
\text { stranger. (2) }\end{array}$ & 0 & 0 & 0 & O & O \\
\hline $\begin{array}{l}\text { I have made } \\
\text { change for a } \\
\text { stranger. (3) }\end{array}$ & 0 & 0 & 0 & 0 & O \\
\hline $\begin{array}{l}\text { I have given } \\
\text { money to a charity. } \\
\text { (4) }\end{array}$ & O & O & O & O & 0 \\
\hline $\begin{array}{l}\text { I have given } \\
\text { money to a } \\
\text { stranger who } \\
\text { needed it (or asked } \\
\text { me for it). (5) }\end{array}$ & 0 & 0 & 0 & 0 & 0 \\
\hline $\begin{array}{l}\text { I have donated } \\
\text { goods or clothes to } \\
\text { a charity. (6) }\end{array}$ & 0 & 0 & 0 & 0 & O \\
\hline $\begin{array}{l}\text { I have done } \\
\text { volunteer work for } \\
\text { a charity. (7) }\end{array}$ & 0 & 0 & 0 & $O$ & 0 \\
\hline $\begin{array}{l}\text { I have donated } \\
\text { blood. (8) }\end{array}$ & 0 & 0 & 0 & 0 & O \\
\hline $\begin{array}{l}\text { I have helped carry } \\
\text { a stranger's } \\
\text { belongings (books, } \\
\text { parcels, etc.). (9) }\end{array}$ & 0 & 0 & 0 & 0 & O \\
\hline $\begin{array}{l}\text { I have delayed an } \\
\text { elevator and held } \\
\text { the door open for a } \\
\text { stranger. (10) }\end{array}$ & 0 & 0 & 0 & 0 & 0 \\
\hline
\end{tabular}




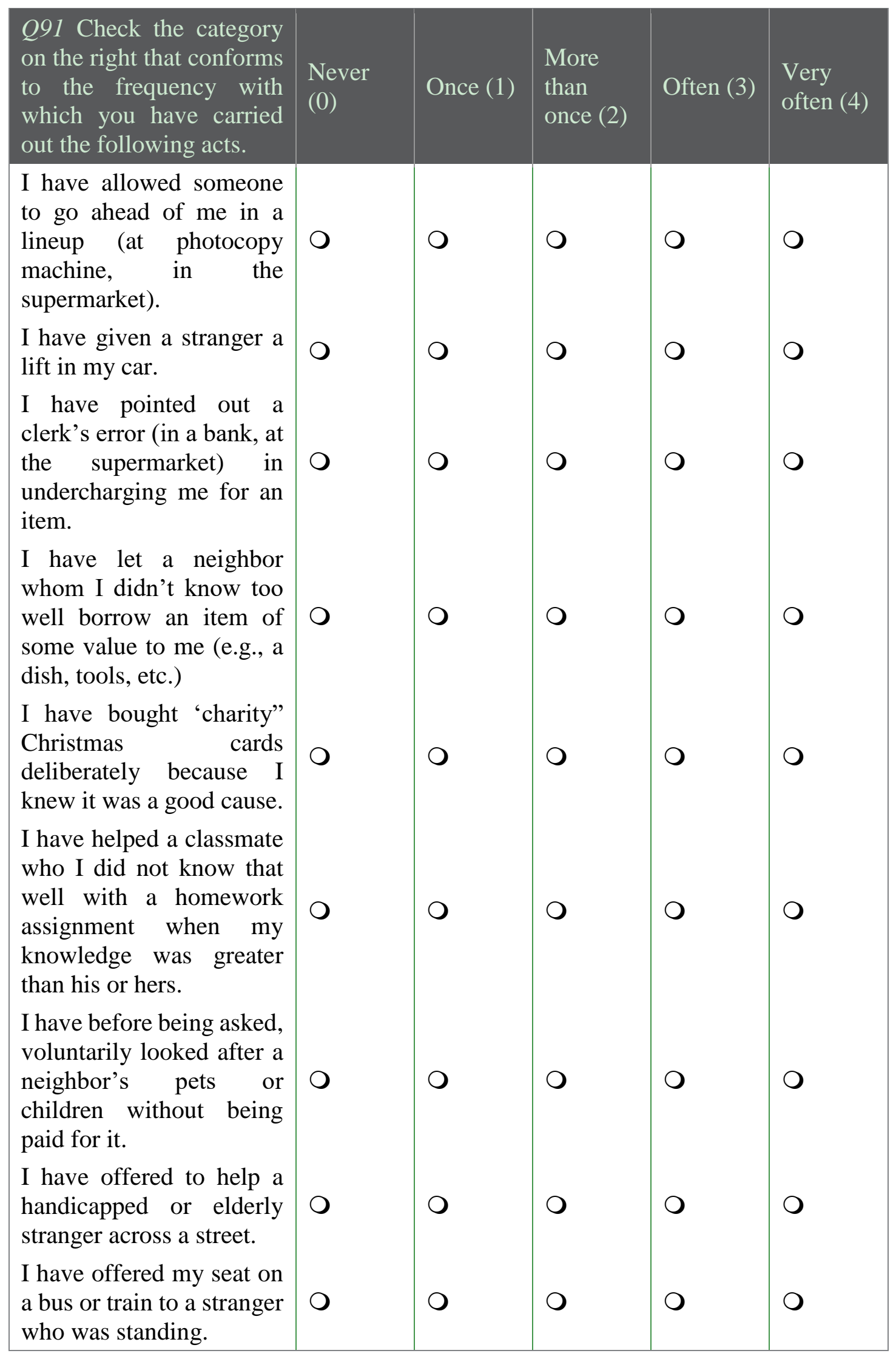




\begin{tabular}{|l|l|l|l|l|l|}
\hline $\begin{array}{l}\text { I have helped an } \\
\text { acquaintance to move } \\
\text { households. (20) }\end{array}$ & $\bigcirc$ & 0 & 0 & 0 & 0 \\
\hline
\end{tabular}

\section{Display This Question:}

If Please re-type your email here to verify it. Text Response Is Empty

Q92 As a small gift, we would like to send you a \$2 Amazon e-gift card. You can either use it yourself or give it to someone by simply forwarding the email you will receive from Amazon.com. For example, you can donate to your child's school or other organizations. Please leave your email address below. By typing your email below, you give us permission to send your email to amazon.com. Make sure your email address is typed correctly.

You can also forgo the Amazon e-gift card by simply leaving the following space blank.

\section{Display This Question:}

If Please re-type your email here to verify it. Text Response Is Not Empty

Q93 As a small gift, we would like to send you a \$2 Amazon e-gift card to the email address you provided in Part III. You can either use it yourself or give it to someone by simply forwarding the email. For example, you can donate to your child's school or other organizations. Would you like a \$2 Amazon e-gift card? By choosing "Yes", you give us permission to send your email to amazon.com.

O Yes, please email me the e-gift card. (2)

O No, thanks. (3) 


\section{Display This Question:}

If As a small gift, we would like to send you a $\$ 2$ Amazon e-gift card. Please leave your email addre... Text Response Is Not Empty

And As a small gift, we would like to send you a $\$ 2$ Amazon e-gift card. Please leave your email addre... Text Response Is Displayed

Q94

Please re-type your email here to verify it. 


\section{Display This Question:}

If As a small gift, we would like to send you a \$2 Amazon e-gift card. You can either use it yours... Text Response Is Not Empty

Or As a small gift, we would like to send you a $\$ 2$ Amazon e-gift card to the email address you provi... Yes, please email me the e-gift card. Is Selected

Q95 We will email you a \$2 Amazon e-gift card to your email address. Please sign (type) your name below. With this signature, you assure us that:

1. You are the only person in your household who will take this survey.

2. You understand that you will receive a $\$ 2$ Amazon e-gift card within one week after you finish the survey.

3. You allow us to share your email address with amazon.com. 
Q96

Thank you for your time!

If you have additional comments, please indicate in the box below. 


\section{Appendix II: A list of organizations who distributed our survey}

\begin{tabular}{|c|}
\hline Quest School \\
\hline Westerly Public Schools \\
\hline Cranston Public Schools \\
\hline Compass School \\
\hline Kingston Hill Academy \\
\hline Aquidneck Land Trust \\
\hline Save the Bay \\
\hline Environment Justice League \\
\hline The Nature Conservancy \\
\hline Wood-Pawcatuck Watershed Association \\
\hline Woonasquatucket River Watershed Council \\
\hline URI Master Gardeners \\
\hline Northern Rhode Island Conservation District \\
\hline Eastern RI Conservation District \\
\hline RI Rivers Council \\
\hline Watershed Watch \\
\hline Blackstone River Watershed Association \\
\hline Briggs Farm Improvement Association \\
\hline
\end{tabular}


Appendix III: An example of recruitment advertisement

\begin{tabular}{|c|c|}
\hline $\begin{array}{c}\text { THINK BIG } \\
\text { WE DO' }\end{array}$ & $\begin{array}{c}\text { Passionate about water quality issues? } \\
\text { National Science Foundation funded survey } \\
\text { conducted by URI researchers will collect } \\
\text { information related to lawn care and water } \\
\text { quality. Please fill out by March } 31^{\text {st }} \\
\text { Participants receive } \$ 2 \text { Amazon credit. } \\
\text { We are counting on YOU }\end{array}$ \\
\hline
\end{tabular}




\section{Appendix IV: Experiment instructions}

\section{Appendix 4.1: Certainty treatments}

\section{Instructions (Part I)}

This experiment is a study of individual and group decision-making. If you follow these instructions carefully and make informed decisions, you will earn money. The money you earn will be paid to you, in cash, at the end of the experiment.

You will be in a group consisting of six players. Each player plays the role of a firm. Your firm and five other firms are located near a lake next to a town. Anyone in the town can access the lake to go fishing, swimming or boating.

Your firm makes money through its production. Production also generates emissions, which affect the water quality of the lake. In general, the higher your production the more you earn from production, but the greater the emissions your firm generates. Firm earnings are denominated in "experimental dollars", which will be exchanged for cash at the end of the experiment at the rate of 2150 experimental dollars to $\$ 1$.

This experiment is broken up into three parts and $\mathbf{1 0 0}$ decision "rounds", 45 rounds in Part I, 45rounds in Part II and 10 rounds in Part III. Each round is independent, meaning that decisions during a round do not affect future rounds in any way. In each round your task is to choose among six management options, labeled " $A$ " through " $F$ ". These options remain constant throughout the experiment. Associated with each management option is: (1) a production level (Production); (2) the emissions generated (Emissions); and (3) the number of experimental dollars you earn from product sales (Earnings from Production). Your final earnings at the end of the experiment is whatever you earn in the 100 rounds based on you and your group members' decisions, plus a show-up fee of $\$ 5$.

You have been provided the following table (Table 1) titled "Initial Earnings" that lists the emissions, levels of production, and earnings from production that are associated with each management option. Please refer to this table before making any decisions.

Table 1: Initial Earnings

\begin{tabular}{cccc}
\hline Management Option & Emissions & Production & $\begin{array}{l}\text { Earnings from production } \\
\text { (Experimental Dollars) }\end{array}$ \\
\hline A & 6 & 150 & 800 \\
B & 5 & 138 & 797 \\
C & 4 & 123 & 786 \\
D & 3 & 108 & 764 \\
E & 2 & 89 & 725 \\
F & 1 & 66 & 655 \\
\hline
\end{tabular}


Your firm's emissions will result in pollution in the lake. Pollution affects the wellbeing of all lake users. For example, high pollution levels affect the health of fish, causing losses to the fisherman. To protect the water resource, an environmental regulator requires you, and all other firms in your group, to pay the following Tax on pollution:

\section{Tax $=30 *$ Total Pollution}

Total Pollution equals the total of your firm's emissions and the other five players' emissions. In other words, the total pollution is based on the management decision of everyone in your group, not just your own.

A round of the experiment is complete when all six players have made their management decision by choosing from the six management options listed on your computer screen. After every player makes his or her management decision, Total Pollution will be calculated by the computer. The Tax will be calculated using the formula above. For each round, your Total Earnings is calculated as follows:

\section{Total Earnings = Earnings from Production - Tax}

Your computer will show your Management Options, your Earnings from Production, the Total Pollution, the Tax and your Total Earnings from the current round and all previous rounds. Your computer will also show you other five players' Management Options after everyone makes their decision in one round.

Here is an example of how to calculate your Total Earnings. If in one round each of five others in your group chooses option $\mathrm{E}$ and has an individual emission of 2 and you choose option A and have an individual emission of 6, the Total Pollution will be 16 . Everyone in your group will pay a tax that is equal to 480 experimental dollars. Your total earnings in this round will be $800-480=320$ experimental dollars.

Here is another example. If in one round all of you choose option A and each of you have an individual emission of 6, the Total Pollution will be 36. Everyone in your group will pay a tax that is equal to 1080 experimental dollars. Your total earnings in this round will be $800-1080=-220$ experimental dollars, which means you lose 220 experimental dollars.

It is important that you understand the instructions and the experiment. We will ask you a few questions before you make any decisions in the experiment. The computer will check your answers automatically. If you do not get the correct answers after a few attempts, please let the experiment administrator know and he or she will be happy to explain them to you. 


\section{Please do not turn to next page unless experimenters ask you to do so.}




\section{Instructions (Part II)}

You will make another 45 decisions in this part. Everything in the game will keep the same except that other firms' management option choices in your group will not be given to you anymore. Please also read the instructions in Part I to make any decisions if you need it. 


\section{Appendix 4.2: Low uncertainty treatments Instructions (Part I)}

This experiment is a study of individual and group decision-making. If you follow these instructions carefully and make informed decisions, you will earn money. The money you earn will be paid to you, in cash, at the end of the experiment.

You will be in a group consisting of six players. Each player plays the role of a firm. Your firm and five other firms are located near a lake next to a town. Anyone in the town can access the lake to go fishing, swimming or boating.

Your firm makes money through its production. Production also generates emissions, which affect the water quality of the lake. In general, the higher your production, the more you earn from production, but the greater the emissions your firm generates. Firm earnings are denominated in "experimental dollars", which will be exchanged for cash at the end of the experiment at the rate of 1750 experimental dollars to $\$ 1$.

This experiment is broken up into three parts and $\mathbf{1 0 0}$ decision "rounds", 45 rounds in Part I, 45rounds in Part II and 10 rounds in Part III. Each round is independent, meaning that decisions during a round do not affect future rounds in any way. In each round your task is to choose among six management options, labeled " $A$ " through " $F$ ". These options remain constant throughout the experiment. Associated with each management option is: (1) a production level (Production); (2) the average emissions generated (Average Emissions); and (3) the number of experimental dollars you earn from product sales (Earnings from Production). Your final earnings at the end of the experiment is whatever you earn in the 100 rounds based on you and your group members' decisions, plus a show-up fee of $\$ 5$.

You have been provided the following table (Table 1) titled "Initial Earnings" that lists the emissions, levels of production, and earnings from production that are associated with each management option. Please refer to this table before making any decisions.

\section{Table 1: Initial Earnings}

\begin{tabular}{cccc}
\hline Management Option & Average Emissions & Production & $\begin{array}{c}\text { Earnings from production } \\
\text { (Experimental Dollars) }\end{array}$ \\
\hline A & 6 & 150 & 800 \\
B & 5 & 138 & 797 \\
C & 4 & 123 & 786 \\
D & 3 & 108 & 764 \\
E & 2 & 89 & 725 \\
F & 1 & 66 & 655 \\
\hline
\end{tabular}

In addition to the management option, you and other firms choose, a variety of uncontrollable factors affect emissions of your firm. For example, heavy rainfall can 
increase the amount of water that flows over the land surface, increasing emissions. You cannot control those factors. However, the average resulting emissions of each management option can be estimated based on scientific data. For example, if you choose management option A, emissions of your firm could be below 6 or above 6 , but on average emissions would be 6 . In other words, if you choose option A many times and calculate the average of the resulting emissions, the average will be about 6 . Table 2 gives details of management options and associated probabilities of emissions.

The probabilities listed in Table 2 list the chance that a certain level of emissions are observed. To understand these probabilities, consider a lottery machine that has 100 balls numbered 1 through 100. If randomly drawn, any one ball has a $1 \%$ chance of being drawn because there are 100 balls. Larger probabilities can be considered by thinking about groups of balls. For instance, there is a 50\% probability of a ball being drawn at random that has a number on it greater than 50 because half the 100 balls have a number greater than 50. In other words, if you choose balls from the lottery many times, about half of the balls you choose will be with numbers greater than 50 .

Table 2: Probability of emissions levels and associated management options

\begin{tabular}{|c|c|c|c|c|c|c|c|c|}
\hline \multirow[b]{2}{*}{$\begin{array}{l}\text { Manageme } \\
\text { nt Option }\end{array}$} & \multirow[b]{2}{*}{$\begin{array}{l}\text { Average } \\
\text { emissio } \\
\text { ns }\end{array}$} & \multicolumn{7}{|c|}{ Probabilities } \\
\hline & & $\begin{array}{l}\text { Emissio } \\
\text { ns 0-1 }\end{array}$ & $\begin{array}{l}\text { Emissio } \\
\text { ns 1-2 }\end{array}$ & $\begin{array}{l}\text { Emissio } \\
\text { ns 2-3 }\end{array}$ & $\begin{array}{l}\text { Emissio } \\
\text { ns 3-4 }\end{array}$ & $\begin{array}{l}\text { Emissio } \\
\text { ns 4-5 }\end{array}$ & $\begin{array}{l}\text { Emissio } \\
\text { ns 5-6 }\end{array}$ & $\begin{array}{l}\text { Emissio } \\
\mathrm{ns}>6\end{array}$ \\
\hline $\mathrm{A}$ & 6 & $0.04 \%$ & $2.53 \%$ & $\begin{array}{l}10.22 \\
\%\end{array}$ & $\begin{array}{l}15.95 \\
\%\end{array}$ & $\begin{array}{l}16.69 \\
\%\end{array}$ & $\begin{array}{l}14.44 \\
\%\end{array}$ & $\begin{array}{l}40.13 \\
\%\end{array}$ \\
\hline B & 5 & $0.15 \%$ & $5.53 \%$ & $\begin{array}{l}16.34 \\
\%\end{array}$ & $\begin{array}{l}20.20 \\
\%\end{array}$ & $\begin{array}{l}17.65 \\
\%\end{array}$ & $\begin{array}{l}13.19 \\
\%\end{array}$ & $\begin{array}{l}26.94 \\
\%\end{array}$ \\
\hline $\mathrm{C}$ & 4 & $0.58 \%$ & $\begin{array}{l}12.21 \\
\%\end{array}$ & $\begin{array}{l}24.45 \\
\%\end{array}$ & $\begin{array}{l}22.63 \\
\%\end{array}$ & $\begin{array}{l}15.82 \\
\%\end{array}$ & $9.88 \%$ & $\begin{array}{l}14.44 \\
\%\end{array}$ \\
\hline $\mathrm{D}$ & 3 & $2.58 \%$ & $\begin{array}{l}26.17 \\
\%\end{array}$ & $\begin{array}{l}31.13 \\
\%\end{array}$ & $\begin{array}{l}19.67 \\
\%\end{array}$ & $\begin{array}{l}10.28 \\
\%\end{array}$ & $5.09 \%$ & $5.09 \%$ \\
\hline $\mathrm{E}$ & 2 & $\begin{array}{l}12.79 \\
\%\end{array}$ & $\begin{array}{l}47.08 \\
\%\end{array}$ & $\begin{array}{l}25.69 \\
\%\end{array}$ & $9.35 \%$ & $3.22 \%$ & $1.14 \%$ & $0.72 \%$ \\
\hline $\mathrm{F}$ & 1 & $\begin{array}{l}59.87 \\
\%\end{array}$ & $\begin{array}{l}35.04 \\
\%\end{array}$ & $4.37 \%$ & $0.59 \%$ & $0.10 \%$ & $0.02 \%$ & $0.01 \%$ \\
\hline
\end{tabular}

To fully understand Table 2, we further explain some examples.

Take a look at the first row. If you choose management option A in many rounds, the emissions of your firm will be 6 on average indicated by the number in the first cell. However, in any specific round, your firm's emissions might be different from 6 .

The second cell tells us that there is $0.04 \%$ chance that the emission level is equal or less than 1. It means if you choose management option A in 10,000 rounds, 
approximately 4 times your firm's emissions are less than or equal to 1 . In other words, the chance having an emission level between 0 and 1 if you choose option $A$ is same as the chance picking the balls numbered from 1 through 4 from a 10, 000 ball numbered from 1 through 10,000.

The next cell shows that the chance for your firm of emitting between 1 and 2 is $2.53 \%$. It means the chance of having an emission level between 1 and 2 if you choose option $\mathrm{A}$ is the same as the chance of picking the balls numbered from 1 through 253 from a 10, 000 ball numbered from 1 through 10,000. In other words, if you choose management option A in 10,000 rounds, for about 253 times your firm's emissions are greater than 1 and less than 2.

Finally, the chance for your firm of emitting greater than 6 is $40.13 \%$, noted by the last cell. It means the chance of having an emission level greater than 6 if you choose option A is the same as the chance of picking the balls numbered from 1 through 4,013 from a 10, 000 ball numbered from 1 through 10,000. In other words, if you choose management option A in 10,000 rounds, for about 4,013 times your firm's emissions is greater than 6.

In general, your firm's emissions are more likely to be lower if you choose a management option with low average emissions. Likewise, your firm's emissions are more likely to be higher if you choose a management option with high average emissions.

Your firm's emissions will result in pollution in the lake. Pollution affects the wellbeing of all lake users. For example, high pollution levels affect the health of fish, causing losses to fisherman. In order to protect the water resource, an environmental regulator requires you, and all other firms in your group, to pay the following Tax on pollution:

\section{Tax $=30 *$ Total Pollution}

Total Pollution equals the total of your firm's emissions and the other five players' emissions. In other words, the total pollution is based on the management decision of everyone in your group, not just your own.

A round of the experiment is complete when all six players have made their management decision by choosing from the six management options listed on your computer screen. After every player makes his or her management decision, Total Pollution will be calculated by the computer. The Tax will be calculated using the formula above. For each round, your Total Earnings is calculated as follows:

\section{Total Earnings = Earnings from Production - Tax}

Your computer will show your Management Options, your Earnings from Production, the Total Pollution, the Tax and your Total Earnings from the current 
round and all previous rounds. Your computer will also show you other five players' Management Options after everyone makes their decision in one round.

Here is one example of how to calculate your Total Earnings. If in one round each of five others in your group chooses option $\mathrm{E}$ and has an individual emission of 2 and you choose option A and have an individual emission of 6, the Total Pollution will be 16. Everyone in your group will pay a tax that is equal to 480 experimental dollars. Your total earnings in this round will be $800-480=320$ experimental dollars.

Here is another example. If in one round all of you choose option A and each of you have an individual emission of 6, the total pollution will be 36. Everyone in your group will pay a tax that is equal to 1080 experimental dollars. Your total earnings in this round will be $800-1080=-220$ experimental dollars, which means you lose 220 experimental dollars.

It is important that you understand the instructions and the experiment. We will ask you a few questions before you make any decisions in the experiment. The computer will check your answers automatically. If you do not get the correct answers after a few attempts, please let the experiment administrator know and he or she will be happy to explain them to you. 


\section{Please do not turn to next page unless experimenters ask you to do so.}




\section{Instructions (Part II)}

You will make another 45 decisions in this part. Everything in the game will keep the same except that other firms' management option choices in your group will not be given to you anymore. Please also read the instructions in Part I to make any decisions if you need it. 


\section{Appendix 4.3: High uncertainty treatments Instructions (Part I)}

This experiment is a study of individual and group decision-making. If you follow these instructions carefully and make informed decisions, you will earn money. The money you earn will be paid to you, in cash, at the end of the experiment.

You will be in a group consisting of six players. Each player plays the role of a firm. Your firm and five other firms are located near a lake next to a town. Anyone in the town can access the lake to go fishing, swimming or boating.

Your firm makes money through its production. Production also generates emissions, which affect the water quality of the lake. In general, the higher your production the more you earn from production, but the greater the emissions your firm generates. Firm earnings are denominated in "experimental dollars", which will be exchanged for cash at the end of the experiment at the rate of 1750 experimental dollars to $\$ 1$.

This experiment is broken up into three parts and $\mathbf{1 0 0}$ decision "rounds", 45 rounds in Part I, 45rounds in Part II and 10 rounds in Part III. Each round is independent, meaning that decisions during a round do not affect future rounds in any way. In each round your task is to choose among six management options, labeled " $A$ " through " $F$ ". These options remain constant throughout the experiment. Associated with each management option is: (1) a production level (Production); (2) the average emissions generated (Average Emissions); and (3) the number of experimental dollars you earn from product sales (Earnings from Production). Your final earnings at the end of the experiment is whatever you earn in the 100 rounds based on you and your group members' decisions, plus a show-up fee of $\$ 5$.

You have been provided the following table (Table 1) titled "Initial Earnings" that lists the emissions, levels of production, and earnings from production that are associated with each management option. Please refer to this table before making any decisions.

\section{Table 1: Initial Earnings}

\begin{tabular}{llll}
\hline Management Option & Average Emissions & Production & $\begin{array}{l}\text { Earnings from production } \\
\text { (Experimental Dollars) }\end{array}$ \\
\hline A & 6 & 150 & 800 \\
B & 5 & 138 & 797 \\
C & 4 & 123 & 786 \\
D & 3 & 108 & 764 \\
E & 2 & 89 & 725 \\
F & 1 & 66 & 655 \\
\hline
\end{tabular}

In addition to the management option, you and other firms choose, a variety of uncontrollable factors affect emissions of your firm. For example, heavy rainfall can increase the amount of water that flows over the land surface, increasing emissions. You 
cannot control those factors. However, the average resulting emissions of each management option can be estimated based on scientific data. For example, if you choose management option A, emissions of your firm could be below 6 or above 6 , but on average emissions would be 6 . In other words, if you choose option A many times and calculate the average of the resulting emissions, the average will be about 6 . Table 2 gives details of management options and associated probabilities of emissions.

The probabilities listed in Table 2 list the chance that a certain level of emissions are observed. To understand these probabilities, consider a lottery machine that has 100 balls numbered 1 through 100 . If randomly drawn, any one ball has a $1 \%$ chance of being drawn because there are 100 balls. Larger probabilities can be considered by thinking about groups of balls. For instance, there is a $50 \%$ probability of a ball being drawn at random that has a number on it greater than 50 because half the 100 balls have a number greater than 50 . In other words, if you choose balls from the lottery many times, about half of the balls you choose will be with numbers greater than 50 .

\section{Table 2: Probability of emissions levels and associated management options}

\begin{tabular}{|c|c|c|c|c|c|c|c|c|}
\hline \multirow[b]{2}{*}{$\begin{array}{l}\text { Manageme } \\
\text { nt Option }\end{array}$} & \multirow[b]{2}{*}{$\begin{array}{l}\text { Average } \\
\text { emissio } \\
\text { ns }\end{array}$} & \multicolumn{7}{|c|}{ Probabilities } \\
\hline & & $\begin{array}{l}\text { Emissio } \\
\text { ns 0-1 }\end{array}$ & $\begin{array}{l}\text { Emissio } \\
\text { ns 1-2 }\end{array}$ & $\begin{array}{l}\text { Emissio } \\
\text { ns } 2-3\end{array}$ & $\begin{array}{l}\text { Emissio } \\
\text { ns 3-4 }\end{array}$ & $\begin{array}{l}\text { Emissio } \\
\text { ns 4-5 }\end{array}$ & $\begin{array}{l}\text { Emissio } \\
\text { ns 5-6 }\end{array}$ & $\begin{array}{l}\text { Emissio } \\
\mathrm{ns}>6\end{array}$ \\
\hline $\mathrm{A}$ & 6 & $9.82 \%$ & $17.65 \%$ & $14.87 \%$ & $11.42 \%$ & $8.70 \%$ & $6.68 \%$ & $30.85 \%$ \\
\hline B & 5 & $13.36 \%$ & $20.50 \%$ & $15.71 \%$ & $11.34 \%$ & $8.24 \%$ & $6.10 \%$ & $24.75 \%$ \\
\hline $\mathrm{C}$ & 4 & $18.77 \%$ & $23.57 \%$ & $16.06 \%$ & $10.74 \%$ & $7.37 \%$ & $5.22 \%$ & $18.26 \%$ \\
\hline D & 3 & $27.47 \%$ & $26.29 \%$ & $15.38 \%$ & $9.31 \%$ & $5.94 \%$ & $3.96 \%$ & $11.64 \%$ \\
\hline E & 2 & $42.34 \%$ & $26.80 \%$ & $12.59 \%$ & $6.62 \%$ & $3.81 \%$ & $2.34 \%$ & $5.50 \%$ \\
\hline $\mathrm{F}$ & 1 & $69.15 \%$ & $19.21 \%$ & $6.15 \%$ & $2.53 \%$ & $1.22 \%$ & $0.65 \%$ & $1.10 \%$ \\
\hline
\end{tabular}

To fully understand Table 2, we further explain some examples.

Take a look at the first row. If you choose management option $\mathrm{A}$ in many rounds, the emissions of your firm will be 6 on average indicated by the number in the first cell. However, in any specific round, your firm's emissions might be different from 6 .

The second cell tells us that there is $9.82 \%$ chance that the emission level is equal or less than 1. It means if you choose management option A in 10,000 rounds, approximately 982 times your firm's emissions are less than or equal to 1 . In other words, the chance having an emission level between 0 and 1 if you choose option $\mathrm{A}$ is same as the chance picking the balls numbered from 1 through 982 from a 10, 000 ball numbered from 1 through 10,000.

The next cell shows that the chance for your firm of emitting between 1 and 2 is $17.65 \%$. It means the chance of having an emission level between 1 and 2 if you choose option $\mathrm{A}$ is the same as the chance of picking the balls numbered from 1 through 1,765 from a 
10, 000 ball numbered from 1 through 10,000. In other words, if you choose management option A in 10,000 rounds, for about 1,765 times your firm's emissions are greater than 1 and less than 2 .

Finally, the chance for your firm of emitting greater than 6 is $30.85 \%$, noted by the last cell. It means the chance of having an emission level greater than 6 if you choose option A is the same as the chance of picking the balls numbered from 1 through 3,085 from a 10, 000 ball numbered from 1 through 10,000. In other words, if you choose management option A in 10,000 rounds, for about 3,085 times your firm's emissions is greater than 6.

In general, your firm's emissions are more likely to be lower if you choose a management option with low average emissions. Likewise, your firm's emissions are more likely to be higher if you choose a management option with high average emissions.

Your firm's emissions will result in pollution in the lake. Pollution affects the wellbeing of all lake users. For example, high pollution levels affect the health of fish, causing losses to the fisherman. To protect the water resource, an environmental regulator requires you, and all other firms in your group, to pay the following Tax on pollution:

\section{Tax $=30 *$ Total Pollution}

Total Pollution equals the total of your firm's emissions and the other five players' emissions. In other words, the total pollution is based on the management decision of everyone in your group, not just your own.

A round of the experiment is complete when all six players have made their management decision by choosing from the six management options listed on your computer screen. After every player makes his or her management decision, Total Pollution will be calculated by the computer. The Tax will be calculated using the formula above. For each round, your Total Earnings is calculated as follows:

\section{Total Earnings $=$ Earnings from Production - Tax}

Your computer will show your Management Options, your Earnings from Production, the Total Pollution, the Tax and your Total Earnings from the current round and all previous rounds. Your computer will also show you other five players' Management Options after everyone makes their decision in one round.

Here is an example of how to calculate your Total Earnings. If in one round each of five others in your group chooses option $\mathrm{E}$ and has an individual emission of 2 and you choose option A and have an individual emission of 6, the Total Pollution will be 16. Everyone in your group will pay a tax that is equal to 480 experimental dollars. Your total earnings in this round will be $800-480=320$ experimental dollars. 
Here is another example. If in one round all of you choose option A and each of you have an individual emission of 6, the total pollution will be 36. Everyone in your group will pay a tax that is equal to 1080 experimental dollars. Your total earnings in this round will be $800-1080=-220$ experimental dollars, which means you lose 220 experimental dollars.

It is important that you understand the instructions and the experiment. We will ask you a few questions before you make any decisions in the experiment. The computer will check your answers automatically. If you do not get the correct answers after a few attempts, please let the experiment administrator know and he or she will be happy to explain them to you. 


\section{Please do not turn to next page unless experimenters ask you to do so.}




\section{Instructions (Part II)}

You will make another 45 decisions in this part. Everything in the game will keep the same except that other firms' management option choices in your group will not be given to you anymore. Please also read the instructions in Part I to make any decisions if you need it. 